TI 2012-009/4

Tinbergen Institute Discussion Paper
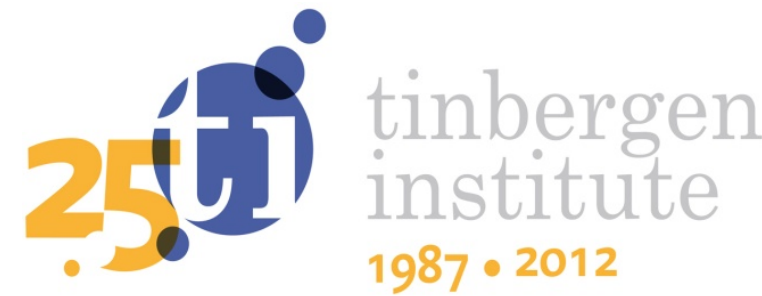

\title{
Generalized Dynamic Panel Data Models with Random Effects for Cross-Section and Time
}

Geert Mestersa,b

Siem Jan Koopmanb,c

a Netherlands Institute for the Study of Crime and Law Enforcement;

b Department of Econometrics, VU University Amsterdam;

c Tinbergen Institute. 
Tinbergen Institute is the graduate school and research institute in economics of Erasmus University Rotterdam, the University of Amsterdam and VU University Amsterdam.

More TI discussion papers can be downloaded at http://www.tinbergen.nl

Tinbergen Institute has two locations:

Tinbergen Institute Amsterdam

Gustav Mahlerplein 117

1082 MS Amsterdam

The Netherlands

Tel.: +31(0)205251600

Tinbergen Institute Rotterdam

Burg. Oudlaan 50

3062 PA Rotterdam

The Netherlands

Tel.: +31(0)10 4088900

Fax: $+31(0) 104089031$

Duisenberg school of finance is a collaboration of the Dutch financial sector and universities, with the ambition to support innovative research and offer top quality academic education in core areas of finance.

DSF research papers can be downloaded at: http://www.dsf.nl/

Duisenberg school of finance

Gustav Mahlerplein 117

1082 MS Amsterdam

The Netherlands

Tel.: +31(0)20 5258579 


\title{
Generalized Dynamic Panel Data Models with Random Effects for Cross-Section and Time
}

\author{
G. Mesters ${ }^{(a, b, c) *}$ and S.J. Koopman ${ }^{(b, c)}$ \\ (a) Netherlands Institute for the Study of Crime and Law Enforcement, \\ (b) Department of Econometrics, VU University Amsterdam, \\ (c) Tinbergen Institute, Amsterdam
}

January 25, 2014

\begin{abstract}
An exact maximum likelihood method is developed for the estimation of parameters in a nonlinear non-Gaussian dynamic panel data model with unobserved random individualspecific and time-varying effects. We propose an estimation procedure based on the importance sampling technique. In particular, a sequence of conditional importance densities is derived which integrates out all random effects from the joint distribution of endogenous variables. We disentangle the integration over both the cross-section and the time series dimensions. The estimation method facilitates the modeling of large panels in both dimensions. We evaluate the method in an extended Monte Carlo study for dynamic panel data models with observations from different non-Gaussian distributions. We finally present three empirical illustrations for (i) union choice of young males using a Binary panel, (ii) crime rates of families using a Binomial panel and (iii) economic growth modeling using a Student's $t$ panel.
\end{abstract}

JEL classification: C33; C51

Some keywords: Dynamic panel data; non-Gaussian; Importance Sampling; Random Effects.

${ }^{*}$ Corresponding author: G. Mesters, Department of Econometrics, VU University Amsterdam, The Netherlands. Contact address: NSCR, PO box 71304, 1008 BH Amsterdam, The Netherlands, tel. nr. +31 2059 89405, email: gmesters@nscr.nl, Technical Appendix: http://personal.vu.nl/s.j.koopman and http://www.geertmesters.nl. We would like to thank the Editor, the Associate Editor and two referees for their supportive comments on earlier versions of this paper. 


\section{Introduction}

In this paper we develop a Monte Carlo maximum likelihood procedure for the estimation of parameters in a generalized dynamic panel data model. The model consists of a nonlinear non-Gaussian density for the observations conditional on a latent signal. We decompose the latent signal into a fixed component and a stochastic component. The fixed component is defined as a linear function of explanatory variables and lagged observations, whereas the stochastic component includes random individual-specific effects and time-varying effects. The two effects are assumed to come from mutually independent Gaussian densities. When the density of the observations is considered to be conditionally Gaussian with mean equal to the latent signal and some arbitrary variance, the model reduces to the linear Gaussian random effects panel data model as studied in Hsiao (2003) and Baltagi (2005).

Maximum likelihood estimation is not trivial for the generalized dynamic panel data model because the likelihood does not exist in closed form. The functional form of the observation density together with the stochastic component of the latent signal prohibit closed form solutions. For a simultaneous analysis of random individual-specific and timevarying effects we extend the methods of Shephard \& Pitt (1997) and Durbin \& Koopman (1997) which are based on Monte Carlo simulation methods. In particular, they adopt an importance sampler for which an approximating linear Gaussian state space model is used to draw samples of latent signals. We extend their method for the treatment of random individual-specific effects. We construct a sequence of conditional importance densities that sequentially integrates out random effects from the joint distribution. We disentangle the integration over the cross-section dimension (for the individual-specific effects) and the time series dimension (for the time-varying effects). The constructed importance densities are based on a linear Gaussian dynamic panel data model which sufficiently approximates the true model for the simulation of latent signals.

We further show that the panel of time series can be collapsed into two low-dimensional vector series, which are used to sample the random individual-specific and time-varying 
effects from the importance densities. In particular, we collapse the cross-sectional dimension of $y$ without compromising any information that is needed to sample the time-varying effects and visa versa. The transformations are based on modifications of the methods introduced in Jungbacker \& Koopman (2008) and lead to large computational savings when evaluating the Monte Carlo likelihood.

For linear dynamic panel data models, transformations can be adopted to eliminate the individual-specific effects, whereas time-varying effects are typically modeled using deterministic functions. Then instrumental variables can be found for the implementation in a generalized method of moments framework, see for example Arellano \& Bond (1991) and Blundell \& Bond (1998). For most non-Gaussian dynamic panel data models no known transformations exist to eliminate the individual-specific effects. Therefore, it is common to assume stochastic specifications for these effects, see the discussion in Wooldridge (2005). In order to integrate the random effects from the complete likelihood, several other Monte Carlo estimation methods have been proposed in the literature. Examples for models without time-varying effects, are simulated maximum likelihood approaches based on the GewekeHajivassiliou-Keane (GHK) sampler, see Geweke (1991), Hajivassiliou (1990) and Keane (1994), and the more general Markov Chain Monte Carlo methods including Gibbs sampling and the Metropolis-Hastings algorithm, see Geweke \& Keane (2001) and Chib (2008). Arellano \& Bonhomme (2011) discuss further advances in nonlinear panel data analysis for models without time-varying random effects.

Richard \& Zhang (2007) and Liesenfeld \& Richard (2008) show that simulation based inference is possible using their efficient importance sampling (EIS) method for non-Gaussian dynamic panel data models with individual-specific and time-varying effects. Our method differs from the Richard \& Zhang (2007) approach in several ways. First, we disentangle the Monte Carlo integration over the individual-specific and time-varying effects by conditioning on the posterior modal values of the time-varying and individual-specific effects, respectively. This allows us to separate the treatment of the different random effects. Second, by transforming the panel into two low-dimensional vector series prior to sampling 
from the importance densities increasing panel dimensions only marginally impact the overall computational efficiency of our proposed estimation method. Third, the construction of the importance densities differs highly from each other.

Our proposed estimation method for the general model provides a number of additional benefits. First, when only individual-specific effects are included in our model, our sampler remains highly accurate despite the length of the time series dimension. In this respect we improve on the GHK sampler based simulation method, whose performance is shown to deteriorate as the time series dimension becomes large, see Lee (1997). Similar improvements, using the EIS methodology for probit panel data models, have been proposed by Liesenfeld, Moura \& Richard (2010) and Liesenfeld \& Richard (2010). Second, our framework allows for the simultaneous analysis of unobserved heterogeneity, state dependence and correlated error terms. This is useful as in many empirical panel data studies there are multiple underlying sources of error. Third, parameter heterogeneity can be imposed for our nonGaussian framework with respect to the time periods as well as the individuals by following the implementations described in Harvey (1989) and Hsiao \& Pesaran (2008). They discuss heterogeneous parameters in the context of linear models. Fourth, the estimation method can be computationally modified to handle missing values and unbalanced panels. Additional methods are not necessary and it contrasts with the two-step procedures as developed by, for example, Stock \& Watson (2002).

The remainder of the paper is organized as follows. Section 2 formally describes the generalized dynamic panel data model in detail. In Section 3 we develop our Monte Carlo maximum likelihood method for the general model. Section 4 evaluates the performance of our estimation method in a simulation study. We consider dynamic panel data models with Binary, Binomial and Student's $t$ densities. In Section 5 we present empirical studies for the union choice of young males, the crime rates of families and economic growth rates of countries. The empirical studies highlight the flexibility of our framework with respect to handling missing values, state dependence and parameter heterogeneity. Section 6 summarizes our findings. 


\section{Statistical model formulation}

We formally define the generalized dynamic panel data model for observations of variable $y_{i, t}$, that is associated with individual $i$ and time $t$. Data is available for $N$ individuals. For each individual $i$ the time series dimension is $T_{i}$, for $i=1, \ldots, N$. Each time period is indexed by $t$. The entire time span of the unbalanced panel is restricted between some arbitrary starting period $t=1$ and the final period $t=T$. The general model for variable $y_{i, t}$ is given by

$$
y_{i, t} \stackrel{i . i . d .}{\sim} p\left(y_{i, t} \mid z_{i, t} ; \psi\right)
$$

where $z_{i, t}$ is the signal for $y_{i, t}$ and $p\left(y_{i, t} \mid z_{i, t} ; \psi\right)$ is a density that depends on the parameter vector $\psi$. We assume that $p\left(y_{i, t} \mid z_{i, t} ; \psi\right)$ is possibly non-Gaussian and is correctly specified. In Section 5 we consider the Binary, Binomial and Student's $t$ densities as examples for $p\left(y_{i, t} \mid z_{i, t} ; \psi\right)$ and illustrate their empirical relevance. The latent signal $z_{i, t}$ incorporates all dynamics, covariates and stochastic processes driving the density $p\left(y_{i, t} \mid z_{i, t} ; \psi\right)$. A general decomposition of signal $z_{i, t}$ is given by

$$
z_{i, t}=w_{i, t}+\epsilon_{i, t}
$$

where $w_{i, t}$ is a fixed component and $\epsilon_{i, t}$ is a stochastic component. The components are given by

$$
w_{i, t}=x_{i, t}^{\prime} \beta+\gamma_{1} y_{i, t-1}+\ldots+\gamma_{p_{y}} y_{t-p_{y}}, \quad \text { and } \quad \epsilon_{i, t}=a_{i, t}^{\prime} \mu_{i}+b_{i, t}^{\prime} \xi_{t},
$$

where $x_{i, t}$ is a $k \times 1$ vector of observable explanatory variables, $\beta$ is a $k \times 1$ parameter vector, $\gamma_{j}$ is an unknown parameter for $j=1, \ldots, p_{y}$ and for some non-negative integer $p_{y}$, the $q \times 1$ vector of individual-specific effects $\mu_{i}$ for individual $i$ is weighted at time period $t$ by the $q \times 1$ vector $a_{i, t}$ and the $r \times 1$ vector of time-varying effects $\xi_{t}$ is weighted for individual $i$ at time period $t$ by $r \times 1$ vector $b_{i, t}$. Both weight vectors, $a_{i, t}$ and $b_{i, t}$, are assumed fixed and may depend on the parameter vector $\psi$. 
The individual-specific effects $\mu_{i}$ and the time-varying effects $\xi_{t}$ are given by

$$
\mu_{i} \sim \operatorname{NID}\left(\delta, \Sigma_{\mu}\right), \quad \xi_{t}=G \alpha_{t}, \quad \alpha_{t+1}=H \alpha_{t}+R \eta_{t}, \quad \eta_{t} \sim \operatorname{NID}\left(0, \Sigma_{\eta}\right)
$$

where the individual effects $\mu_{i}$ are assumed normally and independently distributed, with $q \times 1$ common mean vector $\delta$ and $q \times q$ variance matrix $\Sigma_{\mu}$, which are both considered fixed, the $r \times p$ dimensional matrix $G$ relates the generating linear autoregressive process $\alpha_{t}$ to the time-varying effects $\xi_{t}, H$ is a $p \times p$ transition matrix, $R$ is a $p \times l$ disturbance selection matrix, and $\eta_{t}$ is an $l \times 1$ vector of disturbances with variance matrix $\Sigma_{\eta}$. The system matrices $G, H, R$ and $\Sigma_{\eta}$ are fixed and known, although some elements may depend on parameter vector $\psi$. The initial state vector $\alpha_{1}$ is assumed normally distributed with mean zero and variance matrix $P$. Individual-specific effects $\mu_{i}$ and $\mu_{j}$ are assumed mutually uncorrelated and independent from the time-varying effects, $\xi_{t}$, for all $i, j=1, \ldots, N$ and $t=1, \ldots, T$.

For the initial signal $z_{i, 1}$ we assume that $y_{i, t}$, for $t<1$, are fixed and known constants for all $i=1, \ldots, N$. Also, we will assume that parameters $\beta$ and $\gamma$ are common for all individuals and time periods, and that explanatory variables $x_{i, t}$ are exogenous and uncorrelated with $\epsilon_{j, t}$, for all $i, j=i, \ldots, N$ and common time periods $t$. Section 5 discusses options for relaxing these assumptions. In particular, there we adopt the methods developed in Chamberlain (1980), Wooldridge (2005), Hsiao \& Pesaran (2008) and Harvey (1989).

The specification of the signal allows us to distinguish between two sources capable of explaining the dynamics between particular outcomes, see Heckman $(1981 a, b)$. The first source is the presence of "true state dependence", which is the phenomenon that past outcomes provide explanatory power for future outcomes. This is represented in our model by the terms $\gamma_{1} y_{i, t-1}+\ldots+\gamma_{p_{y}} y_{i, t-p_{y}}$. The second source, referred to by Heckman (1981a) as "spurious state dependence", explains dynamics as resulting from serial correlation in stochastic component $\epsilon_{i, t}$. We aim to capture serial correlation in $\epsilon_{i, t}$ by including individualspecific effects $\mu_{i}$ and time-varying effects $\xi_{t}$.

The general model contains many parameters. To identify these parameters in the model 
different strategies can be considered of which the appropriateness needs to be evaluated on a case by case basis. In general we need to restrict either the distribution of $\mu_{i}, \xi_{t}$ or a combination of both. Further, as only a limited number of elements of weight vectors $a_{i, t}$ and $b_{i, t}$ can be identified, some hierarchical constraints must be imposed.

The generalized dynamic panel data model of this paper is fully defined by equations (1), (2), (3), and (4). All parameters are collected in vector $\psi$ and typically contain parameters affecting signal $z_{i, t}$. Under the assumption that the model is correctly specified, the density of the observations $y=\left\{y_{i, t}\right\}$ conditional on signal $z=\left\{z_{i, t}\right\}$ is given by

$$
p(y \mid z ; \psi)=\prod_{i=1}^{N} \prod_{t=1}^{T_{i}} p\left(y_{i, t} \mid z_{i, t} ; \psi\right)=\prod_{i=1}^{N} p\left(y_{i, 1} \mid z_{i, 1} ; \psi\right) \prod_{t=2}^{T_{i}} p\left(y_{i, t} \mid \mu_{i}, \xi_{t} ; x_{i, t}, \mathcal{Y}_{i, t-1}, \psi\right)
$$

where $\mathcal{Y}_{i, t}=\left\{y_{i, 1}, \ldots, y_{i, t}\right\}$. The last equality is partly the result of the prediction error decomposition.

\section{Likelihood evaluation by Monte Carlo integration}

This section discusses the method of Monte Carlo maximum likelihood for the estimation of the parameter vector $\psi$. We first consider the generalized dynamic panel data model for balanced panels, $T_{i}=T$ for all $i=1, \ldots, N$. In Section 3.4 we provide the necessary alterations for the treatment of unbalanced panels. The loglikelihood for observation vector $y$ is defined by $\ell(\psi)=\log p(y ; \psi)$, where $p(y ; \psi)$ denotes the joint density of all observations for parameter vector $\psi$. In the remainder of this section we drop the dependence on parameter vector $\psi$ for notational convenience. For example, we have $\log p(y) \equiv \log p(y ; \psi)$.

In the presence of unobserved random individual-specific and time-varying effects, $\mu=$ $\left\{\mu_{i}\right\}$ and $\xi=\left\{\xi_{t}\right\}$, density $p(y)$ can be expressed as

$$
p(y)=\int_{z} p(y, z) \mathrm{d} z=\int_{\xi} \int_{\mu} p(y, \mu, \xi ; x) \mathrm{d} \mu \mathrm{d} \xi=\int_{\xi} \int_{\mu} p(y \mid \mu, \xi ; x) p(\mu, \xi) \mathrm{d} \mu \mathrm{d} \xi
$$

where the second equality holds as $x=\left\{x_{i, t}\right\}$ is deterministic and where $p(\mu, \xi)=p(\mu) p(\xi)$, 
since $\mu$ and $\xi$ are independent. The evaluation of the high dimensional integral $(6)$ is complicated because an analytical solution is not available for the nonlinear non-Gaussian density $p(y \mid \mu, \xi ; x)=p(y \mid z)$. We propose to solve the integral by Monte Carlo integration using the importance sampling technique, see Ripley (1987).

A general importance sampling representation for $p(y)$ is given by

$$
p(y)=\int_{\xi} \int_{\mu} \frac{p(y \mid \mu, \xi ; x) p(\mu, \xi)}{g(\mu, \xi \mid y)} g(\mu, \xi \mid y) \mathrm{d} \mu \mathrm{d} \xi
$$

where $g(\mu, \xi \mid y)$ denotes the importance density. Integral (7) can be solved by Monte Carlo integration for which we sample $\mu^{(i)}$ and $\xi^{(i)}$ from the importance density $g(\mu, \xi \mid y)$ and compute estimate $M^{-1} \sum_{i=1}^{M} p\left(y, \mu^{(i)}, \xi^{(i)} ; x\right) / g\left(\mu^{(i)}, \xi^{(i)} \mid y\right)$.

For any choice of the density $g(\mu, \xi \mid y)$, which accounts for the complicated a-posteriori covariance structure of $\mu$ and $\xi$, sampling from it is likely to be complicated. More specific, as $y$ depends on both $\mu$ and $\xi$, there exists correlation between all individuals (due to $\xi$ ) and time periods (due to $\mu$ ). This makes sampling from densities $g(\mu, \xi \mid y)$ that account for these correlations infeasible even for moderate panel sizes. As a consequence, the importance sampling based methods of Durbin \& Koopman (1997) and Shephard \& Pitt (1997), which construct $g(\mu, \xi \mid y)$ based on an approximating linear Gaussian model, need to be modified in order to obtain a feasible importance sampler.

We propose to integrate out $\mu$ by keeping $\xi$ fixed at its posterior modal value and we propose to integrate out $\xi$ by keeping $\mu$ fixed at its posterior modal value. When either $\mu$ or $\xi$ is fixed the covariance structure of $y$ greatly simplifies. The posterior modal values are chosen for computational convenience. For density $p(y)$ from the generalized dynamic panel data model we propose the following importance sampling representation

$$
p(y)=\int_{\xi} \int_{\mu} \frac{p(y \mid \mu, \xi ; x) p(\mu) p(\xi)}{g(\mu \mid y ; \hat{\xi}) g(\xi \mid y ; \hat{\mu})} g(\mu \mid y ; \hat{\xi}) g(\xi \mid y ; \hat{\mu}) \mathrm{d} \mu \mathrm{d} \xi
$$

where $g(\mu \mid y ; \hat{\xi})$ and $g(\xi \mid y ; \hat{\mu})$ are the importance densities. We define $\hat{\mu}$ and $\hat{\xi}$ as the posterior 
modal values of $p(\mu, \xi \mid y ; x)$, which can be found iteratively as we discuss in Section 3.1. When applying Bayes rule twice to the right hand side of equation (8) we obtain

$$
p(y)=g(y ; \hat{\xi}) g(y ; \hat{\mu}) \int_{\xi} \int_{\mu} \frac{p(y \mid \mu, \xi ; x)}{g(y \mid \mu ; \hat{\xi}) g(y \mid \xi ; \hat{\mu})} g(\mu \mid y ; \hat{\xi}) g(\xi \mid y ; \hat{\mu}) \mathrm{d} \mu \mathrm{d} \xi
$$

where we have retained the marginal properties of $\mu$ and $\xi$ by imposing $g(\xi)=p(\xi)$ and $g(\mu)=p(\mu)$. It holds that $g(y ; \hat{\xi})$ and $g(y ; \hat{\mu})$ are the conditional likelihoods, which are implicitly defined by the choice for the importance densities. Under the assumption that the modes $\hat{\mu}$ and $\hat{\xi}$ are well defined and can be computed, we define $\hat{p}(y)$ as the Monte Carlo estimate of (9) and given by

$$
\hat{p}(y)=g(y ; \hat{\xi}) g(y ; \hat{\mu}) \frac{1}{M} \sum_{i=1}^{M} \frac{p\left(y \mid \mu^{(i)}, \xi^{(i)} ; x\right)}{g\left(y \mid \mu^{(i)} ; \hat{\xi}\right) g\left(y \mid \xi^{(i)} ; \hat{\mu}\right)}
$$

where samples $\left\{\mu^{(1)}, \ldots, \mu^{(M)}\right\}$ are drawn independently from importance density $g(\mu \mid y ; \hat{\xi})$ and samples $\left\{\xi^{(1)}, \ldots, \xi^{(M)}\right\}$ from $g(\xi \mid y ; \hat{\mu})$. The density $p\left(y \mid \mu^{(i)}, \xi^{(i)} ; x\right)$ is evaluated using equation (5).

The quality of the estimate in equation $(10)$ depends on how well the product of $g(\mu \mid y ; \hat{\xi})$ and $g(\xi \mid y ; \hat{\mu})$ approximates $p(y, \mu, \xi ; x)$, which needs to be evaluated on a case by case basis. The Lindeberg-Levy central limit theorem implies a $\sqrt{M}$ convergence rate for $\hat{p}(y) \rightarrow p(y)$ as $M \rightarrow \infty$ if draws from the importance sampler are independent and if importance weights

$$
w^{(i)}=p\left(y \mid \mu^{(i)}, \xi^{(i)} ; x\right) /\left[g\left(y \mid \xi^{(i)} ; \hat{\mu}\right) g\left(y \mid \mu^{(i)} ; \hat{\xi}\right)\right]
$$

have finite mean and variance, as argued in Geweke (1989). The last condition can be examined empirically using extreme value theory based tests proposed in Monahan (2001) and Koopman, Shephard \& Creal (2009). In the simulation study of Section 4 we consider diagnostic test statistics for the existence of a variance in a sequence of importance weights drawn from Binary, Binomial and Student's $t$ dynamic panel data models. 


\subsection{Constructing the importance density}

Next we consider the construction of importance densities $g(\mu \mid y ; \hat{\xi})$ and $g(\xi \mid y ; \hat{\mu})$, proposed for evaluating estimate $\hat{p}(y)$, given in equation 10 . We choose both densities to follow Gaussian distributions and modify their means and variances such that their modes are equal to the modes of the original posterior density $p(\mu, \xi \mid y ; x)$. Similar strategies are followed for models without random individual-specific effects, see, for example, Shephard \& Pitt (1997) and Durbin \& Koopman (1997, 2000). So (2003) and Jungbacker \& Koopman (2007) argue that this strategy can be implemented by numerically maximizing $\log p(\mu, \xi \mid y ; x)=$ $\log p(y \mid \mu, \xi ; x)+\log p(\mu, \xi)-\log p(y ; x)$ with respect to $\mu$ and $\xi$.

The instrumental basis to facilitate this numerical maximization is given, for variable $y_{i, t}$, by the linear Gaussian panel data model

$$
y_{i, t}=c_{i, t}+\epsilon_{i, t}+u_{i, t}, \quad u_{i, t} \sim \operatorname{NID}\left(0, d_{i, t}^{2}\right)
$$

where $c_{i, t}$ is a fixed constant, stochastic component $\epsilon_{i, t}$ is given by equation (3) and $u_{i, t}$ is a random variable with mean zero and fixed variance $d_{i, t}^{2}$. The predetermined component $w_{i, t}$ is not explicitly included in approximating model 12 since it is fixed at time $t$. The constants $c_{i, t}$ and $d_{i, t}$ are chosen such that 12 can be used to compute the posterior modal

values $\hat{\mu}$ and $\hat{\xi}$, respectively. The elements $u_{i, t}$ and $\epsilon_{j, s}$ are uncorrelated with each other, for all $i, j=1, \ldots, N$ and $s, t=1, \ldots, T$. Furthermore, $u_{i, t}$ is serially uncorrelated. It follows that

$$
g(y \mid \mu, \xi)=\prod_{i=1}^{N} \prod_{t=1}^{T} g\left(y_{i, t} \mid \mu_{i}, \xi_{t}\right), \quad \text { with } \quad g\left(y_{i, t} \mid \mu_{i}, \xi_{t}\right) \equiv \operatorname{NID}\left(c_{i, t}+\epsilon_{i, t}, d_{i, t}^{2}\right)
$$

The maximization of $\log p(\mu, \xi \mid y ; x)$ with respect to $\mu$ and $\xi$ can be carried out via the Newton-Raphson method. The idea is to iterate between linearizing $p(y \mid \mu, \xi ; x)$, by computing $c=\left\{c_{i, t}\right\}$ and $d=\left\{d_{i, t}\right\}$, to obtain $g(y \mid \mu, \xi)$ and updating $\mu$ and $\xi$ based on the linearized model given by equations (12) and (3). The following algorithm summarizes this method. 


\section{Algorithm A}

(i) Initialize the algorithm by choosing $\mu^{*}$ and $\xi^{*}$ as starting values, which gives $\epsilon_{i, t}^{*}$ and $z_{i, t}^{*}$, for all $i=1, \ldots, N$ and $t=1, \ldots, T$

(ii) Given the set of two equations

$$
\frac{\partial \log p\left(y_{i, t} \mid z_{i, t}\right)}{\partial z_{i, t}}=\frac{\partial \log g\left(y_{i, t} \mid \epsilon_{i, t}\right)}{\partial \epsilon_{i, t}}, \quad \frac{\partial^{2} \log p\left(y_{i, t} \mid z_{i, t}\right)}{\partial z_{i, t} \partial z_{i, t}}=\frac{\partial^{2} \log g\left(y_{i, t} \mid \epsilon_{i, t}\right)}{\partial \epsilon_{i, t} \partial \epsilon_{i, t}}
$$

for $i=1, \ldots, N$ and $t=1, \ldots, T$, where $p\left(y_{i, t} \mid z_{i, t}\right)$ is the observation model (1) and $g\left(y_{i, t} \mid \epsilon_{i, t}\right)$ is given by (13), we can deduct expressions for $c_{i, t}$ and $d_{i, t}$ as functions of $z_{i, t}$, and compute $c_{i, t}=c_{i, t}^{*}$ and $d_{i, t}=d_{i, t}^{*}$ for $\epsilon_{i, t}=\epsilon_{i, t}^{*}$ and $z_{i, t}=z_{i, t}^{*}$;

(iii) Compute new $\mu^{*}=\mathrm{E}_{g}\left(\mu \mid y ; \xi^{*}\right)$ from the resulting model 12 with $\xi=\xi^{*}, c_{i, t}=c_{i, t}^{*}$ and $d_{i, t}=d_{i, t}^{*}$

(iv) Compute new $\xi^{*}=\mathrm{E}_{g}\left(\xi \mid y ; \mu^{*}\right)$ from the resulting model 12 with $\mu=\mu^{*}, c_{i, t}=c_{i, t}^{*}$ and $d_{i, t}=d_{i, t}^{*}$

(v) Iterate from (ii) to (iv) until convergence.

Since the mode and the mean of the approximating linear Gaussian model are set equal to the mode of the original model, it holds that after convergence $\mu^{*}=\hat{\mu}=\operatorname{argmax}_{\mu} p(\mu \mid y ; \hat{\xi} ; x)$ and $\xi^{*}=\hat{\xi}=\operatorname{argmax}_{\mu} p(\xi \mid y ; \hat{\mu} ; x)$. Further, it holds that $\{\hat{\mu}, \hat{\xi}\}=\operatorname{argmax}_{\mu, \xi} p(\mu, \xi \mid y ; x)$.

The performance of Algorithm A depends crucially on the efficient computation of the conditional expectations in steps (iii) and (v). With respect to step (iii), for a given value of $\xi$, the approximating model 12 is reduced to a standard random effects model as given by

$$
\bar{y}_{i}=\bar{c}_{i}+\bar{A}_{i} \mu_{i}+\overline{\mathcal{B}}_{i}+\bar{u}_{i}, \quad \bar{u}_{i} \sim \operatorname{NID}\left(0, \bar{D}_{i}\right), \quad i=1, \ldots, N
$$

where $\bar{y}_{i}=\left(y_{i, 1}, \ldots, y_{i, T}\right)^{\prime}, \bar{c}_{i}=\left(c_{i, 1}, \ldots, c_{i, T}\right)^{\prime}, \bar{A}_{i}=\left(a_{i, 1}, \ldots, a_{i, T}\right)^{\prime}, \overline{\mathcal{B}}_{i}=\left(b_{i, 1}^{\prime} \xi_{1}, \ldots, b_{i, T}^{\prime} \xi_{T}\right)^{\prime}$ and $\bar{u}_{i}=\left(u_{i, 1}, \ldots, u_{i, T}\right)^{\prime}$, see Baltagi (2005, Chapters 2 and 5$)$. The $T \times T$ variance matrix 
$\bar{D}_{i}$ is diagonal by construction, with elements $d_{i, 1}^{2}, \ldots, d_{i, T}^{2}$ on the main diagonal. Based on (14), the computation of $\mathrm{E}_{g}\left(\mu \mid y ; \xi^{*}\right)$ can be performed using standard multivariate normal regression theory.

In step (v) we need to compute $\mathrm{E}_{g}\left(\xi \mid y ; \mu^{*}\right)$. Given a value of $\mu$, the approximating model (12) can be written as a linear Gaussian state space model as given by

$$
y_{t}=c_{t}+\mathcal{A}_{t}+B_{t} \xi_{t}+u_{t}, \quad u_{t} \sim \operatorname{NID}\left(0, D_{t}\right), \quad t=1, \ldots, T,
$$

where $y_{t}=\left(y_{1, t}, \ldots, y_{N, t}\right)^{\prime}, c_{t}=\left(c_{1, t}, \ldots, c_{N, t}\right)^{\prime}, \mathcal{A}_{t}=\left(a_{1, t}^{\prime} \mu_{1}, \ldots, a_{N, t}^{\prime} \mu_{N}\right)^{\prime}, B_{t}=\left(b_{1, t}, \ldots, b_{N, t}\right)^{\prime}$ and $u_{t}=\left(u_{1, t}, \ldots, u_{N, t}\right)^{\prime}$. Variance matrix $D_{t}$ is diagonal by construction, with elements $d_{1, t}^{2}, \ldots, d_{N, t}^{2}$ on the main diagonal. Based on (15) the computation of $\mathrm{E}_{g}\left(\xi \mid y ; \mu^{*}\right)$ is carried out using the Kalman filter and smoothing methods. Additional computational details are provided in the Technical Appendix.

\subsection{Collapsing the approximate linear Gaussian panel data model}

The evaluation of likelihood estimate $\hat{p}(y)$ in 100 requires $M$ samples of $\mu$ and $\xi$ from importance densities $g(\mu \mid y ; \hat{\xi})$ and $g(\xi \mid y ; \hat{\mu})$, respectively. Both importance densities are based on approximating models given in (14) and (15). However, both models have large dimensions leading to simulation smoother methods that are computationally demanding. Instead, we show that more efficiency can be obtained by first performing two transformations to reduce the cross-section and time series dimensions of observed data $y$. In particular, we show that samples $\xi^{(i)}$ and $\mu^{(i)}$ can be drawn from $g\left(\xi \mid y^{l} ; \hat{\mu}\right)$ and $g\left(\mu \mid \bar{y}^{l} ; \hat{\xi}\right)$, respectively, where $\bar{y}^{l}=\left[\left(\bar{y}_{1}^{l}\right)^{\prime}, \ldots,\left(\bar{y}_{N}^{l}\right)^{\prime}\right]^{\prime}$ and $y^{l}=\left[\left(y_{1}^{l}\right)^{\prime}, \ldots,\left(y_{T}^{l}\right)^{\prime}\right]^{\prime}$ are low-dimensional vector series. The resulting samples can be regarded as coming from $g(\mu \mid y ; \hat{\xi})$ and $g(\xi \mid y ; \hat{\mu})$, respectively.

For the simulation of time-varying effects $\xi^{(i)}$ from $g\left(\xi \mid y^{l} ; \hat{\mu}\right)$, we collapse $N \times 1$ vectors $y_{t}$, based on equation (15), with $\mathcal{A}_{t}$ replaced by $\hat{\mathcal{A}}_{t}=\left(a_{1, t}^{\prime} \hat{\mu}_{1}, \ldots, a_{N, t}^{\prime} \hat{\mu}_{N}\right)^{\prime}$, into low-dimensional $r \times 1$ vectors $y_{t}^{l}$, without losing information relevant for the extraction of $\xi$. A $r \times N$ transformation matrix $S_{t}^{l}$ that satisfies the conditions to prevent information loss is given by 


$$
S_{t}^{l}=\Delta_{t}^{\prime} B_{t}^{\prime} D_{t}^{-1}, \quad \Delta_{t} \Delta_{t}^{\prime}=\left(B_{t}^{\prime} D_{t}^{-1} B_{t}\right)^{-1},
$$

with $\Delta_{t}$ being a lower triangular matrix, see Jungbacker \& Koopman (2008). This choice for $y_{t}^{l}=S_{t}^{l} y_{t}$ gives

$$
y_{t}^{l}=\Delta_{t}^{-1} \xi_{t}+u_{t}^{l}, \quad u_{t}^{l} \sim \mathrm{NID}\left(0, I_{r}\right), \quad t=1, \ldots, T,
$$

where $\Delta_{t}^{-1}$ is a $r \times r$ lower triangular matrix, $\xi_{t}$ is defined in (4) and $u_{t}^{l}$ is a random vector with mean zero and variance equal to the $r$-dimensional unit matrix $I_{r}$. Sampling time-varying effects $\xi^{(i)}$ from $g\left(\xi \mid y^{l} ; \hat{\mu}\right)$ is performed by applying the simulation smoother methods of Durbin \& Koopman (2002) to $r$-dimensional vector series $y_{t}^{l}$ and model (17), for $t=1, \ldots, T$. The high dimensional orthogonal complement of $S_{t}^{l}$ can be constructed from $S_{t}^{l}$ but is not required for any of the necessary computations.

For the simulation of individual-specific effects $\mu^{(i)}$ from $g\left(\mu \mid \bar{y}^{l} ; \hat{\xi}\right)$ we collapse $T \times 1$ vectors $\bar{y}_{i}$, for $i=1, \ldots, N$, based on vector representation 14 , with $\overline{\mathcal{B}}_{i}$ replaced by $\hat{\mathcal{B}}_{i}=\left(b_{i, 1}^{\prime} \hat{\xi}_{1}, \ldots, b_{i, T}^{\prime} \hat{\xi}_{T}\right)^{\prime}$, into $q \times 1$ vectors $\bar{y}_{i}^{l}$. We consider similar least squares type transformations as for the cross-section dimension above. However, because $\mu_{i}$ and $\mu_{j}$ are independent, the transformed observations $\bar{y}_{i}^{l}$ become simple rescaled averages of the variables in $\bar{y}_{i}$. A suitable $T \times q$ transformation matrix $\bar{S}_{i}^{l}$ is given by

$$
\bar{S}_{i}^{l}=\bar{\Delta}_{i}^{\prime} \bar{A}_{i}^{\prime} \bar{D}_{i}^{-1}, \quad \bar{\Delta}_{i} \bar{\Delta}_{i}^{\prime}=\left(\bar{A}_{i}^{\prime} \bar{D}_{i}^{-1} \bar{A}_{i}\right)^{-1}, \quad .
$$

with $\bar{\Delta}_{i}$ being a lower triangular matrix. The resulting model for $\bar{y}_{i}^{l}=\bar{S}_{i}^{l} \bar{y}_{i}$ is given by

$$
\bar{y}_{i}^{l}=\bar{\Delta}_{i}^{-1} \mu_{i}+\bar{u}_{i}^{l}, \quad \bar{u}_{t}^{l} \sim \operatorname{NID}\left(0, I_{q}\right), \quad i=1, \ldots, N,
$$

where $\bar{\Delta}_{i}^{-1}$ is a lower triangular $q \times q$ matrix, $\mu_{i}$ is given in (4) and $\bar{u}_{i}^{l}$ is a random vector with mean zero and $q \times q$ unit variance. Samples $\mu^{(i)}$ can be drawn independently from $g\left(\mu_{i} \mid \bar{y}_{i}^{l} ; \hat{\xi}\right)$, 
which is a Gaussian density with mean $\Sigma_{\mu} \bar{\Delta}_{i}^{-1}\left(\bar{\Delta}_{i}^{-1^{\prime}} \Sigma_{\mu} \bar{\Delta}_{i}^{-1}+I_{q}\right)^{-1} \bar{y}_{i}^{l}$ and variance $\Sigma_{\mu}-$ $\Sigma_{\mu} \bar{\Delta}_{i}^{-1}\left(\bar{\Delta}_{i}^{-1^{\prime}} \Sigma_{\mu} \bar{\Delta}_{i}^{-1}+I_{q}\right)^{-1} \bar{\Delta}_{i}^{-1^{\prime}} \Sigma_{\mu}$. In the Technical Appendix we present the computational details for the transformations and we discuss the computational gains. On average the likelihood evaluation times reduce between 2 and 10 times depending on the different random effects included in the model.

\subsection{Constructing the Monte Carlo likelihood}

Next we discuss the construction of the Monte Carlo likelihood estimate $\hat{p}(y)$ in equation (10). The estimate relies on densities $g(y ; \hat{\mu})$ and $g(y ; \hat{\xi})$, that are based on the approximating model (12). Density $\log g(y ; \hat{\mu})$ can be computed from the prediction error decomposition of vector representation (15), with $\mu$ replaced by $\hat{\mu}$. This is obtained by a single pass through the Kalman filter, see Durbin \& Koopman (2012, Chapter 7). Computational efficiency can increased by using the lower dimensional model (17), based on vector series $y_{t}^{l}$. In particular, Jungbacker \& Koopman (2008) show that

$$
\log g(y ; \hat{\mu})=\text { constant }+\log g\left(y^{l} ; \hat{\mu}\right)-\frac{1}{2} \sum_{t=1}^{T} \log \left|D_{t}\right|+e_{t}^{\prime} D_{t}^{-1} e_{t}
$$

where $y^{l}=\left(y_{1}^{l^{\prime}}, \ldots, y_{T}^{l^{\prime}}\right)^{\prime}$ and $e_{t}=y_{t}-c_{t}-\hat{\mathcal{A}}_{t}-B_{t}\left(B_{t}^{\prime} D_{t}^{-1} B_{t}\right)^{-1} B_{t}^{\prime} D_{t}^{-1}\left(y_{t}-c_{t}-\hat{\mathcal{A}}_{t}\right)$ is the generalized least squares residual vector. Density $g\left(y^{l} ; \hat{\mu}\right)$ can be computed from the prediction error decomposition of model (17), which is a $r \times T$-dimensional problem.

The $\log$ density $\log g(y ; \hat{\xi})$ is also evaluated using the collapsed vector series $\bar{y}_{i}^{l}$, for $i=$ $1, \ldots, N$. Based on model (19) we obtain

$$
\log g(y ; \hat{\xi})=\mathrm{constant}+\log g\left(\bar{y}^{l} ; \hat{\xi}\right)-\frac{1}{2} \sum_{i=1}^{N} \log \left|\bar{D}_{i}\right|+\bar{e}_{i}^{\prime} \bar{D}_{i}^{-1} \bar{e}_{i}
$$

where $\bar{y}^{l}=\left(\bar{y}_{1}^{l^{\prime}}, \ldots, \bar{y}_{N}^{l^{\prime}}\right)^{\prime}$ and $\bar{e}_{i}=\bar{y}_{i}-\bar{c}_{i}-\hat{\mathcal{B}}_{i}-\bar{A}_{i}\left(\bar{A}_{i}^{\prime} \bar{D}_{i}^{-1} \bar{A}_{i}\right)^{-1} \bar{A}_{i}^{\prime} \bar{D}_{i}^{-1}\left(\bar{y}_{i}-\bar{c}_{i}-\hat{\mathcal{B}}_{i}\right)$.

The following algorithm summarizes the evaluation of the loglikelihood for balanced panels. Given parameter vector $\psi$ we can evaluate the Monte Carlo loglikelihood estimate 
$\log \hat{p}(y)$ in the following steps:

\section{Algorithm B}

(i) Run Algorithm A, where the posterior modal values $\hat{\mu}$ and $\hat{\xi}$ are calculated;

(ii) Collapse panel $y$ into low-dimensional vector series $\bar{y}_{i}^{l}$ and $y_{t}^{l}$ using Section 3.2 ,

(iii) Sample $M$ draws $\mu^{(i)}$ and $\xi^{(i)}$ from densities $g\left(\xi \mid y^{l} ; \hat{\mu}\right)$ and $g\left(\mu \mid \bar{y}^{l} ; \hat{\xi}\right)$, which are based on transformed models (17) and (19), and compute importance weights $w^{(i)}$, as given in equation (11);

(iv) Evaluate $\log d e n s i t i e s \log g(y ; \hat{\mu})$ as in 20 and $\log g(y ; \hat{\xi})$ as in 21);

(v) Compute $\log \hat{p}(y)=\log g(y ; \hat{\mu})+\log g(y ; \hat{\xi})+\log M^{-1} \sum_{i=1}^{M} w^{(i)}$.

Loglikelihood estimate $\log \hat{p}(y)$ can be optimized with respect to parameter vector $\psi$ using an arbitrary numerical optimization method. As a practical choice we use the BFGS algorithm, see Nocedal \& Wright (1999). To retain the smoothness of the likelihood in $\psi$ we use the same random seed and the same value of $M$ for each loglikelihood evaluation. The resulting Monte Carlo parameter estimates are denoted by $\tilde{\psi}$. Durbin \& Koopman (1997) advocate the use of antithetic variables to improve the efficiency of the importance sampling weights. An antithetic variable in our context is constructed for each random draw $\mu^{(i)}$ or $\xi^{(i)}$ from the importance densities such that it is equiprobable with $\mu$ or $\xi$, respectively, and it leads to smaller Monte Carlo variation. For each draw of $\mu^{(i)}$ and $\xi^{(i)}$ we manufacture antithetic variables that balance for location and for scale.

\subsection{Unbalanced or incomplete panels}

In this section we provide the details for the treatment of unbalanced panels. We assume that for each individual we observe $y_{i, t}$ and $x_{i, t}$ for $T_{i}$ consecutive time periods during a fixed time interval. When $y_{i, t}$ is unobserved step (ii) of Algorithm A is adjusted by removing $x_{i, t}$ and unobservable lags $\mathcal{Y}_{i, t-1}$ from $z_{i, t}$. The resulting $z_{i, t}$ only depends on $\mu_{i}, \xi_{t}$ and possibly 
observed elements of $\mathcal{Y}_{t-1}$. Calculations in step (iii), conditional on $\xi^{*}$, are based on the standard random effects model, for which missing values can be handled by adopting the methods discussed by Baltagi (2005, Chapter 9). Step (v) of Algorithm A is calculated by Kalman filter and smoothing methods, which can account for missing values, see Durbin \& Koopman (2012, Section 4.10).

The transformations for panel reduction from Section 3.2 need to be adjusted for missing values as well. Jungbacker, Koopman \& van der Wel (2011), show that by choosing an alternative state space representation for model (15) collapsed vectors $y_{t}^{l}$ can be computed using similar transformations. The second transformation for the construction of $\bar{y}_{i}^{l}$ can be computed based on the observed elements of $\bar{y}_{i}$ only, as $\mu_{i}$ and $\mu_{j}$ are independent for all $i, j=1, \ldots, N$.

Likelihood estimate $\hat{p}(y)$ is based on densities $g(y ; \hat{\mu})$ and $g(y ; \hat{\xi})$ and weights $w^{(i)}$. Density $g(y ; \hat{\mu})$ is based on the prediction error decomposition of lower dimensional model (17) and can be computed from the Kalman filter output. Generalized least squares residuals $e_{t}$ only need to be computed for observed elements of $y_{t}$. Density $g(y ; \hat{\xi})$, equation 21 can be computed based on lower dimensional model (19) and by adjusting the generalized least squares residual vectors $\bar{e}_{i}$ to contain only the observed elements of $\bar{y}_{i}$. The weights $w^{(i)}$ in (11) are based on elements of $p\left(y \mid \mu^{(i)}, \xi^{(i)} ; x\right)$ for which $y_{i, t}$ and $x_{i, t}$ are actually observed.

\section{Monte Carlo study}

We present and discuss our results from an extended Monte Carlo study for the generalized dynamic panel data model with the Binary, Binomial and Student's $t$ observation densities. These three models are also considered in our empirical illustrations discussed in Section 5. The purpose of the Monte Carlo study is to evaluate the small sample properties of the estimation procedure presented in Section 3 . In particular, we examine whether a $\sqrt{M}$ convergence rate is likely to exist for our simulated likelihood estimate from Algorithm B. Additionally, we study the magnitude of the Monte Carlo variance of the simulated likelihood 
estimate. Finally, the computational feasibility and accuracy of the estimation methodology is studied.

\subsection{Monte Carlo design}

Our aim is to assess the performance of our simulation-based estimation procedure for a set of different densities, signals, parameter values, panel sizes and numbers of missing values. Table 1 presents the combinations of densities, signals and parameter values, that we investigate in our study. Three different observation densities are considered for (1) and correspond to (A) Binary, (B) Binomial or (C) Student's $t$ distributions. The signals correspond to models with 1 . individual-specific effects or 2. time-varying effects or 3 . both 1. and 2 .

The signal is generated as explained in equations (2) - (4) of Section 2. The fixed component $w_{i, t}$ is based on a single covariate $x_{i, t}$, that is drawn independently from the $\mathrm{N}(0,1)$ distribution, with $\beta=1$ and on the lagged dependent variable with $p_{y}=1$ and $\gamma_{1}=\gamma=0.2$. The stochastic component is based on the univariate, $q=1$, individual-specific effect $\mu_{i}$ and the univariate, $r=1$, time-varying effect $\xi_{t}$ with weights $a_{i, t}=b_{i, t}=1$. The individual-specific effect $\mu_{i}$ is normally distributed with common mean $\delta=0$ and variance $\Sigma_{\mu}=\sigma_{\mu}^{2}$, that is $\mu_{i} \sim \mathrm{N}\left(0, \sigma_{\mu}^{2}\right)$. We investigate the results for different values of standard deviation $\sigma_{\mu}=0.5,1,3$. The time-varying effect is updated by an autoregressive process $\alpha_{t}$ of order 1, where $G=1, H=h, R=1$ and $\Sigma_{\eta}=\sigma_{\eta}^{2}$. Different degrees of persistence are investigated by taking $h=0.3$ or $h=0.9$. The scaling of the time-varying effects is chosen as $\sigma_{\eta}=0.2$.

For the Student's $t$ density we have additional parameters $\nu$ and $\sigma$, where $\nu$ is the degrees of freedom and $\sigma$ is the scaling. We fix the value of $\sigma$ at one and estimate degrees of freedom $\nu$ along with the other parameters. We consider $\nu=3, \nu=5$ and $\nu=10$. The entire parameter vector is given by $\psi=\left\{\gamma, \beta, \sigma_{\mu}, h, \sigma_{\eta}, \nu\right\}$.

We consider five different combinations of the panel dimensions $N, T=10,50,100,250$ In the case of $N=T=10$, the dimensions are sufficiently small that we can also consider 
the standard implementation of Shephard \& Pitt (1997) and Durbin \& Koopman (1997), see Section 4.3. In all other cases, it is computationally only feasible to implement our newly proposed method presented in Section 3.

\subsection{Diagnostic tests for the importance sampler}

A sufficient condition to guarantee a $\sqrt{M}$ convergence rate for the Monte Carlo estimate $\hat{p}(y)$ in 10 is the existence of the variance in the importance samplings weights $w^{(i)}$, as given in equation (11), for $i=1, \ldots, M$, see Geweke (1989). To test whether the variance is finite Koopman et al. (2009) propose test statistics for evaluating variance in a sequence of importance weights. Implementation of their suggested Wald type test statistic is done by the following steps: (1) Simulate a panel y using a combination of observation density, signal, parameter values and panel dimensions as given in Table 1. (2) Estimate the parameters using the Monte Carlo maximum likelihood methods of Section 3 and replace the parameter vector $\psi$ by the resulting estimate $\tilde{\psi}$. (3) Generate 100, 000 importance sampling weights $w^{(i)}$ using the importance densities $g\left(\xi \mid y^{l} ; \hat{\mu}\right)$ and $g\left(\mu \mid \bar{y}^{l} ; \hat{\xi}\right)$. (4) Consider $s$ exceedence sampling weights, denoted by $x_{1}, \ldots, x_{s}$, which are larger than some threshold $w^{\text {min }}$ and are assumed to come from the generalized Pareto distribution with logdensity function $f(a, b)=-\log b-$ $\left(1+a^{-1}\right) \log \left(1+a b^{-1} x_{i}\right)$ for $i=1, \ldots, s$, where unknown parameters $a$ and $b$ determine the shape and scale of the density, respectively. When $a \leq 0.5$, the variance of the importance sampling weights exists and a $\sqrt{M}$ convergence rate can be assumed. (5) Estimate $a$ and $b$ by maximum likelihood and denote the estimates by $\tilde{a}$ and $\tilde{b}$, respectively. (6) Compute the t-test statistic $t_{w}=\tilde{b}^{-1} \sqrt{s / 3}(\tilde{a}-0.5)$ to test the null hypothesis $H_{0}: a=0.5$. We reject the null hypothesis when the statistic is positive and significantly different from zero, that is, when it is larger than 1.96 with $95 \%$ confidence.

Figures 1(a) and 1(b) present the diagnostic test statistics for the importance samplers applied to three different densities (A, B and C), and four signals (1.b, 2.b, 3.b and 3.e) as listed in Table 1. Furthermore, the test statistics are computed for different values of the threshold $w^{\text {min }}$. We choose $w^{\text {min }}$ values such that a range of the largest $1 \%$ to $50 \%$ of the 
Observation density

\begin{tabular}{l} 
(A) Binary $\quad \log p\left(y_{i, t} \mid z_{i, t}\right)=y_{i, t} z_{i, t}-\log \left(1+\exp z_{i, t}\right)$ \\
(B) Binomial $\log p\left(y_{i, t} \mid z_{i, t}\right)=y_{i, t} z_{i, t}-n_{i, t}\left(1+\exp z_{i, t}\right)-\log \left(\begin{array}{l}n_{i, t} \\
y_{i, t}\end{array}\right)$ \\
(C) Student's $t \log p\left(y_{i, t} \mid z_{i, t}\right)=\log a(\nu)+\frac{1}{2} \log \lambda-\frac{\nu+1}{2} \log \left(1+\lambda\left(y_{i, t}-z_{i, t}\right)^{2}\right)$ \\
\hline
\end{tabular}

\begin{tabular}{llllllll} 
Signal & Parameters \\
\hline & $\gamma$ & $\beta$ & $\sigma_{\mu}$ & $h$ & $\sigma_{\eta}$ & $\nu$ \\
\hline
\end{tabular}

(1) $\quad z_{i, t}=y_{i, t-1} \gamma+x_{i, t}^{\prime} \beta+\mu_{i}$

$\begin{array}{lllllll}\text { a) } & 0.2 & 1 & 0.5 & - & - & (3,5,10) \\ \text { b) } & 0.2 & 1 & 1 & - & - & (3,5,10) \\ \text { c) } & 0.2 & 1 & 3 & - & - & (3,5,10)\end{array}$

$(2)$

$$
z_{i, t}=y_{i, t-1} \gamma+x_{i, t}^{\prime} \beta+\xi_{t} \quad \text { a) } \quad 0.2 \quad 1 \quad-\quad 0.3 \quad 0.2 \quad(3,5,10)
$$

b) $\quad 0.2 \quad 1 \quad-\quad 0.9 \quad 0.2 \quad(3,5,10)$

$(3)$

$$
\begin{array}{rlllllll}
z_{i, t}=y_{i, t-1} \gamma+x_{i, t}^{\prime} \beta+\mu_{i}+\xi_{t} & \text { a) } & 0.2 & 1 & 0.5 & 0.3 & 0.2 & (3,5,10) \\
& \text { b) } & 0.2 & 1 & 0.5 & 0.9 & 0.2 & (3,5,10) \\
& \text { c) } & 0.2 & 1 & 1 & 0.3 & 0.2 & (3,5,10) \\
& \text { d) } & 0.2 & 1 & 1 & 0.9 & 0.2 & (3,5,10) \\
& \text { e) } & 0.2 & 1 & 3 & 0.3 & 0.2 & (3,5,10) \\
\text { f) } & 0.2 & 1 & 3 & 0.9 & 0.2 & (3,5,10)
\end{array}
$$

Table 1: Monte Carlo design with our signal specifications, parameter values and panel dimensions for simulating the observations. The data generation process is further given by $x_{i, t} \sim \operatorname{NID}(0,1)$, $\mu_{i} \sim \mathrm{NID}\left(0, \sigma_{\mu}^{2}\right), \xi_{t}=\alpha_{t}, \alpha_{t+1}=h \alpha_{t}+\eta_{t}$ and $\eta_{t} \sim \mathrm{NID}\left(0, \sigma_{\eta}^{2}\right)$. The initial time varying effect is taken as $N\left(0, \sigma_{\eta}^{2} /\left(1-h^{2}\right)\right)$. For the Student's $t$ density it holds that $a(\nu)=\Gamma(\nu / 2+1 / 2) / \Gamma(\nu / 2)$ and $\lambda^{-1}=(\nu-2) \sigma^{2}$. 

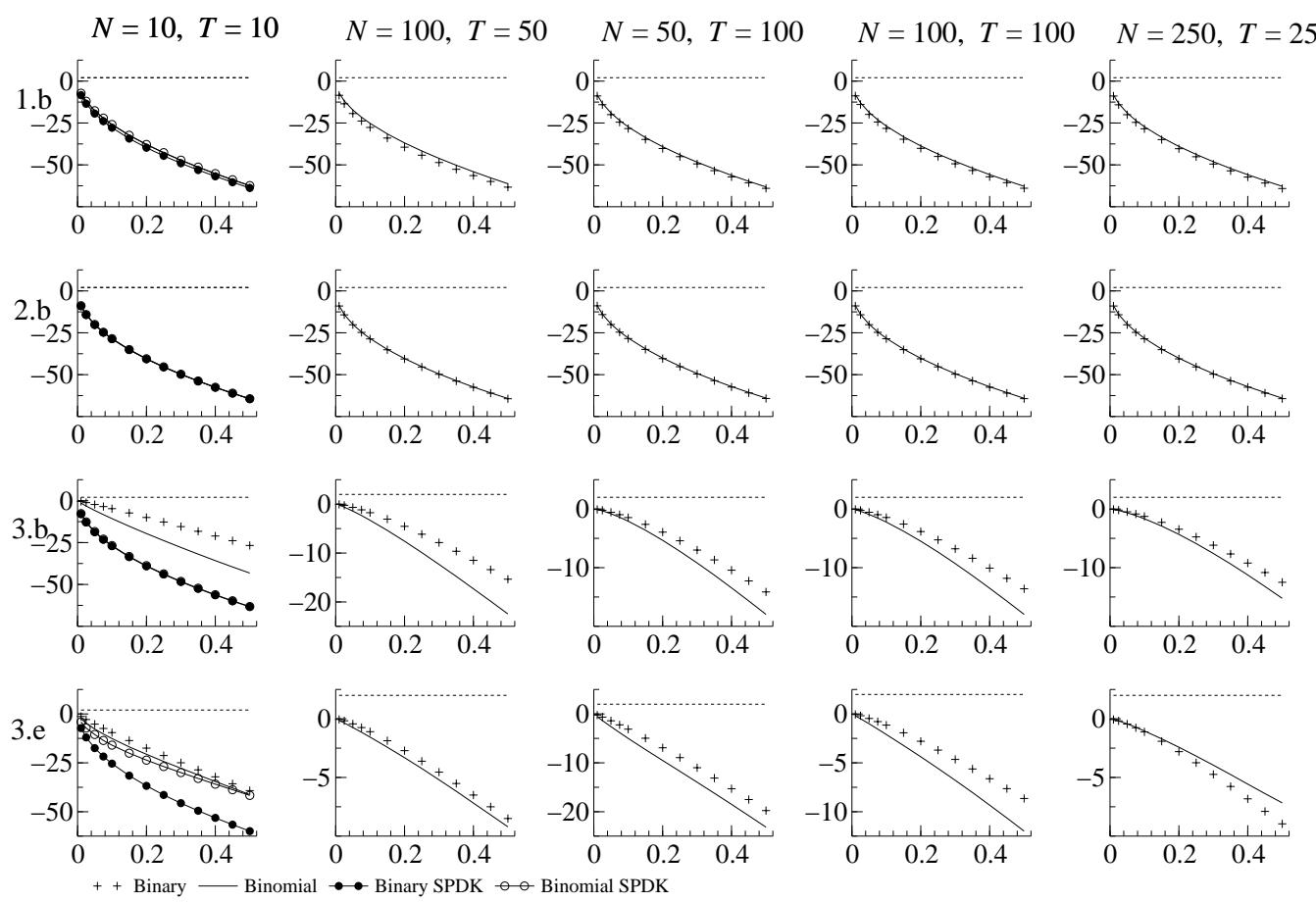

(a) Binary and Binomial
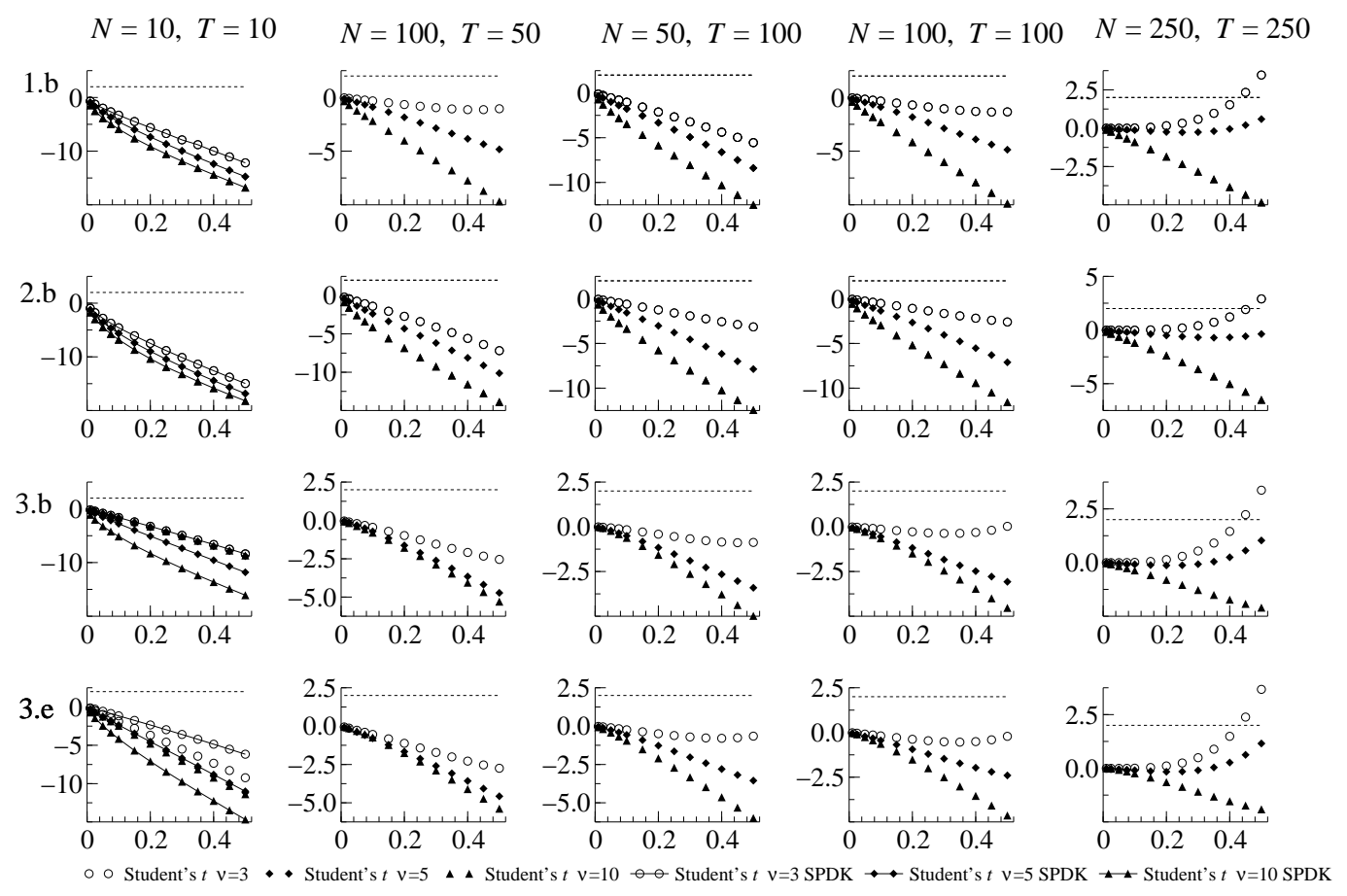

(b) Student's $t$

Figure 1: Importance sampling diagnostics for dynamic panel data models, based on 100,000 simulations of weights $w^{(i)}$ defined in equation (11). The test statistics are presented for densities A Binary and B Binomial, and signals 1.b, 2.b, 3.b and 3.e from Table 1 and for different panel sizes. For each combination we computed test statistics for different thresholds $w^{\mathrm{min}}$, by procedures explained in Section 4.2. Thresholds are based on the number of exceedence values $x_{1}, \ldots, x_{s}$ included. We have taken $0.01=s / 100000,0.025=s / 100000,0.05=s / 100000$, until $0.5=$ $s / 100000$. The area above the dotted straight line at 2 indicates the rejection area. 
weights are included. The test statistics are computed with the use of antithetic variables in all cases.

The test statistics in Figure 1(a) for the Binary and Binomial densities are negative for all combinations of signals, parameter values and panel dimensions. They provide strong evidence for the existence of a variance for the importance density function. The test statistics in Figure 1(b) for the Student's $t$ density present a similar picture. However, when the degrees of freedom is small, $\nu=3$, and the panel dimensions are large $N=250$ and $T=250$, the test is rejected for threshold values that lead to exceedence samples that include more than $35 \%$ of all weights. When the degrees of freedom becomes even smaller similar results are obtained. We notice that only for large exceedance samples, we move away from the left-tail of the test statistic distribution.

We may conclude that there is substantial evidence that a $\sqrt{M}$ convergence rate exists for our proposed likelihood estimate for the generalized dynamic panel data model. However we do recommend to check for each application whether the importance weights are finite. The presented results also hold for the other signals listed in Table 1. They are further discussed in the Technical Appendix.

\subsection{Measuring the efficiency loss of Algorithm A}

We discussed in Section 3 that our proposed implementation of importance sampling for the generalized dynamic panel data model is different from the standard implementation of Shephard \& Pitt (1997) and Durbin \& Koopman (1997), hereafter SPDK. The standard SPDK method would sample $\mu^{(i)}$ and $\xi^{(i)}$ from $g(\mu, \xi \mid y)$, where $g(\mu, \xi \mid y)$ is based on the linear Gaussian model $(12)$. The corresponding likelihood is then estimated by

$$
\hat{p}(y)=g(y) M^{-1} \sum_{i=1}^{M} w^{(i)}, \quad w^{(i)}=\frac{p\left(y \mid \mu^{(i)}, \xi^{(i)} ; x\right)}{g\left(y \mid \mu^{(i)}, \xi^{(i)}\right)}
$$

where $\mu^{(i)}$ and $\xi^{(i)}$ are drawn from $g(\mu, \xi \mid y)$. This implementation is not feasible for even moderately large panels, see the discussion in Section 3 . 
It is anticipated that the variance of the importance weight function increases when the SPDK method in (22) is replaced by our method in (10) because Algorithm A does not account for the dependence between $\mu$ and $\xi$ in $g(\mu, \xi \mid y)$. To study the extent of the increase in the variance, we compare the t-test statistics for the importance weights in (11) with importance weights in 22 . We notice that sampling from the SPDK importance density is only computationally feasible for small panel sizes and that the collapsing method of Section 3.2 is not applicable to $g(\mu, \xi \mid y)$.

For the small panel $N=10$ with $T=10$, we have implemented the standard SPDK method for our models to facilitate its comparison with our proposed method. Hence Figures 1(a) and 1(b) also present the diagnostic test statistics of Section 4.2 for the SPDK method and for the smallest panel only. The differences between the test statistics for SPDK and Algorithm A are small. For all three models, the test statistics for the SPDK importance weights are slightly lower, but both are very negative. We notice that SPDK is clearly not feasible for even modest panel dimensions.

In the Technical Appendix we also provide estimates for the loss of efficiency in the likelihood that results from the increase in variance. We document that the loss can be substantial for the Binary and Binomial densities, but low for the Student's $t$ density. However, the increase of variance in the likelihood estimate can be offset by increasing the number of draws $M$. Any degree of accuracy can be achieved and given the large computational improvements that are also documented in the Technical Appendix, this is a minor difficulty. In the remainder of this paper we always take $M=1000$ draws from the importance density of Algorithm A.

\subsection{Finite sample properties of Algorithm B}

We investigate the accuracy of the likelihood estimates by simulating 100 data panels for each possible design given in Table 1. For each simulated data panel, we estimate the parameter vector $\psi$ using Algorithm B with collapsed vectors. After collecting all estimated parameter vectors, we report the average bias and standard deviation in Table 2 for signals 


\begin{tabular}{|c|c|c|c|c|c|c|c|c|}
\hline & $N$ & $T$ & $\gamma$ & $\beta$ & $\sigma_{\mu}$ & $h$ & $\sigma_{\eta}$ & $\nu$ \\
\hline \multirow[t]{4}{*}{ A.3.b } & 100 & 50 & $-0.004_{0.072}$ & $0.001_{0.036}$ & -0.0020 .057 & $-0.057 \quad 0.127$ & $0.001_{0.042}$ & - \\
\hline & 50 & 100 & -0.0060 .065 & -0.0040 .041 & -0.0120 .058 & -0.0320 .085 & -0.0010 .038 & - \\
\hline & 100 & 100 & 0.0040 .045 & 0.0040 .032 & -0.0040 .043 & -0.0340 .081 & 0.0020 .037 & - \\
\hline & 250 & 250 & -0.0010 .019 & -0.0000 .010 & -0.0040 .024 & -0.0070 .035 & -0.0010 .016 & - \\
\hline \multirow[t]{4}{*}{ A.3.e } & 100 & 50 & 0.0040 .100 & 0.0040 .046 & $\begin{array}{lll}-0.028 & 0.271\end{array}$ & -0.0270 .379 & -0.0330 .096 & - \\
\hline & 50 & 100 & -0.0190 .100 & -0.0010 .052 & -0.0550 .303 & -0.0590 .357 & -0.0150 .073 & - \\
\hline & 100 & 100 & -0.0030 .061 & 0.0030 .035 & -0.0210 .234 & $-0.040 \quad 0.325$ & -0.0190 .067 & - \\
\hline & 250 & 250 & 0.0020 .029 & 0.0010 .013 & -0.0200 .138 & -0.0190 .112 & -0.0000 .018 & - \\
\hline \multirow[t]{4}{*}{ B.3.b } & 100 & 50 & 0.0030 .012 & -0.0010 .023 & -0.0110 .037 & -0.0420 .092 & -0.0000 .030 & - \\
\hline & 50 & 100 & 0.0020 .013 & 0.0020 .021 & -0.0090 .047 & -0.0190 .056 & 0.0020 .027 & - \\
\hline & 100 & 100 & 0.0000 .009 & 0.0020 .014 & $0.000_{0.037}$ & -0.0260 .064 & -0.0010 .021 & - \\
\hline & 250 & 250 & 0.0000 .003 & 0.0010 .005 & -0.0010 .024 & -0.0060 .031 & -0.0010 .010 & - \\
\hline \multirow[t]{4}{*}{ B.3.e } & 100 & 50 & 0.0020 .018 & 0.0050 .028 & -0.0130 .224 & $-0.034 \quad 0.207$ & -0.0050 .037 & - \\
\hline & 50 & 100 & 0.0050 .020 & $\begin{array}{ll}-0.001 & 0.027\end{array}$ & -0.0210 .300 & -0.0450 .200 & -0.0050 .037 & - \\
\hline & 100 & 100 & 0.0020 .013 & 0.0000 .019 & 0.0020 .225 & -0.0350 .160 & -0.0050 .025 & - \\
\hline & 250 & 250 & $0.000 \quad 0.004$ & $0.000 \quad 0.007$ & -0.0080 .142 & $0.000 \quad 0.073$ & -0.0010 .010 & - \\
\hline \multirow[t]{4}{*}{ C.3.b } & 100 & 50 & -0.0010 .008 & -0.0020 .009 & -0.0110 .030 & -0.0180 .040 & 0.0020 .022 & $0.010_{0.051}$ \\
\hline & 50 & 100 & -0.0010 .009 & -0.0030 .009 & -0.0120 .040 & -0.0170 .054 & -0.0030 .023 & 0.0080 .050 \\
\hline & 100 & 100 & 0.0000 .005 & $-0.000 \quad 0.007$ & $\begin{array}{ll}-0.000 & 0.025\end{array}$ & -0.0120 .035 & 0.0020 .015 & $\begin{array}{ll}-0.002 & 0.038\end{array}$ \\
\hline & 250 & 250 & 0.0000 .002 & 0.0000 .003 & 0.0000 .011 & $-0.000 \quad 0.025$ & 0.0000 .010 & 0.0020 .015 \\
\hline \multirow[t]{4}{*}{ C.3.e } & 100 & 50 & -0.0010 .008 & -0.0020 .009 & -0.0370 .168 & -0.0290 .124 & -0.0000 .020 & $0.010_{0.051}$ \\
\hline & 50 & 100 & -0.0010 .008 & -0.0030 .009 & -0.0490 .226 & -0.0010 .107 & -0.0040 .020 & 0.0100 .050 \\
\hline & 100 & 100 & 0.0000 .005 & $-0.000 \quad 0.007$ & 0.0150 .261 & -0.0120 .097 & $0.000 \quad 0.021$ & -0.0020 .039 \\
\hline & 250 & 250 & $0.000 \quad 0.002$ & 0.0010 .003 & -0.0090 .057 & -0.0060 .041 & $0.000 \quad 0.007$ & $\begin{array}{ll}0.001 \quad 0.012 \\
\end{array}$ \\
\hline \multirow[t]{4}{*}{ C.3.b } & 100 & 50 & -0.0010 .009 & 0.0050 .013 & -0.0140 .090 & -0.0670 .090 & -0.0010 .027 & $0.053_{0.244}$ \\
\hline & 50 & 100 & $\begin{array}{ll}-0.001 & 0.008\end{array}$ & 0.0050 .014 & -0.0090 .111 & -0.0380 .072 & $\begin{array}{ll}-0.001 & 0.018\end{array}$ & $\begin{array}{ll}0.050 & 0.222\end{array}$ \\
\hline & 100 & 100 & 0.0010 .005 & -0.0020 .010 & -0.0340 .083 & -0.0170 .055 & -0.0030 .018 & 0.0430 .154 \\
\hline & 250 & 250 & $0.000 \quad 0.002$ & 0.0010 .002 & -0.0170 .037 & -0.0200 .030 & 0.0030 .009 & 0.0290 .040 \\
\hline \multirow[t]{4}{*}{ C.3.e } & 100 & 50 & -0.0020 .008 & 0.0040 .013 & -0.0320 .266 & $\begin{array}{ll}-0.081 & 0.177\end{array}$ & -0.0030 .022 & 0.0520 .239 \\
\hline & 50 & 100 & $-0.000 \quad 0.007$ & 0.0050 .014 & 0.0180 .341 & -0.0240 .130 & -0.0050 .016 & $0.055 \quad 0.222$ \\
\hline & 100 & 100 & $0.000 \quad 0.005$ & $\begin{array}{ll}-0.002 & 0.010\end{array}$ & $\begin{array}{ll}-0.089 & 0.247\end{array}$ & -0.0080 .110 & -0.0040 .018 & 0.0460 .155 \\
\hline & 250 & 250 & 0.0000 .003 & 0.0010 .002 & -0.0370 .117 & -0.0190 .053 & 0.0020 .009 & $0.028 \quad 0.042$ \\
\hline
\end{tabular}

Table 2: Simulation results for the non-Gaussian dynamic panel data models. We present the average bias and in lower case the standard deviation of the parameter estimates resulting from 100 repetitive estimates from different simulated data panels. Specifications 3.b and 3.e from Table 1 together with observation models A, B and C are used for simulation. The third panel corresponds to the Student's $t$ density with $\nu=3$ degrees of freedom whereas the fourth panel corresponds to the Student's $t$ density with $\nu=5$ degrees of freedom All parameters are estimated by procedures outlined in Section 3, with $M=1000$ draws from importance densities $g\left(\xi \mid y^{l} ; \hat{\mu}\right)$ and $g\left(\mu \mid \bar{y}^{l} ; \hat{\xi}\right)$, respectively. 
3.b and 3.e. The results of the Monte Carlo study show that the estimation procedure is successful. All parameter estimates center around their "true" values for all different models and parameter values. We notice that individual state dependence as captured by $\gamma(B) y_{i, t}$ can be empirically identified and separated from stochastic components $\mu_{i}$ and $\xi_{t}$.

For each simulated data panel we also create unbalanced panels by removing $40 \%$ of the observations, at the beginning and end of the data set. The parameter estimates remained unbiased and the standard errors increase slightly due to the loss of data. The full set of parameter estimation results, with and without missing values, are presented in our Technical Appendix. For all computations in this study, we have written the code in the $0 \mathrm{x}$ programming language version 6.10 of Doornik (2007).

\section{$5 \quad$ Empirical illustrations}

\subsection{Union Participation of young males}

We first analyse the union membership decision of young males, see Vella \& Verbeek (1998), Wooldridge (2005) and Liesenfeld \& Richard (2008). We use the data from Vella \& Verbeek (1998) to estimate a logistic model for union membership. The binary outcome of the union membership decision for individual $i$ in year $t$ is denoted by $y_{i, t} \in\{0,1\}$. The data consists of $N=545$ males and their possible membership is yearly observed between 1981 and 1987, $T=7$. The initial time period $t=0$ corresponds to 1980 and the panel is balanced.

The model for the union membership decision is given by density (A) and signal 3 in Table 1. For this signal the coefficient $\gamma$ captures the effect whether the individual is a current union member or not. The explanatory variable vector $x_{i, t}$ includes variables capturing, schooling, experience, marriage, ethnicity, marital status and location effects, see Table 1 in Vella \& Verbeek (1998) for more detailed descriptions of these variables. We depart from the study of Vella \& Verbeek (1998) by not including the industry and occupational dummy variables in $x_{i, t}$ and by replacing their time dummies with our stochastically time-varying effect $\xi_{t}$. 
We conduct three analysis. First, we aim to assess whether the persistence is a result from either individual satisfaction of last year's decision as captured by $\gamma y_{i, t-1}$, referred to as true state dependence, or from underlying aspects that cause the observed persistence due to individual-specific $\mu_{i}$ and time-varying $\xi_{t}$ effects, referred to as spurious state dependence. Second, we recognize that the initial observations and the time-varying explanatory variables tend to be correlated with the individual-specific effect $\mu_{i}$. For example, the marriage decision partly explains the union choice, but may also influence other unobserved characteristics that determine $\mu_{i}$. To address this issue we allow the individual effect to be correlated with the initial observations $y_{i, 0}$ and the time-varying explanatory variables $x_{i, t}$. In particular, we implement the method of Chamberlain (1980) which has been extended for nonlinear dynamic panel data models by Wooldridge (2005) and specify $\mu_{i}$ as

$$
\mu_{i}=y_{i, 0} \lambda_{0}+\sum_{t=1}^{T} x_{i, t}^{\prime} \lambda_{t}+v_{i} \quad v_{i} \sim \operatorname{NID}\left(\delta, \sigma_{\mu}^{2}\right),
$$

where $\lambda_{0}, \lambda_{1}, \ldots, \lambda_{T}$, with $T=7$, are fixed parameters that measure the correlation between $\mu_{i}$ and the predetermined variables, and where $v_{i}$ represents the remainder individual-specific effect with fixed mean $\delta$ and variance $\sigma_{\mu}^{2}$. Third and finally, we consider a different strategy for capturing spurious state dependence that is discussed in Heiss (2008). Here individualspecific time-varying stochastic trends are considered to capture the serial correlation in $\epsilon_{i, t}$. It makes the use of time-invariant individual-specific effects $\mu_{i}$ redundant. In our setting we can implement it as

$$
\epsilon_{i, t}=b_{i}^{\prime} \xi_{t}+y_{i, 0} \lambda_{0}+\sum_{t=1}^{T} x_{i, t}^{\prime} \lambda_{t}
$$

where $\xi_{t}$ is the $(N+1) \times 1$ vector of time varying effects and $b_{i}$ is the $(N+1) \times 1$ selection vector with the $i$ th and the $(N+1)$ th elements set equal to one and zero otherwise, with $N=545$. The first $N$ time-varying effects are specific for each time series while the $(N+1)$ th element is common for all time series. The estimation method suggested in Heiss (2008) does not allow for the common time-varying effect. Following Heiss (2008), we model the first $N$ elements by $\xi_{i, t+1}=g \xi_{i, t}+\eta_{i, t}$, with $|g| \leq 1 \eta_{i, t} \sim \operatorname{NID}\left(\delta(1-g), \sigma_{\lambda}^{2}\left(1-g^{2}\right)\right)$. The $(N+1)$ th 
element is modeled by $\xi_{N+1, t+1}=h \xi_{N+1, t}+\eta_{N+1, t}$, with $|h|<1$ and $\eta_{N+1, t} \sim \operatorname{NID}\left(0, \sigma_{\eta}^{2}\right)$, which is similar as in the original model. When $g=1$ it holds that $\xi_{i, 1} \sim N\left(\delta, \sigma_{\lambda}^{2}\right)$ and $\xi_{i, t}=\xi_{i, t-1}$ for $t>1$, and the model collapses to the original model but with $\xi_{i, 1}$ replacing $v_{i}$ in (23). We can now formally establish whether time-invariant individual-specific effects are appropriate.

The results for the three model specification are presented in Table 3 . The parameter estimates are similar compared to the estimates reported in Vella \& Verbeek (1998) and Liesenfeld \& Richard (2008). The state dependence parameter $\gamma$ is strongly significant. The estimated effects of marriage and being Black/Hispanic are positive and significant while those for other explanatory variables are not significant. The distribution of the individualspecific effects indicates that large differences exist between individuals.

When we allow for correlated random effects via (23) we find that the variable for marriage in 1987 is significantly correlated with the individual-specific effect. The state dependence parameter is estimated smaller for the extended model. However a large part of the dependence is attributed to the correlation between the individual-specific effect and the initial conditions $y_{i, 0}$ as measured by $\lambda_{0}$. Similar changes for the state dependence and marriage parameters are documented in Wooldridge (2005).

For the third model that allows for time-varying individual-specific effects (24) we find that the parameter $g$ is estimated very close to 1 while the estimate of $\sigma_{N, \eta}$ is relatively small. This may partly be due to the relatively small time series dimension $T=7$. For this application we may conclude that including a time-invariant individual-specific effect $\mu_{i}$ is sufficient. For a larger $T$ we may find more evidence of time-varying effects. The other parameter values have similar estimates as for Models 1 and 2.

\subsection{Crime rates between 1930 and 2005}

The general credit (or default) risk modeling frameworks of Duffie, Saita \& Wang (2007) and Koopman \& Lucas (2008) can be applied to other areas of empirical research where the prediction of unattractive events is of key interest. In this empirical illustration we 


\begin{tabular}{|c|c|c|c|}
\hline Variable & Model 1 & Model 2 & Model 3 \\
\hline$y_{i, t-1}$ & $2.344_{0.167}$ & $1.770_{0.153}$ & 1.7820 .157 \\
\hline log Experience & -0.2010 .236 & -0.2500 .200 & -0.2520 .480 \\
\hline Schooling & -0.0200 .054 & -0.0300 .058 & -0.0280 .065 \\
\hline Married & 0.3820 .148 & $0.291_{0.196}$ & 0.2940 .198 \\
\hline Black & $1.124_{0.268}$ & $0.990 \quad 0.286$ & 1.0080 .290 \\
\hline Hispanic & $0.518_{0.257}$ & $0.326 \quad 0.278$ & $0.336 \quad 0.281$ \\
\hline Rural & 0.0230 .196 & 0.0210 .211 & 0.0190 .212 \\
\hline Health & -0.8650 .528 & -0.7300 .541 & $-0.724 \quad 0.547$ \\
\hline North-East & $0.364 \quad 0.273$ & 0.2590 .295 & 0.2630 .300 \\
\hline South & 0.0230 .248 & -0.0180 .269 & $-0.004 \quad 0.282$ \\
\hline North Central & $0.490 \quad 0.263$ & $0.411_{0.285}$ & $0.424 \quad 0.213$ \\
\hline$y_{i, 0}$ & - & 2.1370 .242 & $2.166 \quad 0.231$ \\
\hline Married $_{i, 1}$ & - & $\begin{array}{lll}0.115 & 0.326\end{array}$ & 0.1130 .331 \\
\hline Married $_{i, 2}$ & - & -0.1450 .387 & $\begin{array}{ll}-0.135 & 0.317\end{array}$ \\
\hline Married $_{i, 3}$ & - & -0.1040 .390 & -0.1020 .399 \\
\hline Married $_{i, 4}$ & - & 0.0450 .422 & 0.0330 .415 \\
\hline $\operatorname{Married}_{i, 5}$ & - & 0.5790 .397 & 0.5910 .422 \\
\hline Married $_{i, 6}$ & - & $0.248 \quad 0.397$ & 0.2470 .400 \\
\hline Married $_{i, 7}$ & - & -0.6530 .311 & -0.6530 .316 \\
\hline$h$ & -0.4180 .566 & $-0.271_{0.606}$ & -0.5140 .610 \\
\hline$\sigma_{\eta}$ & $0.126 \quad 0.069$ & 0.0030 .059 & 0.1170 .064 \\
\hline$g$ & - & - & 0.9890 .012 \\
\hline$\sigma_{N, \eta}$ & - & - & $0.040 \quad 0.003$ \\
\hline$\delta$ & -2.4400 .923 & -2.6140 .910 & -2.6811 .603 \\
\hline$\sigma_{\mu}$ & 1.3730 .131 & 1.4860 .113 & $1.514_{0.182}$ \\
\hline $\log \hat{p}(y)$ & -4863.1 & -4808.8 & -4798.9 \\
\hline AIC & 9756.3 & 9663.6 & 9645.9 \\
\hline
\end{tabular}

Table 3: Estimation results (and standard errors in lower case) for the logistic models for the union membership decision of young males. The binary data panel has cross-section dimension $N=545$ and time series dimension $T=7$. Model 1 is our basic model with $y_{i, 0}$ treated as fixed. Model 2 extends Model 1 with (23) for the individual-specific effect $\mu_{i}$. Model 3 implements the stochastic component as in (24). 
consider modeling criminal behavior, see Koopman, Ooms, Lucas, Montfort \& Van der Geest (2008) for an earlier example. We analyze crime panel data from the so-called TRANS-5 study, see Bijleveld \& Wijkman (2009) for a detailed description. The data panel consists of observations for $N=188$ high-risk families from The Netherlands that reaches over five generations between the years 1930 and 2005, $T=75$. For all individuals from these families, 6039 in total, their committed crimes are registered as well as age and gender information. The size of the families fluctuates over time.

In this illustration, we aggregate the data at the family level and let $y_{i, t}$ denote the number of crimes committed by family $i$ in year $t$. The observation $y_{i, t}$ is modeled by the Binomial density as given by density (B) in Table 1, with $n_{i, t}$ being the number of family members, and signal 3. The family-specific effect $\mu_{i}=y_{i, 0} \lambda_{0}+v_{i}$, with $v_{i} \sim N I D\left(\delta, \sigma_{\mu}^{2}\right)$, and the common time-varying effect $\xi_{t}$ follows a random walk $(h=1)$. The common time-varying effect possibly accounts for the general climate in the justice and police systems. The signal $z_{i, t}$ includes explanatory variables that represent the age and gender composition of the family. The current model extends the non-Gaussian state space framework of Koopman \& Lucas (2008) and Koopman et al. (2008) by including the individual-specific means $\mu_{i}$. This is an important extension as typically large differences in propensity exist between families.

The parameter estimates are presented in Table 4. The state dependence coefficient $\gamma$ is significantly estimated and has a positive value, indicating strong positive correlation between past and future criminal behavior within families. The proportion of males in the family has a strongly significant and positive effect on the crime intensity in the family. The estimated coefficients for the age variables, which are constructed as the fraction of individual family members within each age group, reach their peak in the late teenage years after which they slowly decline.

We extend our study by investigating whether the age effects are changing over time. Since crime reducing factors such as marriage and employment have taken place much earlier in life in the earlier years of our sample, we may expect shifts in the age effects. We can investigate this feature by considering time-varying effects for the age regression coefficients. 


\begin{tabular}{|c|c|c|}
\hline Variable & Model 1 & Model 2 \\
\hline$y_{i, t}$ & $0.195 \quad 0.021$ & $0.193 \quad 0.027$ \\
\hline$\%$ males & $1.468 \quad 0.244$ & 0.8530 .051 \\
\hline Age $12-17$ & $1.770_{0.233}$ & $1.370 \quad 0.037$ \\
\hline Age 18-24 & $2.214_{0.189}$ & 2.1940 .041 \\
\hline Age 25-34 & $\begin{array}{ll}1.351 & 0.213\end{array}$ & 0.6190 .031 \\
\hline Age 35-44 & $0.564_{0.253}$ & $0.451 \quad 0.034$ \\
\hline$y_{i, 0}$ & $0.068 \quad 0.044$ & $0.052 \quad 0.029$ \\
\hline$\sigma_{\eta}$ & 0.0580 .020 & $0.019_{0.028}$ \\
\hline$\delta$ & -6.8850 .093 & $-6.734_{0.041}$ \\
\hline$\sigma_{v}$ & $0.960 \quad 0.084$ & 0.9150 .026 \\
\hline $\log \hat{p}(y)$ & -28033 & -24856 \\
\hline AIC & 56084 & 49738 \\
\hline
\end{tabular}

Table 4: Estimation results (and standard errors in lower case) for the crime application. The panel consists of the number of crimes committed by $N=188$ families between 1930 and 2005, $T=75$. Model 1 is the basic model specification. Model 2 extends Model 1 by also considering time-varying effects for the age coefficients.

We replace the common time-varying effect by

$$
b_{i, t}^{\prime} \xi_{t} \text {, where } b_{i, t}=\left(1, \text { Age12-17 }{ }_{i, t}, \text { Age18-24 }{ }_{i, t}, \text { Age25-34 }_{i, t}, \text { Age35-44 } 4_{i, t}\right),
$$

where $\xi_{t}$ is here a $5 \times 1$ vector that captures the common time-varying factor and the timevarying coefficients for age. Each element of $\xi_{t}$ is modeled by a random walk with different standard deviations. The last four initial elements in $\xi_{1}$ are fixed at zero since we also include the age variables in $x_{i, t}$. It allows us to separate the mean age effects from the time-varying effects, see Hsiao \& Pesaran (2008). The estimates for the age effects are shown in Figure 2. We find that the variable for the age group 25-34 increases significantly from say 1980 onwards. This corresponds to an age crime curve that is decaying more slowly after its peak. Hence we may conclude that during the more recent years, the crime reducing factors (marriage and employment) are considered later in life when compared to say the 1930-1950 period. Some evidence of this effect may also be observed for the age group 35-44 but the increase is more steadily and strictly not significant. The developed methodology in this paper has provided the means to extract such interesting features from the data. 

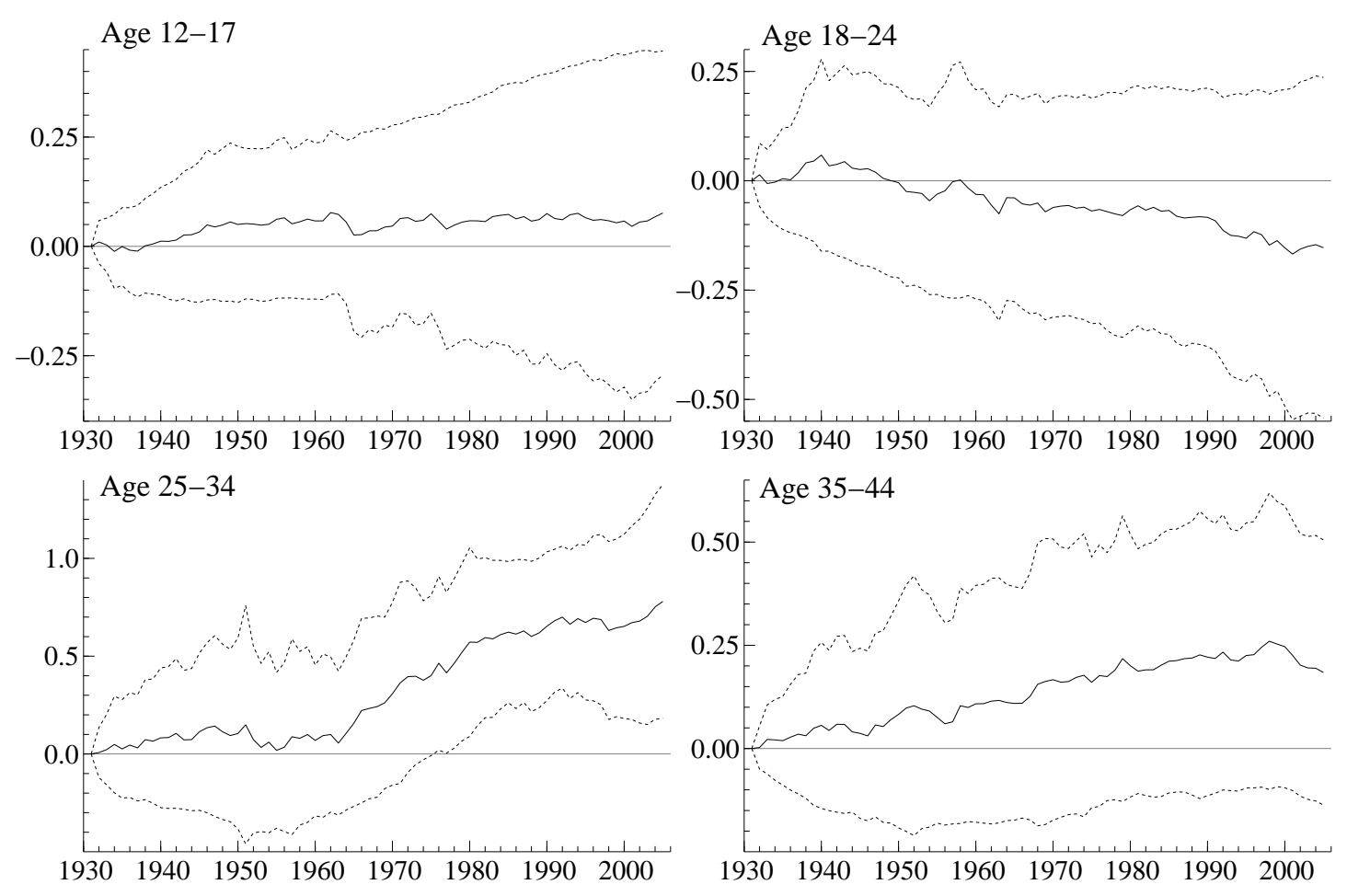

Figure 2: Time-varying effects of the age coefficients for the age crime curve.

\subsection{Economic Growth}

Many studies in the econometric growth literature have the aim to identify determinants of long run economic growth and to develop various measures and tests of growth convergence, see Durlauf, Johnson \& Temple (2005) for an extensive review of the literature. We revisit the stochastic Solow growth model that is derived in Binder \& Pesaran (1999). The stochastic Solow growth model differs from its deterministic counterpart by explicitly allowing for random technological progress and random labor input in the otherwise deterministic model. The model has been empirically examined in, among others, Lee, Pesaran \& Smith (1997) and Pesaran (2007). The parameters of the log linearized solution for the stochastic Solow growth model can be estimated by a variety of panel data estimators discussed in Pesaran \& Smith (1995) and Pesaran (2006). The method proposed in Section 3 can also be adopted for this purpose. More standard empirical estimators, such as fixed effects, difference and system GMM estimators impose common slope parameters and can therefore not be considered for this model. They have been adopted for estimating variants of the deterministic Solow 
growth model with common slopes, see for example Islam (1995) and Caselli, Esquivel \& Lefort (1996).

We present an empirical study for a panel of economic growth rates for $N=34$ OECD countries for the years 1950 to 2010 that are listed in the Penn World Tables (PWT) version 7.1, see Heston, Summers \& Aten (2012) and Appendix A. The resulting panel of time series is highly unbalanced with many missing entries. We generalize the model considered by Lee et al. (1997) but replace the Gaussian density with the Student's $t$ density for the measurement errors. We remain to have heterogeneous stochastic growth trends and heterogeneous slope parameters in the model. The Student's $t$ density is included to capture large outliers. Further, it is likely that different countries have similar access to available technology. To capture these common paths, we model the stochastic trends by a multi-factor structure, see also Pesaran (2007) and Phillips \& Sul (2009).

Let $y_{i, t}=100\left(\log Y_{i, t}-\log Y_{i, t-1}\right)$, where $Y_{i, t}$ is the per capita output of country $i$ in year t. The model specification of Pesaran (2007) for the growth rates $y_{i, t}$ is given by

$$
y_{i, t}=\left(1-\gamma_{i}\right) g_{i}+\gamma_{i} y_{i, t-1}+(1-\alpha) \Delta \zeta_{i, t}+\left(\alpha-\gamma_{i}\right) \Delta \zeta_{i, t-1}-\alpha \Delta^{2} v_{i, t}
$$

where $\Delta$ is the difference operator, $g_{i}$ is the mean growth rate, $\gamma_{i}$ is the within-country convergence parameter, $\alpha$ is the elasticity of output with respect to capital and $\zeta_{i, t}$ and $v_{i, t}$ are the stochastic components of technology and employment, respectively. The model for $y_{i, t}$ is stationary and ergodic, regardless whether $\zeta_{i, t}$ or $v_{i, t}$ contain a unit root. The stochastic components of technology and employment cannot be empirically separated and are typically modeled by a country-specific autoregressive process. However, since common factors are likely to exists we can capture these terms by a multi-factor structure.

Model (25) can be expressed as a generalized dynamic panel data model, where the observations are modeled by the Student's $t$ density (C) in Table 1. We consider the signal $z_{i, t}$ in (2) with $w_{i, t}=0$ and $\epsilon_{i, t}$ as in (3). Further, $a_{i, t}=\left(1, y_{i, t-1}\right)^{\prime}$ such that the $2 \times 1$ vector $\mu_{i}$ captures the mean growth rate and the slope, $b_{i, t}=b_{i}$ is the $r \times 1$ vector of factor 
loadings and $\xi_{t}$ is the $r \times 1$ vector of common factors. The common factors follow independent autoregressive processes of order 2 and capture technology and employment shocks which may contain business cycle effects.

We estimate the coefficients of model specifications with Student's $t$ and Gaussian (fix $\nu=$ 1,000) densities. The most important parameter estimates for the Gaussian and Student's $t$ model are presented in Table 5 for the models with $r=2$ common factors. For $r=1, r=3$ and a variety of other model specifications similar results are obtained. When comparing the Student's $t$ with the Gaussian specification, we find that the Student's $t$ model yields a much higher loglikelihood value, both evaluated at their maximum likelihood estimates. The degrees of freedom $\nu$ is estimated as 2.41 which clearly indicates that many large and outlying shocks are present in the OECD PWT dataset.

The estimated mean growth rates $g_{i}$ and convergence parameters $\gamma_{i}$ are plotted per country in Figure 3. We find that the estimates for the Student's $t$ model are extracted with much more precision. The estimates for the Gaussian model correspond to the estimates reported by Lee et al. (1997) where the stochastic growth model is also considered for the levels of per capita output. The mean growth rates $g_{i}$ are positive and on average 2.47 for the Student's $t$ model and 2.44 for the Gaussian model. The implied speed of convergence $\gamma_{i}$ has mean around 0.3 for the Student's $t$ model and around 0.2 for the Gaussian model. Large differences in convergence rates exists for different countries.

In Figure 4 we present the estimated common trends $\xi_{t}$ and loadings $b_{i}$ for the Student's $t$ and Gaussian models. For the Gaussian model, the $95 \%$ confidence bounds for the estimates are much wider. For the Student's $t$ model a more pronounced business cycle emerges from the factor estimates. The first factor is normalized to the US growth rate. Each country loads positively on this factor. The second factor correspond to the western countries where economic growth has slowed down in the last decade. The large values found for the loadings for the Gaussian model can be explained by the large outliers in the growth rates. Examples are Estonia, Slovenia and Slovakia.

For all models considered in Sections 5.1, 5.2 and 5.3 we calculated the Wald test statistics 


\begin{tabular}{lrr} 
& Model 1: $y_{i, t} \sim t\left(z_{i, t}, \sigma_{\zeta}, \nu\right)$ & Model 2: $y_{i, t} \sim t\left(z_{i, t}, \sigma_{\zeta}, 1000\right)$ \\
\hline$\nu$ & $2.413_{0.227}$ & 1000 \\
$\sigma$ & $4.741_{2.025}$ & $2.788_{0.049}$ \\
$\delta_{1}$ & $1.680_{0.177}$ & $1.955_{0.235}$ \\
$\delta_{2}$ & $0.321_{0.030}$ & $0.200_{0.033}$ \\
$\sigma_{\mu, 1}$ & $0.291_{0.143}$ & $0.311_{0.130}$ \\
$\sigma_{\mu, 2}$ & $0.086_{0.020}$ & $0.118_{0.024}$ \\
$h_{1,1}$ & $0.102_{0.075}$ & $0.222_{0.165}$ \\
$h_{1,2}$ & $-0.005_{0.034}$ & $-0.012_{0.015}$ \\
$h_{2,1}$ & $0.236_{0.198}$ & $0.359_{0.149}$ \\
$h_{2,2}$ & $-0.052_{0.012}(y)$ & $-0.042_{0.014}$ \\
\hline $\log \hat{p}(y)$ & -7581.66 & -7807.39 \\
$\mathrm{AIC}$ & 15317.06 & 15768.52 \\
\hline
\end{tabular}

Table 5: Summary of the parameter estimation results (and standard errors in lower case) for the economic growth rate application. The model considered is given by observation density $(\mathrm{C})$ in Table 1 with the signal specification $z_{i, t}$ as discussed in Section 5.3 . The data panel consists of the growth rates for 34 countries between 1950 and 2010 listed in Appendix A.

for the importance sampling weights, see the discussion in Section 4.2. All test statistics are sufficiently negative, which indicates that a $\sqrt{M}$ convergence rate exists for all models. The individual results for the weight tests are presented in the Technical Appendix.

\section{Conclusion}

We have developed simulation-based methodology for the estimation of parameters in a general class of dynamic panel data models with cross-section and time-varying random effects. The new estimation method felicitates the modeling of high-dimensional non-Gaussian panel data. The use of importance sampling and related methods provides the means for a feasible analysis. The computational efficiency of our methods is due to the ability to separate the cross-section effects from the time-varying effects and to collapse high-dimensional vectors to low-dimensional vectors that contain the sufficient statistics relevant for the analysis. Further, the use of the Kalman filter allows for the efficient sampling of the time-varying effects. In the Monte Carlo study we have given clear evidence of the validity of our estimation methods for finite samples. The empirical illustrations highlight the relevance and flexibility of our methods. 

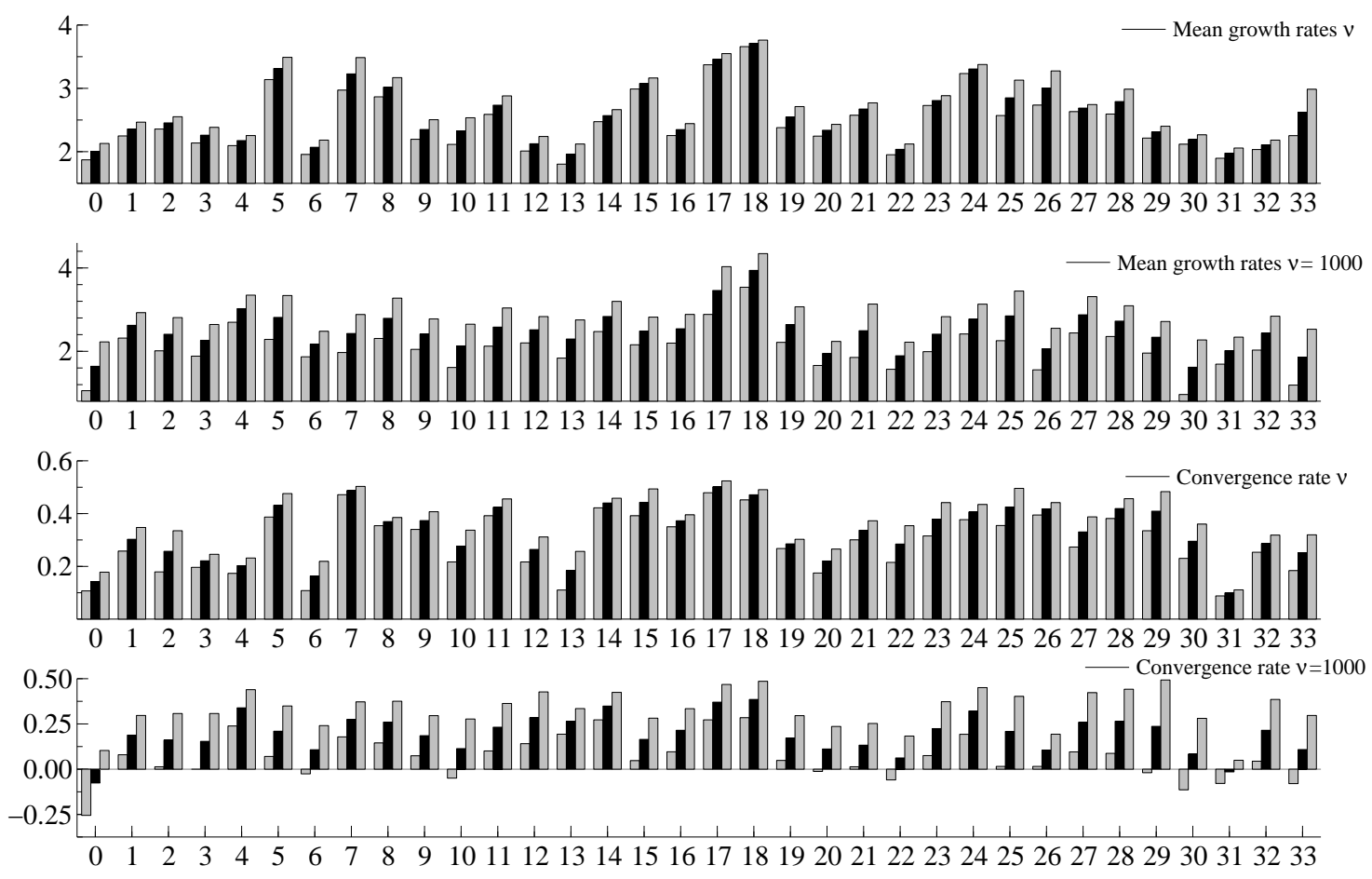

Figure 3: Country-specific effects for the economic growth model. The black bars indicate the estimated posterior means, whereas the lighter bars provide the confidence intervals.

\section{Appendix A}

Current OECD countries: 0. Australia (AUS), 1. Austria (AUT), 2. Belgium (BEL), 3. Canada (CAN), 4. Chile (CHL), 5. Czech Republic (CZE), 6. Denmark (DNK), 7. Estonia (EST), 8. Finland (FIN), 9. France (FRA), 10. Germany (DUE), 11. Greece (GRC), 12. Hungary (HUN), 13. Iceland (ISL), 14. Ireland (IRL), 15. Israel (ISR), 16. Italy (ITA), 17. Japan (JPN), 18. Korea (KOR), 19. Luxembourg (LUX), 20. Mexico (MEX), 21. Netherlands (NLD), 22. New Zealand (NLZ), 23. Norway (NOR), 24. Poland (POL), 25. Portugal (PRT), 26. Slovakia (SVK), 27. Slovenia (SVN), 28. Spain (ESP), 29. Sweden (SWE), 30. Switzerland (CHE), 31. Turkey (TUR), 32. United Kingdom (GBR), 33. United States (USA). 

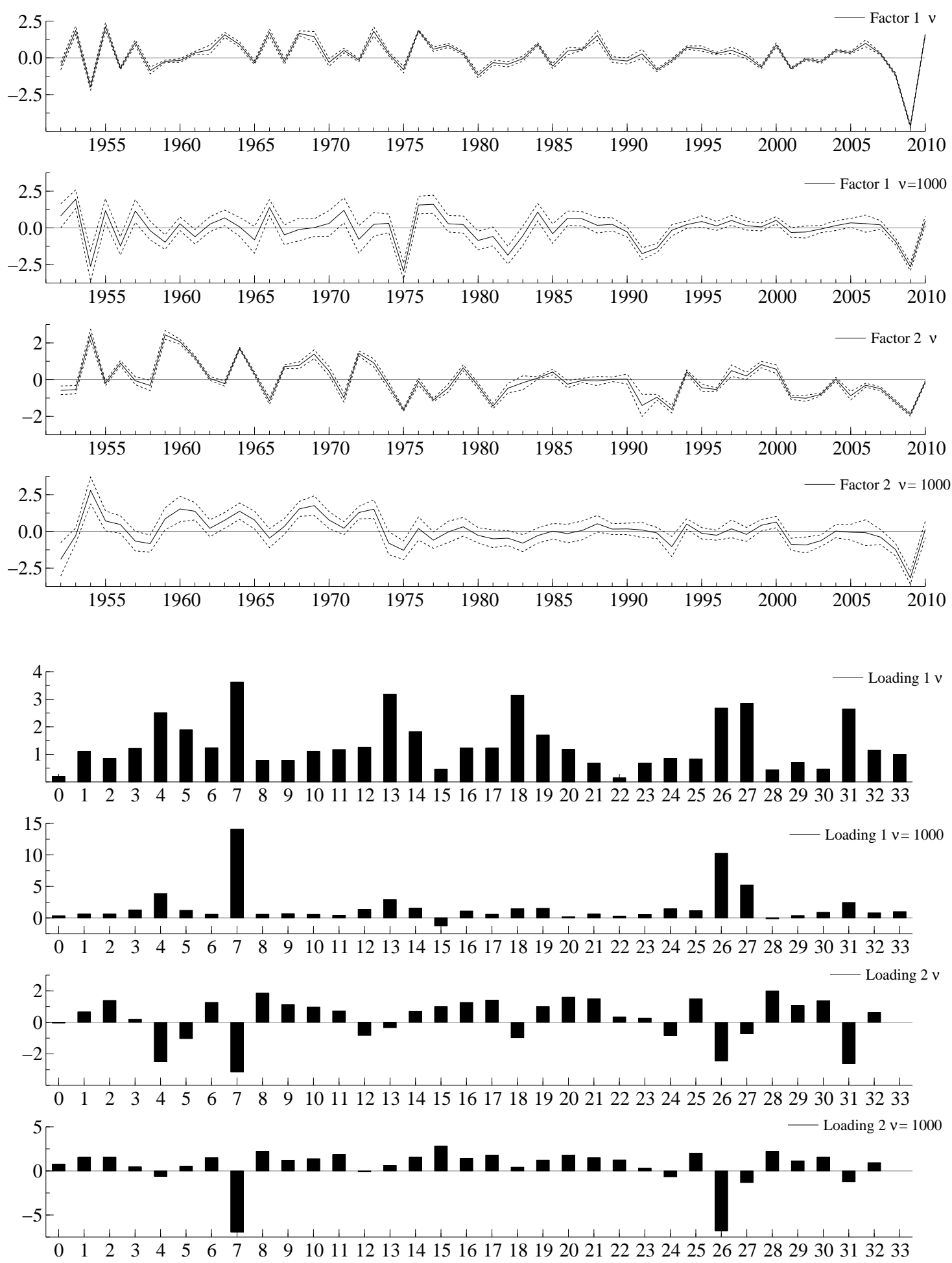

Figure 4: Top panel: Common time-varying factor estimates and $95 \%$ confidence bounds (dotted lines) for the Student's $t$ and Gaussian dynamic panel data models. Bottom panel: Factor loadings $b_{i}$ for the Student's $t$ and Gaussian dynamic panel data models. The effects are computed based on estimated parameters $\tilde{\psi}$ (given in Table 5 ). 


\section{References}

Arellano, M. \& Bond, S. (1991), 'Some Tests of Specification for Panel Data: Monte Carlo Evidence and an Application to Employment Equations', Review of Economic Studies 58, 277-297.

Arellano, M. \& Bonhomme, S. (2011), 'Nonlinear Panel Data Analysis', Annual Review of Economics 3, 395-424.

Baltagi, B. H. (2005), Econometric Analysis of Panel Data, John Wiley \& Sons, New York.

Bijleveld, C. C. J. H. \& Wijkman, M. (2009), 'Intergenerational continuity in convictions: A five-generation study', Criminal Behavior and Mental Health 19, 142-155.

Binder, M. \& Pesaran, M. H. (1999), 'Stochastic Growth Models and Their Econometric Implications', Journal of Economic Growth 4, 139-183.

Blundell, R. \& Bond, S. (1998), 'Initial Conditions and Moment Restrictions in Dynamic Panel Data Models', Journal of Econometrics 87, 115-143.

Caselli, F., Esquivel, G. \& Lefort, F. (1996), 'Reopening the Convergence Debate: A New Look at Cross-Country Growth Empirics', Journal of Economic Growth 1, 363-389.

Chamberlain, G. (1980), 'Analysis of Covariance with Qualitative Data', The Review of Economic Studies 47, 225-238.

Chib, S. (2008), Panel Data Modeling and Inference: A Bayesian Primer, in L. Mátyás \& P. Sevestre, eds, 'The Econometrics of Panel Data', Springer Verlag, Berlin Heidelberg, pp. $215-246$.

Doornik, J. A. (2007), Object-Oriented Matrix Programming Using Ox, Timberlake Consultants Press, London.

Duffie, D., Saita, L. \& Wang, K. (2007), 'Multi-period corporate default prediction with stochastic covariates', Journal of Financial Economics 83, 635-665. 
Durbin, J. \& Koopman, S. J. (1997), 'Monte Carlo maximum likelihood estimation of nonGaussian state space models', Biometrika 84, 669-684.

Durbin, J. \& Koopman, S. J. (2000), 'Time series analysis of non-Gaussian observations based on state space models from both classical and Bayesian perspectives', Journal of the Royal Statistical Society, Series B 62, 3-56.

Durbin, J. \& Koopman, S. J. (2002), 'A simple and efficient simulation smoother for state space time series analysis', Biometrika 89, 603-616.

Durbin, J. \& Koopman, S. J. (2012), Time Series Analysis by State Space Methods, 2 edn, Oxford University Press, Oxford.

Durlauf, S. N., Johnson, P. \& Temple, J. (2005), Growth Econometrics, in P. Aghion \& S. N. Durlauf, eds, 'Handbook of Economic Growth, vol. 1, Part A,', Elsevier, Amsterdam, North-Holland, pp. 555-677.

Geweke, J. F. (1989), 'Bayesian Inference in Econometric Models Using Monte Carlo Integration', Econometrica 57, 1317-1339.

Geweke, J. F. (1991), 'Efficient Simulation from the Multivariate Normal and Student-t Distributions Subject to Linear Constraints', Computer Science and Statistics. Proceedings of the 23rd Symposium on the Interface. Seattle Washington, April 21-24, 1991 pp. 571578.

Geweke, J. \& Keane, M. (2001), Computationally Intensive Methods for Integration in Econometrics, in J. J. Heckman \& E. Leamer, eds, 'Handbook of Econometrics, V', Elsevier, Amsterdam, North-Holland, pp. 3465-3568.

Hajivassiliou, V. (1990), 'Smooth Simulation Estimation of Panel Data LDV Models'. Mimeo, Yale University.

Harvey, A. C. (1989), Forecasting, Structural Time Series Models and the Kalman Filter, Cambridge University Press, Cambridge. 
Heckman, J. J. (1981a), Heterogeneity and State Dependence, in S. Rosen, ed., 'Studies of Labor Markets', The University of Chicago Press, Chicago: The National Bureau of Economic Research, pp. 91-140.

Heckman, J. J. (1981b), Statistical Models for Discrete Panel Data, in C. F. Manski \& D. L. McFadden, eds, 'Structural Analysis of Discrete Data and Econometric Applications', The MIT Press, Cambridge, pp. 113-177.

Heiss, F. (2008), 'Sequential Numerical Integration in Nonlinear State Space Models for Microeconometric Panel Data', Journal of Applied Econometrics 23, 373-389.

Heston, A., Summers, R. \& Aten, B. (2012), 'Penn world table version 7.1'. Center for International Comparisons of Production, Income and Prices at the University of Pennsylvania.

Hsiao, C. (2003), Analysis of Panel Data, Cambridge University Press, Cambridge.

Hsiao, C. \& Pesaran, M. H. (2008), Random Coefficients Models, in L. Mátyás \& P. Sevestre, eds, 'The Econometrics of Panel Data', Springer Verlag, Berlin Heidelberg, pp. 187-216.

Islam, N. (1995), 'Growth Empirics: A Panel Data Approach', The Quarterly Journal of Economics 110, 1127-1170.

Jungbacker, B. \& Koopman, S. J. (2007), 'Monte Carlo Estimation for Nonlinear NonGaussian State Space Models', Biometrika 94, 827-839.

Jungbacker, B. \& Koopman, S. J. (2008), 'Likelihood-based Analysis for Dynamic Factor Models'. Tinbergen Institute Discussion Paper: 08-007/4.

Jungbacker, B., Koopman, S. J. \& van der Wel, M. (2011), 'Maximum likelihood estimation for dynamic factor models with missing data', Journal of Economic Dynamics and Control 35, 1358-1368. 
Keane, M. P. (1994), 'A Computationally Practical Simulation Estimator for Panel Data', Econometrica 62, 95-116.

Koopman, S. J. \& Lucas, A. (2008), 'A Non-Gaussian Panel Time Series Model for Estimating and Decomposing Default Risk', Journal of Business and Economic Statistics 26, $510-525$.

Koopman, S. J., Ooms, M., Lucas, A., Montfort, K. \& Van der Geest, V. (2008), 'Estimating systematic continuous-time trends in recidivism using a non-Gaussian panel data model', Statistica Neerlandica 62, 104-130.

Koopman, S. J., Shephard, N. \& Creal, D. D. (2009), 'Testing the assumptions behind importance sampling', Journal of Econometrics 149, 2-11.

Lee, K., Pesaran, H. M. \& Smith, R. (1997), 'Growth and Convergence in a Multi-Country Empirical Stochastic Solow Model', Journal of Applied Econometrics 12, 357-392.

Lee, L. F. (1997), 'Simulated maximum likelihood estimation of dynamic discrete choice models, Some Monte Carlo results', Journal of Econometrics 82, 1-35.

Liesenfeld, R., Moura, G. V. \& Richard, J. F. (2010), 'Determinants and Dynamics of Current Account Reversals: An Empirical Analysis', Oxford Bulletin of Economics and Statistics 72, 486-517.

Liesenfeld, R. \& Richard, J. (2008), Simulation Techniques for Panels: Efficient Sampling, in L. Mátyás \& P. Sevestre, eds, 'The Econometrics of Panel Data', Springer Verlag, Berlin Heidelberg, pp. 215-246.

Liesenfeld, R. \& Richard, J. F. (2010), 'Efficient Estimation of Probit Modeld with Correlated Errors', Journal of Econometrics 2010, 367-376.

Monahan, J. F. (2001), Numerical Methods of Statistics, Cambridge University Press, Cambridge. 
Nocedal, J. \& Wright, S. J. (1999), Numerical Optimization, Springer Verlag, New York.

Pesaran, H. M. (2007), 'A Pair-Wise Approach To Testing For Output And Growth Convergence', Journal of Econometrics 138, 312-355.

Pesaran, M. H. (2006), 'Estimation and Inference in Large Heterogeneous Panels with a Multifactor Error Structure', Econometrica 74, 967-1012.

Pesaran, M. H. \& Smith, R. (1995), 'Estimating long-run relationships from dynamic heterogeneous panels', Journal of Econometrics 68, 79-113.

Phillips, P. C. B. \& Sul, D. (2009), 'Economic Transition and Growth', Journal of Applied Econometrics 24, 1153-1185.

Richard, J. F. \& Zhang, W. (2007), 'Efficient High-Dimensional Importance Sampling', Journal of Econometrics 141, 1385-1411.

Ripley, B. D. (1987), Stochastic Simulation, John Wiley \& Sons, New York.

Shephard, N. \& Pitt, M. K. (1997), 'Likelihood analysis of non-Gaussian measurement time series', Biometrika 84, 653-667.

So, M. K. P. (2003), 'Posterior mode estimation for nonlinear and non-Gaussian state space models', Statistica Sinica 13, 255-274.

Stock, J. H. \& Watson, M. W. (2002), 'Macroeconomic Forecasting Using Diffusion Indexes', Journal of Business and Economic Statistics 220, 147-162.

Vella, F. \& Verbeek, M. (1998), 'Whose Wages do Unions Raise? A Dynamic Model of Unionism and Wage Rate Determination for Young Men', Journal of Applied Econometrics 13, 163-183.

Wooldridge, J. M. (2005), 'Simple solutions to the initial conditions problem in dynamic, nonlinear panel data models with unobserved heterogeneity', Journal of Applied Econometrics 20, 39-54. 


\title{
Technical Appendix for: Generalized Dynamic Panel Data Models with Random Effects for Cross-Section and Time
}

\author{
G. Mesters ${ }^{(a, b) *}$ and S.J. Koopman ${ }^{(b, c)}$ \\ (a) Netherlands Institute for the Study of Crime and Law Enforcement, \\ (b) Department of Econometrics, VU University Amsterdam, \\ (c) Tinbergen Institute, Amsterdam
}

January 28, 2014

\begin{abstract}
In this technical appendix we present the details for the likelihood evaluation procedure of Mesters \& Koopman (2014, Section 3) and the estimation of the posterior random effects. Further, we present the complete set of simulation and empirical results that are discussed in Mesters \& Koopman (2014).
\end{abstract}

\section{Details Likelihood Evaluation}

The likelihood estimate $\hat{p}(y)$ for the generalized dynamic panel data model (Mesters \& Koopman (2014, Section 2)) is given in Mesters \& Koopman (2014, equation 10) by

$$
\hat{p}(y)=g(y ; \hat{\xi}) g(y ; \hat{\mu}) \frac{1}{M} \sum_{i=1}^{M} w^{(i)},
$$

where the weights are

$$
w^{(i)}=p\left(y \mid \mu^{(i)}, \xi^{(i)} ; x\right) /\left[g\left(y \mid \xi^{(i)} ; \hat{\mu}\right) g\left(y \mid \mu^{(i)} ; \hat{\xi}\right)\right] .
$$

${ }^{*}$ Corresponding author: G. Mesters, PhD candidate Department of Econometrics, VU University Amsterdam, The Netherlands. Contact address: NSCR, PO box 71304, 1008 BH Amsterdam, The Netherlands, tel. nr. +31 2059 89405, email: gmesters@nscr.nl, Technical Appendix: http://www.geertmesters.nl and http://personal.vu.nl/s.j.koopman 
The samples $\left\{\mu^{(1)}, \ldots, \mu^{(M)}\right\}$ are drawn independently from importance density $g(\mu \mid y ; \hat{\xi})$ and samples $\left\{\xi^{(1)}, \ldots, \xi^{(M)}\right\}$ from $g(\xi \mid y ; \hat{\mu})$. We now discuss in detail how to construct these importance densities.

We choose both densities to follow Gaussian distributions and modify their means and variances such that their modes are equal to the modes of the original posterior density $p(\mu, \xi \mid y ; x)$. Similar strategies are followed for models without random individual-specific effects; see for example, Shephard \& Pitt (1997) and Durbin \& Koopman (1997, 2000). So (2003) and Jungbacker \& Koopman (2007) argue that this strategy can be implemented by numerically maximizing $\log p(\mu, \xi \mid y ; x)=\log p(y \mid \mu, \xi ; x)+\log p(\mu, \xi)-\log p(y ; x)$ with respect to $\mu$ and $\xi$.

The instrumental basis to facilitate this numerical maximization is given, for variable $y_{i, t}$, by the linear Gaussian panel data model

$$
y_{i, t}=c_{i, t}+\epsilon_{i, t}+u_{i, t}, \quad u_{i, t} \sim \operatorname{NID}\left(0, d_{i, t}^{2}\right)
$$

where $c_{i, t}$ is a fixed constant, stochastic component $\epsilon_{i, t}$ is given by Mesters \& Koopman (2014, equation 3) and $u_{i, t}$ is a random variable with mean zero and fixed variance $d_{i, t}^{2}$. The stochastic component $\epsilon_{i, t}$ is the same as in the original model of interest. The predetermined component $w_{i, t}$ is not explicitly included in approximating model (3) since it is fixed at time $t$. The constants $c_{i, t}$ and $d_{i, t}$ are chosen such that (3) can be used to compute the posterior modal values $\hat{\mu}$ and $\hat{\xi}$, respectively. The elements $u_{i, t}$ and $\epsilon_{j, s}$ are uncorrelated with each other, for all $i, j=1, \ldots, N$ and $s, t=1, \ldots, T$. Furthermore, $u_{i, t}$ is serially uncorrelated. It follows that

$$
g(y \mid \mu, \xi)=\prod_{i=1}^{N} \prod_{t=1}^{T} g\left(y_{i, t} \mid \mu_{i}, \xi_{t}\right), \quad \text { with } \quad g\left(y_{i, t} \mid \mu_{i}, \xi_{t}\right) \equiv \operatorname{NID}\left(c_{i, t}+\epsilon_{i, t}, d_{i, t}^{2}\right) .
$$

The maximization of $\log p(\mu, \xi \mid y ; x)$ with respect to $\mu$ and $\xi$ can be carried out via the Newton-Raphson method. The idea is to iterate between linearizing $p(y \mid \mu, \xi ; x)$, by computing $c=\left\{c_{i, t}\right\}$ and $d=\left\{d_{i, t}\right\}$, to obtain $g(y \mid \mu, \xi)$ and updating $\mu$ and $\xi$ based on the linearized model given by equations (3) and Mesters \& Koopman (2014, equation 3). The following algorithm summarizes this method.

\section{Algorithm A}

(i) Initialize the algorithm by choosing $\mu^{*}$ and $\xi^{*}$ as starting values, which gives $\epsilon_{i, t}^{*}$ and $z_{i, t}^{*}$, for all $i=1, \ldots, N$ and $t=1, \ldots, T$;

(ii) Given the set of two equations

$$
\frac{\partial \log p\left(y_{i, t} \mid z_{i, t}\right)}{\partial z_{i, t}}=\frac{\partial \log g\left(y_{i, t} \mid \epsilon_{i, t}\right)}{\partial \epsilon_{i, t}}, \quad \frac{\partial^{2} \log p\left(y_{i, t} \mid z_{i, t}\right)}{\partial z_{i, t} \partial z_{i, t}}=\frac{\partial^{2} \log g\left(y_{i, t} \mid \epsilon_{i, t}\right)}{\partial \epsilon_{i, t} \partial \epsilon_{i, t}}
$$

for $i=1, \ldots, N$ and $t=1, \ldots, T$, where $p\left(y_{i, t} \mid z_{i, t}\right)$ is the observation model Mesters \& Koopman (2014, equation 5) and $g\left(y_{i, t} \mid \epsilon_{i, t}\right)$ is given by (4), we can deduct expressions 
for $c_{i, t}$ and $d_{i, t}$ as functions of $z_{i, t}$, and compute $c_{i, t}=c_{i, t}^{*}$ and $d_{i, t}=d_{i, t}^{*}$ for $\epsilon_{i, t}=\epsilon_{i, t}^{*}$ and $z_{i, t}=z_{i, t}^{*}$;

(iii) Compute $\tilde{\mu}=\mathrm{E}_{g}\left(\mu \mid y ; \xi^{*}\right)$ from the resulting model $(3)$ with $\xi=\xi^{*}, c_{i, t}=c_{i, t}^{*}$ and $d_{i, t}=d_{i, t}^{*}$

(iv) Replace $\mu^{*}$ by $\mu^{*}=\tilde{\mu}$;

(v) Compute $\tilde{\xi}=\mathrm{E}_{g}\left(\xi \mid y ; \mu^{*}\right)$ from the resulting model $(3)$ with $\mu=\mu^{*}, c_{i, t}=c_{i, t}^{*}$ and $d_{i, t}=d_{i, t}^{*}$

(vi) Replace $\xi^{*}$ by $\xi^{*}=\tilde{\xi}$

(vii) Iterate from (ii) to (vi) until convergence.

Since the mode and the mean of the approximating linear Gaussian model are set equal to the mode of the original model, it holds that $\tilde{\mu}=\hat{\mu}=\operatorname{argmax}_{\mu} p(\mu \mid y ; \hat{\xi} ; x)$ and $\tilde{\xi}=\hat{\xi}=$ $\operatorname{argmax}_{\mu} p(\xi \mid y ; \hat{\mu} ; x)$. Further, it holds that $\{\hat{\mu}, \hat{\xi}\}=\operatorname{argmax}_{\mu, \xi} p(\mu, \xi \mid y ; x)$.

The performance of Algorithm A depends crucially on the efficient computation of the conditional expectations in steps (iii) and (v). With respect to step (iii), for a given value of $\xi^{*}$, the approximating model (3) is reduced to a standard random effects model, with weighted individual-specific effects and heteroskedastic error term $u_{i, t}$, see Baltagi $(2005$, Chapters 2 and 5). This simplification becomes clear when we concatenate the observations $y_{i, t}$ over the time index $t$ and when we consider the approximating model (3). We then obtain

$$
\bar{y}_{i}=\bar{c}_{i}+\bar{A}_{i} \mu_{i}+\overline{\mathcal{B}}_{i}+\bar{u}_{i}, \quad \bar{u}_{i} \sim \operatorname{NID}\left(0, \bar{D}_{i}\right), \quad i=1, \ldots, N
$$

where $\bar{y}_{i}=\left(y_{i, 1}, \ldots, y_{i, T}\right)^{\prime}, \bar{c}_{i}=\left(c_{i, 1}, \ldots, c_{i, T}\right)^{\prime}, \bar{A}_{i}=\left(a_{i, 1}, \ldots, a_{i, T}\right)^{\prime}, \overline{\mathcal{B}}_{i}=\left(b_{i, 1}^{\prime} \xi_{1}, \ldots, b_{i, T}^{\prime} \xi_{T}\right)^{\prime}$ and $\bar{u}_{i}=\left(u_{i, 1}, \ldots, u_{i, T}\right)^{\prime}$. The $T \times T$ variance matrix $\bar{D}_{i}$ is diagonal by construction, with elements $d_{i, 1}^{2}, \ldots, d_{i, T}^{2}$ on the main diagonal. Based on (5), the computation of $\mathrm{E}_{g}\left(\mu \mid y ; \xi^{*}\right)$ can be performed using standard multivariate normal regression theory. In particular, it holds that

$$
\mathrm{E}_{g}\left(\mu \mid y ; \xi^{*}\right)=\mathrm{E}_{g}\left(\mu ; \xi^{*}\right)+\operatorname{Cov}_{g}\left(\mu, y ; \xi^{*}\right) \operatorname{Var}_{g}\left(y ; \xi^{*}\right)^{-1}\left[y-\mathrm{E}_{g}\left(y ; \xi^{*}\right)\right]
$$

which can be solved separately for each element $\mathrm{E}_{g}\left(\mu_{i} \mid \bar{y}_{i} ; \xi^{*}\right)$, as given $\xi^{*}, \mu_{i}$ only depends on $y$ by means of $\bar{y}_{i}$. Some simple manipulations give

- $\mathrm{E}_{g}\left(\mu_{i} ; \xi^{*}\right)=\delta$;

- $\operatorname{Cov}_{g}\left(\mu_{i}, \bar{y}_{i} ; \xi^{*}\right)=\Sigma_{\mu} \bar{A}_{i}^{\prime}$;

- $\operatorname{Var}_{g}\left(\bar{y}_{i} ; \xi^{*}\right)^{-1}=\bar{D}_{i}^{-1}-\bar{D}_{i}^{-1} \bar{L}_{i}\left(\bar{L}_{i}^{\prime} \bar{D}_{i}^{-1} \bar{L}_{i}+I_{q}\right)^{-1} \bar{L}_{i}^{\prime} \bar{D}_{i}^{-1}$, where $\bar{L}_{i}=\bar{A}_{i} \cdot$ choleski $\left(\Sigma_{\mu}\right)$, see Roy \& Sarhan (1956) and Roy (1958);

- $\mathrm{E}_{g}\left(\bar{y}_{i} ; \xi^{*}\right)=\bar{c}_{i}+\overline{\mathcal{B}}_{i}^{*}+\bar{A}_{i} \delta$. 
Efficient implementation of the calculated can be accomplished without storing variance matrices $\operatorname{Var}_{g}\left(\bar{y}_{i} ; \xi^{*}\right)$ or its inverses.

Now consider step (v) where we need to compute $\mathrm{E}_{g}\left(\xi \mid y ; \mu^{*}\right)$. Given a value of $\mu^{*}$, approximating model (3), can be written as a linear Gaussian state space model. This can be seen by concatenating variables $y_{i, t}$ over the cross-section dimension, which gives

$$
y_{t}=c_{t}+\mathcal{A}_{t}+B_{t} \xi_{t}+u_{t}, \quad u_{t} \sim \operatorname{NID}\left(0, D_{t}\right), \quad t=1, \ldots, T
$$

where $y_{t}=\left(y_{1, t}, \ldots, y_{N, t}\right)^{\prime}, c_{t}=\left(c_{1, t}, \ldots, c_{N, t}\right)^{\prime}, \mathcal{A}_{t}=\left(a_{1, t}^{\prime} \mu_{1}, \ldots, a_{N, t}^{\prime} \mu_{N}\right)^{\prime}, B_{t}=\left(b_{1, t}, \ldots, b_{N, t}\right)^{\prime}$ and $u_{t}=\left(u_{1, t}, \ldots, u_{N, t}\right)^{\prime}$. Variance matrix $D_{t}$ is diagonal by construction, with elements $d_{1, t}^{2}, \ldots, d_{N, t}^{2}$ on the main diagonal. Based on (7) the computation of $\mathrm{E}_{g}\left(\xi \mid y ; \mu^{*}\right)$ is carried out using the Kalman filter and smoothing methods; see Anderson \& Moore (1979) and Durbin \& Koopman (2012, Chapter 4). Moreover, since $D_{t}$ is diagonal the fast Kalman filter and smoothing methods from Koopman \& Durbin (2003) can be used.

The evaluation of likelihood estimate $\hat{p}(y)$ in (1), requires $M$ samples of $\mu$ and $\xi$ from importance densities $g(\mu \mid y ; \hat{\xi})$ and $g(\xi \mid y ; \hat{\mu})$, respectively. The posterior modal values $\hat{\mu}$ and $\hat{\xi}$ are obtained from Algorithm A. Both importance densities are based on approximating model (3). The vector representations (5) and (7), are adopted for computing the $M$ samples by using the simulation smoother methods of Durbin \& Koopman (2002). However, both representations have large dimensions leading to simulation smoother methods that are computationally demanding. Instead, we show that more efficiency can be obtained by first performing two transformations to reduce the cross-section and time series dimensions of observed data $y$. In particular, the vectors series $\bar{y}_{i}$ and $y_{t}$ in equations (5) and (7), can be transformed into two low-dimensional vector series $\bar{y}_{i}^{l}$ and $y_{t}^{l}$, for $t=1, \ldots, T$ and $i=1, \ldots, N$. Based on these vector series, samples $\xi^{(i)}$ and $\mu^{(i)}$ can be drawn from $g\left(\xi \mid y^{l} ; \hat{\mu}\right)$ and $g\left(\mu \mid \bar{y}^{l} ; \hat{\xi}\right)$, respectively, where $\bar{y}^{l}=\left[\left(\bar{y}_{1}^{l}\right)^{\prime}, \ldots,\left(\bar{y}_{N}^{l}\right)^{\prime}\right]^{\prime}$ and $y^{l}=\left[\left(y_{1}^{l}\right)^{\prime}, \ldots,\left(y_{T}^{l}\right)^{\prime}\right]^{\prime}$. The resulting samples can be regarded as coming from $g(\mu \mid y ; \hat{\xi})$ and $g(\xi \mid y ; \hat{\mu})$, respectively. In Section 2 we present the computational gains in evaluating the likelihood, for both sets of importance densities. The computational improvements resulting from the transformations are high. Apart from the computational gains, we also need to use less common random numbers for sampling the same number of draws $\mu^{(i)}$ and $\xi^{(i)}$, regardless of the simulation smoother used.

For the simulation of time-varying effects $\xi^{(i)}$ from $g\left(\xi \mid y^{l} ; \hat{\mu}\right)$, we collapse $N \times 1$ vectors $y_{t}$, based on equation (7), into low-dimensional vectors $y_{t}^{l}$, without losing information relevant for the extraction of $\xi$. This transformation has been introduced in Jungbacker \& Koopman (2008) for the efficient evaluation of the likelihood for linear Gaussian dynamic factor models. Here only mild modifications of their methods are required.

Consider a linear approximating model for transformed data $y_{t}^{*}=S_{t}\left(y_{t}-c_{t}-\hat{\mathcal{A}}_{t}\right)$ where $S_{t}$ is an $N \times N$ nonsingular projection matrix and where $y_{t}, c_{t}$ and $\mathcal{A}_{t}$ are as given by (7) with $\mathcal{A}_{t}$ replaced by $\hat{\mathcal{A}}_{t}=\left(a_{1, t}^{\prime} \hat{\mu}_{1}, \ldots, a_{N, t}^{\prime} \hat{\mu}_{N}\right)^{\prime}$ and $\hat{\mu}_{i}$ is the vector of posterior modal individual-specific effects for time series $i$, for $i=1, \ldots, N$ and $t=1, \ldots, T$. The transformed 
observations are given by

$$
y_{t}^{*}=\left[\begin{array}{c}
y_{t}^{l} \\
y_{t}^{h}
\end{array}\right], \quad \text { with } \begin{array}{r}
y_{t}^{l}=S_{t}^{l}\left(y_{t}-c_{t}-\hat{\mathcal{A}}_{t}\right) \\
y_{t}^{h}=S_{t}^{h}\left(y_{t}-c_{t}-\hat{\mathcal{A}}_{t}\right)
\end{array}, \quad S_{t}=\left[\begin{array}{c}
S_{t}^{l} \\
S_{t}^{h}
\end{array}\right], \quad t=1, \ldots, T,
$$

where the partioned projection matrices $S_{t}^{l}$ and $S_{t}^{h}$ have dimensions $r \times N$ and $(N-r) \times N$, respectively. As a result the observation vectors $y_{t}^{l}$ and $y_{t}^{h}$ become of dimensions $r \times 1$ and $(N-r) \times 1$. We aim to choose $S_{t}^{l}$ and $S_{t}^{h}$ such that $y_{t}^{l}$ and $y_{t}^{h}$ are uncorrelated and only $y_{t}^{l}$ depends on $\xi_{t}$. In particular, we aim for a model of the form

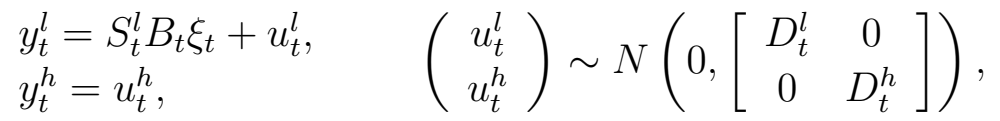

where $D_{t}^{l}=S_{t}^{l} D_{t} S_{t}^{l^{\prime}}$ and $D_{t}^{h}=S_{t}^{h} D_{t} S_{t}^{h^{\prime}}$ are $r \times r$ and $(N-r) \times(N-r)$ variance matrices, respectively.

Suitable matrices $S_{t}$, which lead to model (9) need to satisfy the following conditions; (a) matrices $S_{t}$ needs to be of full rank to prevent the loss of information, (b) $S_{t}^{h} D_{t} S_{t}^{l^{\prime}}=0$ to ensure that observations $y_{t}^{l}$ and $y_{t}^{h}$ are independent, and (c) $S_{t}^{h} B_{t} \xi_{t}=0$ to ensure that $y_{t}^{h}$ does not depend on $\xi_{t}$. Many matrix series $S_{t}$ can be found that fulfill these conditions. A convenient choice is given by

$$
S_{t}^{l}=\Delta_{t}^{\prime} B_{t}^{\prime} D_{t}^{-1}, \quad \Delta_{t} \Delta_{t}^{\prime}=\left(B_{t}^{\prime} D_{t}^{-1} B_{t}\right)^{-1},
$$

with $\Delta_{t}$ being a lower triangular matrix. This choice for $S_{t}^{l}$ results in

$$
y_{t}^{l}=\Delta_{t}^{-1} \xi_{t}+u_{t}^{l}, \quad u_{t}^{l} \sim \operatorname{NID}\left(0, I_{r}\right), \quad t=1, \ldots, T
$$

where $\Delta_{t}^{-1}$ is a $r \times r$ lower triangular matrix, $\xi_{t}$ is defined in Mesters \& Koopman (2014, equation 4 ) and $u_{t}^{l}$ is a random vector with mean zero and variance equal to the $r$-dimensional unit matrix $I_{r}$. Sampling time-varying effects $\xi^{(i)}$ from $g\left(\xi \mid y^{l} ; \hat{\mu}\right)$ is performed by applying the simulation smoother methods of Durbin \& Koopman (2002) to $r$-dimensional vector series $y_{t}^{l}$ and model (11), for $t=1, \ldots, T$. The matrices $S_{t}^{h}$ remain of large dimensions and can be constructed from $S_{t}^{l}$ but they are not required for any of the necessary computations. Further discussions of this transformation method are given in Jungbacker \& Koopman (2008)

For the simulation of individual-specific effects $\mu^{(i)}$ from $g\left(\mu \mid \bar{y}^{l} ; \hat{\xi}\right)$ we collapse $T \times 1$ vectors $\bar{y}_{i}$, for $i=1, \ldots, N$, based on vector representation $(5)$, with $\overline{\mathcal{B}}_{i}$ replaced by $\hat{\mathcal{B}}_{i}=$ $\left(b_{i, 1}^{\prime} \hat{\xi}_{1}, \ldots, b_{i, T}^{\prime} \hat{\xi}_{T}\right)^{\prime}$. We consider similar least squares type transformations as for the crosssection dimension above. However, because $\mu_{i}$ and $\mu_{j}$ are independent, the transformed observations $\bar{y}_{i}^{*}$ become simple rescaled averages of the variables in $\bar{y}_{i}$. Let

$$
\bar{y}_{i}^{*}=\left[\begin{array}{c}
\bar{y}_{i}^{l} \\
\bar{y}_{i}^{h}
\end{array}\right], \quad \text { with } \quad \begin{aligned}
\bar{y}_{i}^{l} & =\bar{S}_{i}^{l}\left(\bar{y}_{i}-\bar{c}_{i}-\hat{\mathcal{B}}_{i}\right) \\
\bar{y}_{i}^{h} & =\bar{S}_{i}^{h}\left(\bar{y}_{i}-\bar{c}_{i}-\hat{\mathcal{B}}_{i}\right)
\end{aligned}, \quad i=1, \ldots, N .
$$

The motivation of the transformation is the same as above. We require to sample $\mu_{i}$ based 
on only $\bar{y}_{i}^{l}$ without compromising data information. We choose matrices $\bar{S}_{i}^{l}$ and $\bar{S}_{i}^{h}$ to have dimensions $q \times T$ and $(T-q) \times T$, respectively. The model we aim to construct is given by

$$
\begin{aligned}
& \bar{y}_{i}^{l}=\bar{S}_{i}^{l} \bar{A}_{i} \mu_{i}+\bar{u}_{i}^{l}, \quad\left(\begin{array}{c}
\bar{u}_{i}^{l} \\
\bar{u}_{i}^{h}
\end{array}\right) \sim N\left(0,\left[\begin{array}{cc}
\bar{D}_{i}^{l} & 0 \\
0 & \bar{D}_{i}^{h}
\end{array}\right]\right),
\end{aligned}
$$

where $\bar{D}_{i}^{l}=\bar{S}_{i}^{l} \bar{D}_{i} \bar{S}_{i}^{l^{\prime}}$ and $\bar{D}_{i}^{h}=\bar{S}_{i}^{h} \bar{D}_{i} \bar{S}_{i}^{h^{\prime}}$ are $q \times q$ and $(N-q) \times(N-q)$ variance matrices respectively. A convenient choice for $\bar{S}_{i}^{l}$, which satisfies the conditions stated above, is given by

$$
\bar{S}_{i}^{l}=\bar{\Delta}_{i}^{\prime} \bar{A}_{i}^{\prime} \bar{D}_{i}^{-1}, \quad \bar{\Delta}_{i} \bar{\Delta}_{i}^{\prime}=\left(\bar{A}_{i}^{\prime} \bar{D}_{i}^{-1} \bar{A}_{i}\right)^{-1}, \quad .
$$

with $\bar{\Delta}_{i}$ being a lower triangular matrix. The resulting model for $\bar{y}_{i}^{l}$ is given by

$$
\bar{y}_{i}^{l}=\bar{\Delta}_{i}^{-1} \mu_{i}+\bar{u}_{i}^{l}, \quad \bar{u}_{t}^{l} \sim \operatorname{NID}\left(0, I_{q}\right), \quad i=1, \ldots, N,
$$

where $\bar{\Delta}_{i}^{-1}$ is a lower triangular $q \times q$ matrix, $\mu_{i}$ is given in Mesters \& Koopman (2014, equation 4) and $\bar{u}_{i}^{l}$ is a random vector with mean zero and $q \times q$ unit variance. Again we can construct large matrices $\bar{S}_{i}^{h}$, but they are not required for any necessary computations. Samples $\mu^{(i)}$ can be drawn independently from $g\left(\mu_{i} \mid \bar{y}_{i}^{l} ; \hat{\xi}\right)$, which is a Gaussian density with mean $\Sigma_{\mu} \bar{\Delta}_{i}^{-1}\left(\bar{\Delta}_{i}^{-1^{\prime}} \Sigma_{\mu} \bar{\Delta}_{i}^{-1}+I_{q}\right)^{-1} \bar{y}_{i}^{l}$ and variance $\Sigma_{\mu}-\Sigma_{\mu} \bar{\Delta}_{i}^{-1}\left(\bar{\Delta}_{i}^{-1^{\prime}} \Sigma_{\mu} \bar{\Delta}_{i}^{-1}+I_{q}\right)^{-1} \bar{\Delta}_{i}^{-1^{\prime}} \Sigma_{\mu}$. Both expressions follow from the standard lemma (6).

Next we discuss the construction of the Monte Carlo likelihood estimate $\hat{p}(y)$ in equation (1). The estimate relies on densities $g(y ; \hat{\mu})$ and $g(y ; \hat{\xi})$, that are based on the approximating model (3). Density $\log g(y ; \hat{\mu})$ can be computed from the prediction error decomposition of vector representation (7), with $\mu$ replaced by $\hat{\mu}$. This is obtained by a single pass through the Kalman filter, see Durbin \& Koopman (2012, Chapter 7). Computational efficiency can increased by using the lower dimensional model (11), based on vector series $y_{t}^{l}$. In particular, Jungbacker \& Koopman (2008) show that

$$
\log g(y ; \hat{\mu})=\text { constant }+\log g\left(y^{l} ; \hat{\mu}\right)-\frac{1}{2} \sum_{t=1}^{T} \log \left|D_{t}\right|+e_{t}^{\prime} D_{t}^{-1} e_{t}
$$

where $y^{l}=\left(y_{1}^{l^{\prime}}, \ldots, y_{T}^{l^{\prime}}\right)^{\prime}$ and $e_{t}=y_{t}-c_{t}-\hat{\mathcal{A}}_{t}-B_{t}\left(B_{t}^{\prime} D_{t}^{-1} B_{t}\right)^{-1} B_{t}^{\prime} D_{t}^{-1}\left(y_{t}-c_{t}-\hat{\mathcal{A}}_{t}\right)$ is the generalized least squares residual vector. Density $g\left(y^{l} ; \hat{\mu}\right)$ can be computed from the prediction error decomposition of model (11), which is a $r \times T$-dimensional problem.

Due to the independence of the $\mu_{i}$ 's $\log d e n s i t y \log g(y ; \hat{\xi})$ is given by

$$
\log g(y ; \hat{\xi})=\text { constant }-\frac{1}{2} \sum_{i=1}^{N} \log \left|\operatorname{Var}_{g}\left(\bar{y}_{i} ; \hat{\xi}\right)\right|+\left[\left(\bar{y}_{i}-\bar{c}_{i}-\hat{\mathcal{B}}_{i}\right)^{\prime} \operatorname{Var}_{g}\left(\bar{y}_{i} ; \hat{\xi}\right)^{-1}\left(\bar{y}_{i}-\bar{c}_{i}-\hat{\mathcal{B}}_{i}\right)\right]
$$

where determinant $\left|\operatorname{Var}_{g}\left(\bar{y}_{i} ; \hat{\xi}\right)\right|=\left|\bar{A}_{i} \Sigma_{\mu} \bar{A}_{i}^{\prime}+\bar{D}_{i}\right|$ can be hard to evaluate, depending on the structure of $\bar{A}_{i}$. More efficiency can be obtained by using the collapsed vector series $\bar{y}_{i}^{l}$, for 
$i=1, \ldots, N$. Based on model (15) we obtain

$$
\log g(y ; \hat{\xi})=\text { constant }+\log g\left(\bar{y}^{l} ; \hat{\xi}\right)-\frac{1}{2} \sum_{i=1}^{N} \log \left|\bar{D}_{i}\right|+\bar{e}_{i}^{\prime} D_{i}^{-1} \bar{e}_{i},
$$

where $\bar{e}_{i}=\mathcal{M}_{i}\left(\bar{y}_{i}-\bar{c}_{i}-\hat{\mathcal{B}}_{i}\right)$ with $\mathcal{M}_{i}=I-\bar{A}_{i}\left(\bar{A}_{i}^{\prime} \bar{D}_{i}^{-1} \bar{A}_{i}\right)^{-1} \bar{A}_{i}^{\prime} \bar{D}_{i}^{-1}$. Logdensity $\log g(y ; \hat{\xi})$ can therefore be cased on the $N \times q$-dimensional model (15).

The following algorithm summarizes the evaluation of the loglikelihood for balanced panels. Given parameter vector $\psi$ we can evaluate the Monte Carlo loglikelihood estimate $\log \hat{p}(y)$ in the following steps:

\section{Algorithm B}

(i) Run Algorithm A, where the posterior modal values $\hat{\mu}$ and $\hat{\xi}$ are calculated;

(ii) Collapse panel $y$ into low-dimensional vector series $\bar{y}_{i}^{l}$ and $y_{t}^{l}$;

(iii) Sample $M$ draws $\mu^{(i)}$ and $\xi^{(i)}$ from densities $g\left(\xi \mid y^{l} ; \hat{\mu}\right)$ and $g\left(\mu \mid \bar{y}^{l} ; \hat{\xi}\right)$, which are based on transformed models (11) and (15), and compute importance weights $w^{(i)}$, as given in equation (2);

(iv) Evaluate $\log d e n s i t i e s \log g(y ; \hat{\mu})$ as in (16) and $\log g(y ; \hat{\xi})$ as in (17);

(v) Compute $\log \hat{p}(y)=\log g(y ; \hat{\mu})+\log g(y ; \hat{\xi})+\log M^{-1} \sum_{i=1}^{M} w^{(i)}$.

Loglikelihood estimate $\log \hat{p}(y)$ can be optimized with respect to parameter vector $\psi$ using an arbitrary numerical optimization method. As a practical choice we use the BFGS algorithm, see Nocedal \& Wright (1999). To retain the smoothness of the likelihood in $\psi$ we use the same random seed and the same value of $M$ for each loglikelihood evaluation. The resulting Monte Carlo parameter estimates are denoted by $\tilde{\psi}$. In Section 2 we show the computational efficiency and accuracy of our methods, by providing average estimation times and summary statistics from repeated parameter estimates, for simulated data from different dynamic panel data models. Durbin \& Koopman (1997) advocate the use of antithetic variables to improve the efficiency of the importance sampling weights. An antithetic variable in our context is constructed for each random draw $\mu^{(i)}$ or $\xi^{(i)}$ from the importance densities such that it is equiprobable with $\mu$ or $\xi$, respectively, and it leads to smaller Monte Carlo variation. For each draw of $\mu^{(i)}$ and $\xi^{(i)}$ we manifacture antithetic variables that balance for location and for scale. see Durbin \& Koopman (2012, Section 11.4.3) for a detailed discussion.

\subsection{Estimation of the posterior random effects}

Given the estimated parameter vector $\tilde{\psi}$ we calculate Monte Carlo estimates of the individualspecific and time-varying effects. A more detailed discussion of this approach is given in 
Durbin \& Koopman (2012, Chapter 11). Let $f(\mu, \xi)$ denote a general function of $\mu$ and $\xi$ that is of interest. It holds that

$$
\mathrm{E}_{p}[f(\mu, \xi) \mid y]=\int_{\xi} \int_{\mu} f(\mu, \xi) p(\mu, \xi \mid y ; x) \mathrm{d} \mu \mathrm{d} \xi
$$

where $\mathrm{E}_{p}[\cdot \mid y]$ refers to the expectation with respect to the density $p(\mu, \xi \mid y ; x)$. For given modal values $\hat{\mu}$ and $\hat{\xi}$, the accompanying importance sampling representation is given by

$$
\mathrm{E}_{p}[f(\mu, \xi) \mid y]=p(y)^{-1} \int_{\xi} \int_{\mu} f(\mu, \xi) \frac{p(y \mid \mu, \xi ; x) p(\mu) p(\xi)}{g(\mu \mid y ; \hat{\xi}) g(\xi \mid y ; \hat{\mu})} g(\mu \mid y ; \hat{\xi}) g(\xi \mid y ; \hat{\mu}) \mathrm{d} \mu \mathrm{d} \xi
$$

When applying Bayes rule twice to the right hand side we obtain

$$
\mathrm{E}_{p}[f(\mu, \xi) \mid y]=\frac{g(y ; \hat{\xi}) g(y ; \hat{\mu})}{p(y)} \int_{\xi} \int_{\mu} f(\mu, \xi) w(y, \mu, \xi ; \hat{\mu}, \hat{\xi}) g(\mu \mid y ; \hat{\xi}) g(\xi \mid y ; \hat{\mu}) \mathrm{d} \mu \mathrm{d} \xi
$$

where

$$
w(y, \mu, \xi ; \hat{\mu}, \hat{\xi})=\frac{p(y \mid \mu, \xi ; x)}{g(y \mid \mu ; \hat{\xi}) g(y \mid \xi ; \hat{\mu})} .
$$

Now, when setting $f(\mu, \xi)=1$ we obtain

$$
1=\frac{g(y ; \hat{\xi}) g(y ; \hat{\mu})}{p(y)} \int_{\xi} \int_{\mu} w(y, \mu, \xi ; \hat{\mu}, \hat{\xi}) g(\mu \mid y ; \hat{\xi}) g(\xi \mid y ; \hat{\mu}) \mathrm{d} \mu \mathrm{d} \xi
$$

And when dividing the two equations above we get

$$
\mathrm{E}_{p}[f(\mu, \xi) \mid y]=\frac{\int_{\xi} \int_{\mu} f(\mu, \xi) w(y, \mu, \xi ; \hat{\mu}, \hat{\xi}) g(\mu \mid y ; \hat{\xi}) g(\xi \mid y ; \hat{\mu}) \mathrm{d} \mu \mathrm{d} \xi}{\int_{\xi} \int_{\mu} w(y, \mu, \xi ; \hat{\mu}, \hat{\xi}) g(\mu \mid y ; \hat{\xi}) g(\xi \mid y ; \hat{\mu}) \mathrm{d} \mu \mathrm{d} \xi}
$$

for which a Monte Carlo estimate $\tilde{f}(\mu, \xi)$ is given by

$$
\tilde{f}(\mu, \xi)=\frac{\sum_{i=1}^{M} f\left(\mu^{(i)}, \xi^{(i)}\right) w^{(i)}}{\sum_{i=1}^{M} w^{(i)}},
$$

where $w^{(i)}$ is defined in equation (2).

\section{Simulation Results}

\subsection{Diagnostic tests importance sampling weights}

In Figures 11-9 we present the importance sampling diagnostics for all density, signal and parameters combinations given in Table 1. They are computed as discussed in Mesters \& Koopman (2014, Section 4.2). 
Observation density

\begin{tabular}{l} 
(A) Binary $\quad \log p\left(y_{i, t} \mid z_{i, t}\right)=y_{i, t} z_{i, t}-\log \left(1+\exp z_{i, t}\right)$ \\
(B) Binomial $\log p\left(y_{i, t} \mid z_{i, t}\right)=y_{i, t} z_{i, t}-n_{i, t}\left(1+\exp z_{i, t}\right)-\log \left(\begin{array}{l}n_{i, t} \\
y_{i, t}\end{array}\right)$ \\
(C) Student's $t \log p\left(y_{i, t} \mid z_{i, t}\right)=\log a(\nu)+\frac{1}{2} \log \lambda-\frac{\nu+1}{2} \log \left(1+\lambda\left(y_{i, t}-z_{i, t}\right)^{2}\right)$ \\
\hline
\end{tabular}

\begin{tabular}{llllllll} 
Signal & Parameters \\
\hline & $\gamma$ & $\beta$ & $\sigma_{\mu}$ & $h$ & $\sigma_{\eta}$ & $\nu$ \\
\hline
\end{tabular}

(1) $\quad z_{i, t}=y_{i, t-1} \gamma+x_{i, t}^{\prime} \beta+\mu_{i}$

$\begin{array}{lllllll}\text { a) } & 0.2 & 1 & 0.5 & - & - & (3,5,10) \\ \text { b) } & 0.2 & 1 & 1 & - & - & (3,5,10) \\ \text { c) } & 0.2 & 1 & 3 & - & - & (3,5,10)\end{array}$

$(2)$

$$
z_{i, t}=y_{i, t-1} \gamma+x_{i, t}^{\prime} \beta+\xi_{t} \quad \text { a) } \quad 0.2 \quad 1 \quad-\quad 0.3 \quad 0.2 \quad(3,5,10)
$$

b) $\quad 0.2 \quad 1 \quad-\quad 0.9 \quad 0.2 \quad(3,5,10)$

$(3)$

$$
\begin{array}{rlllllll}
z_{i, t}=y_{i, t-1} \gamma+x_{i, t}^{\prime} \beta+\mu_{i}+\xi_{t} & \text { a) } & 0.2 & 1 & 0.5 & 0.3 & 0.2 & (3,5,10) \\
& \text { b) } & 0.2 & 1 & 0.5 & 0.9 & 0.2 & (3,5,10) \\
& \text { c) } & 0.2 & 1 & 1 & 0.3 & 0.2 & (3,5,10) \\
& \text { d) } & 0.2 & 1 & 1 & 0.9 & 0.2 & (3,5,10) \\
& \text { e) } & 0.2 & 1 & 3 & 0.3 & 0.2 & (3,5,10) \\
\text { f) } & 0.2 & 1 & 3 & 0.9 & 0.2 & (3,5,10)
\end{array}
$$

Table 1: Monte Carlo design with our signal specifications, parameter values and panel dimensions for simulating the observations. The data generation process is further given by $x_{i, t} \sim \operatorname{NID}(0,1)$, $\mu_{i} \sim \mathrm{NID}\left(0, \sigma_{\mu}^{2}\right), \xi_{t}=\alpha_{t}, \alpha_{t+1}=h \alpha_{t}+\eta_{t}$ and $\eta_{t} \sim \mathrm{NID}\left(0, \sigma_{\eta}^{2}\right)$. The initial time varying effect is taken as $N\left(0, \sigma_{\eta}^{2} /\left(1-h^{2}\right)\right)$. For the Student's $t$ density it holds that $a(\nu)=\Gamma(\nu / 2+1 / 2) / \Gamma(\nu / 2)$ and $\lambda^{-1}=(\nu-2) \sigma^{2}$. 

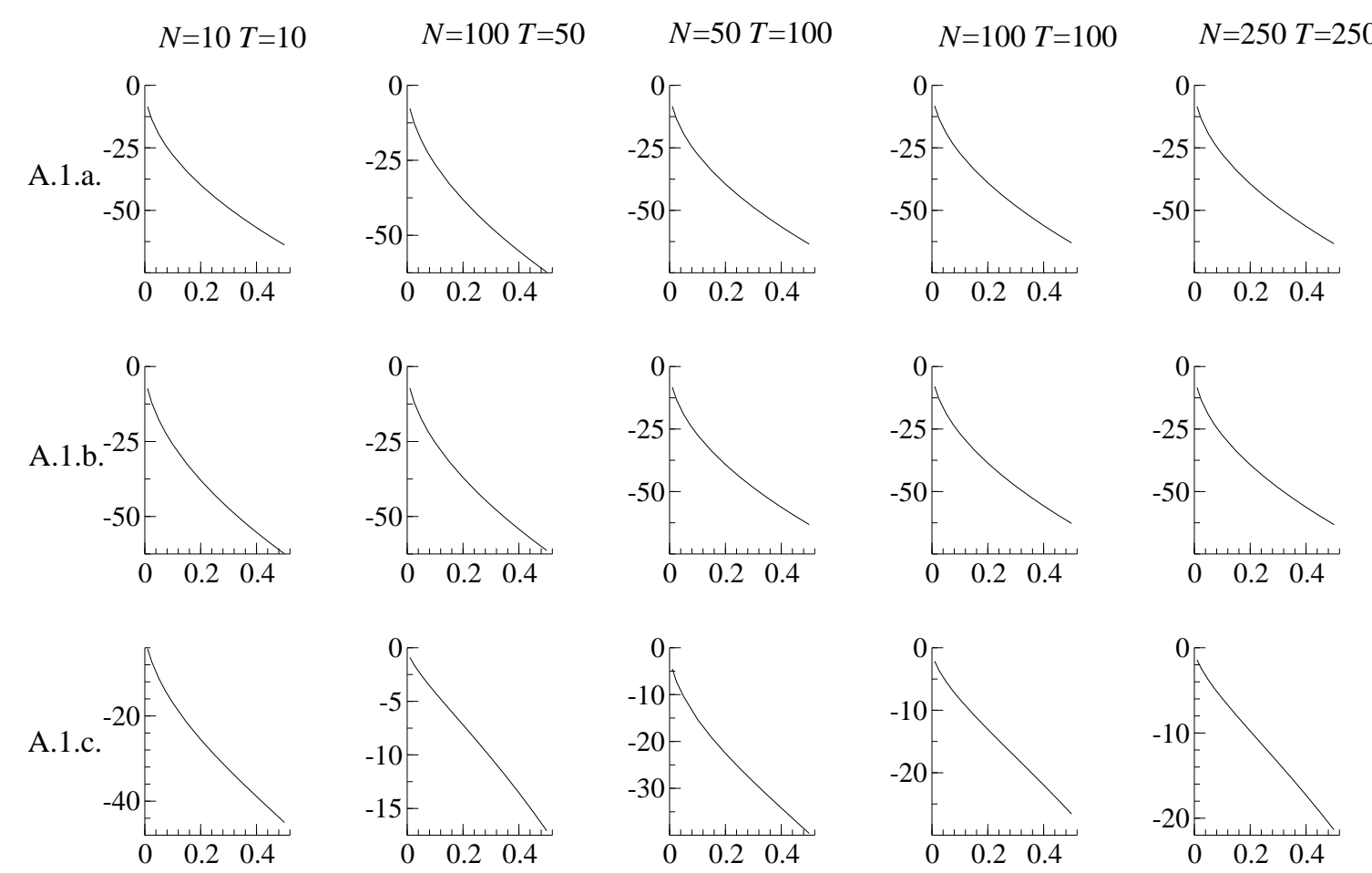

Figure 1: Diagnostic tests Binary signal 1
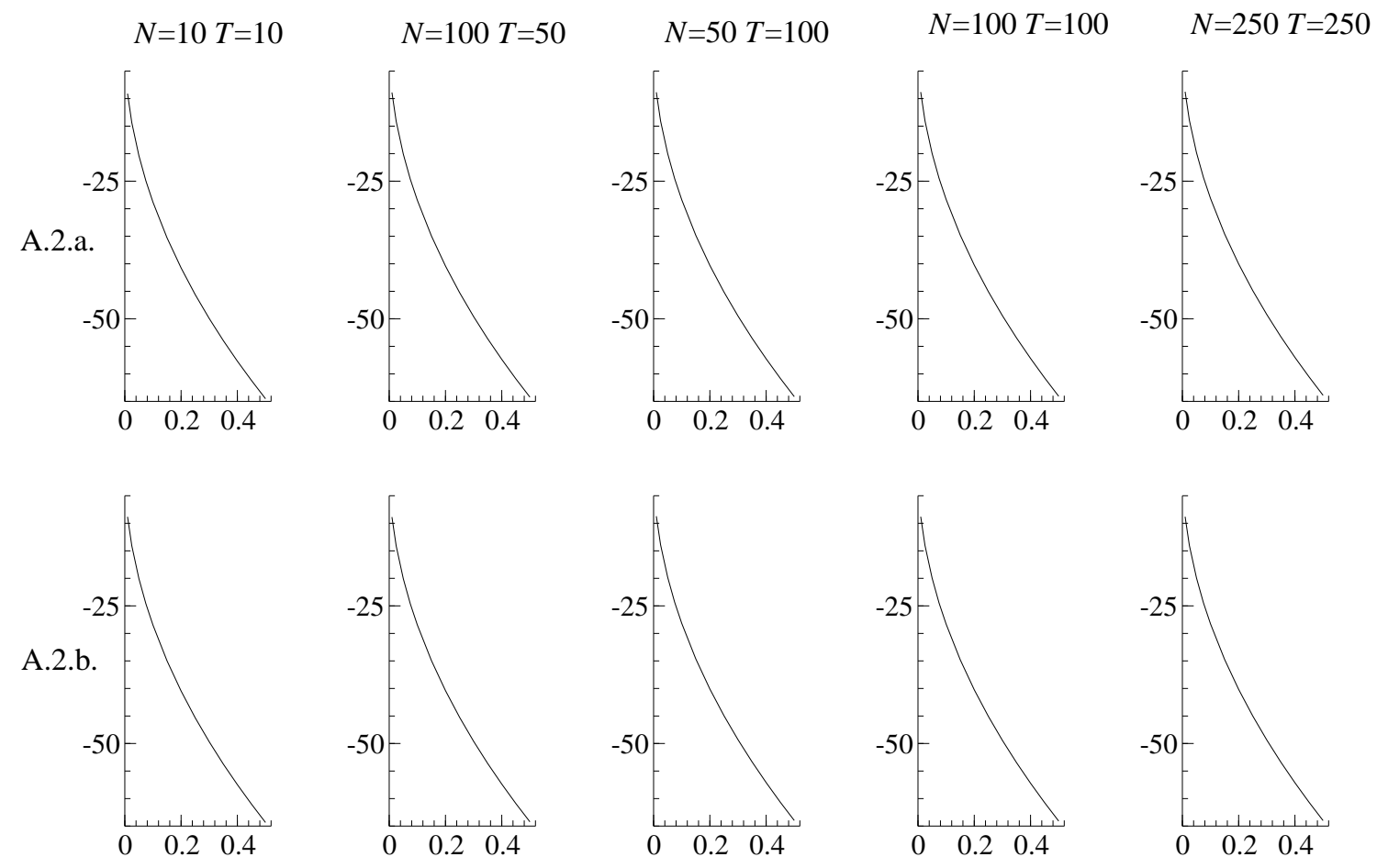

Figure 2: Diagnostic tests Binary signal 2 


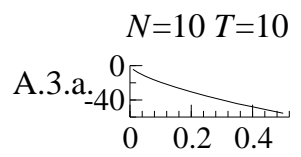
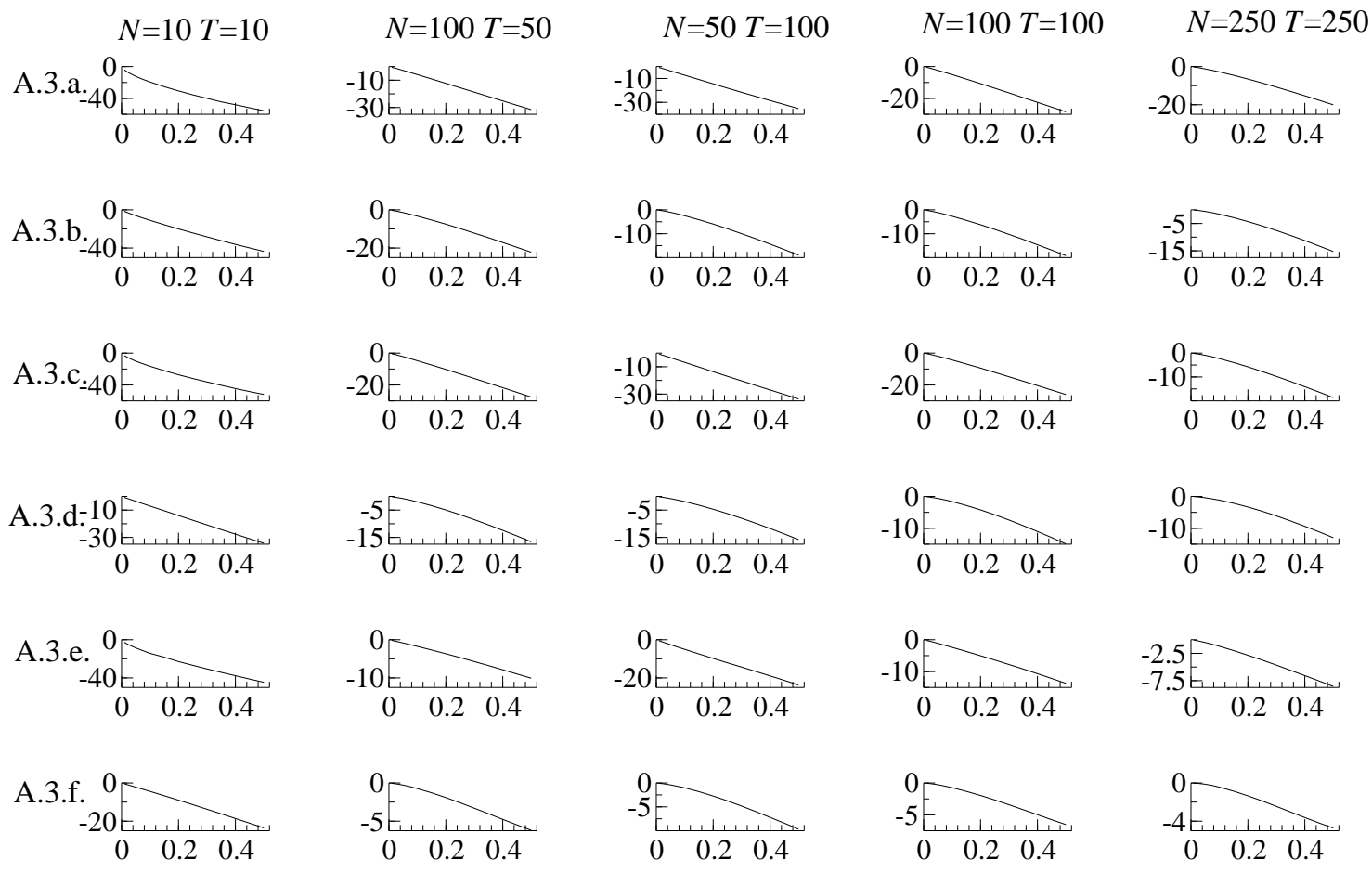

Figure 3: Diagnostic tests Binary signal 3
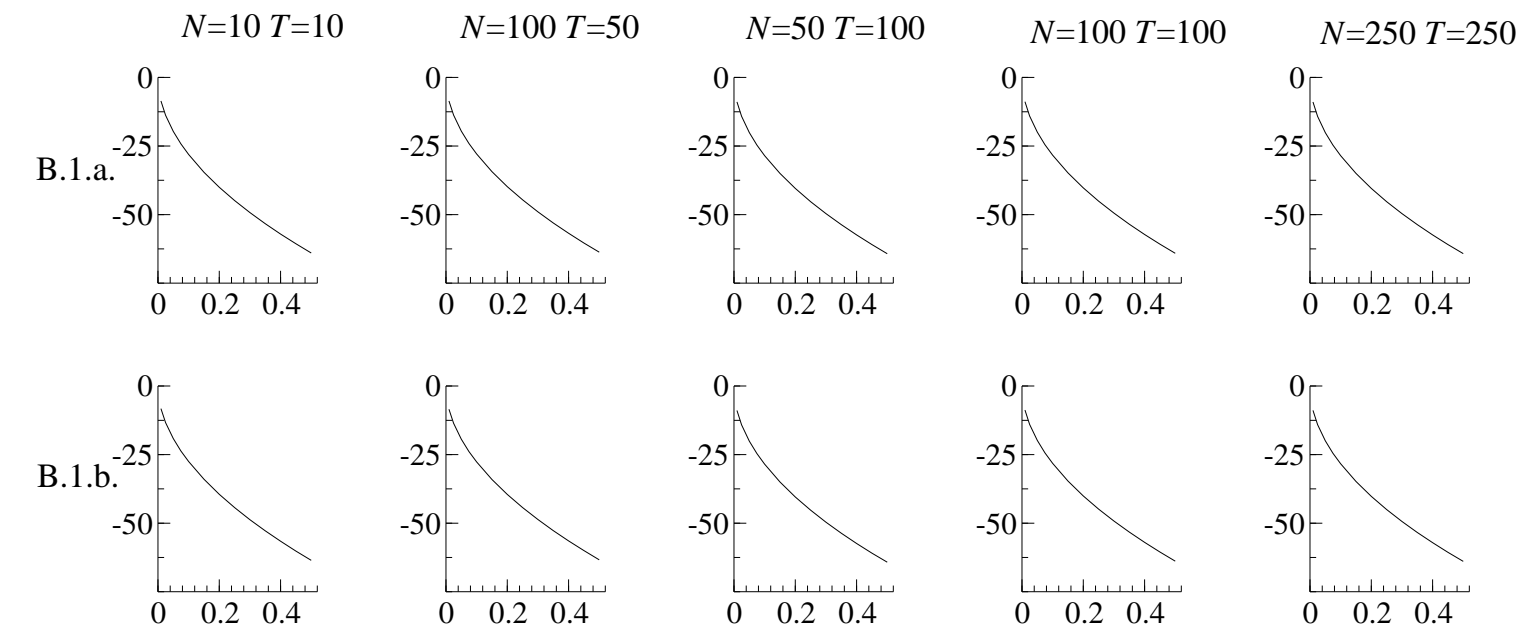

B.1.c.
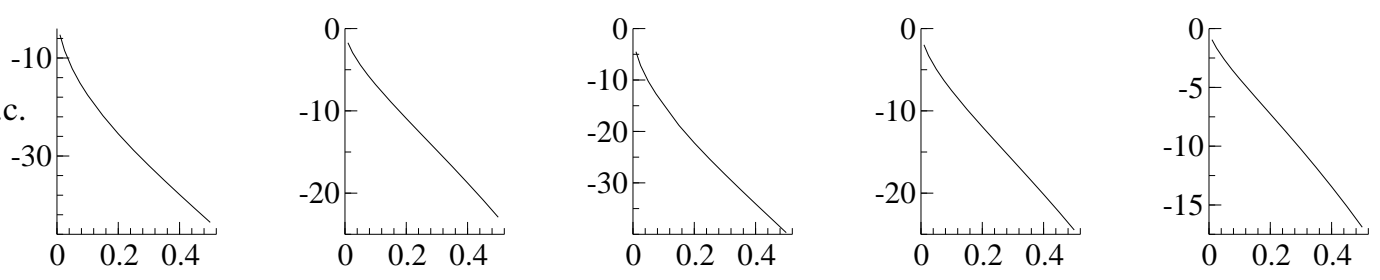

Figure 4: Diagnostic tests Binomial signal 1 

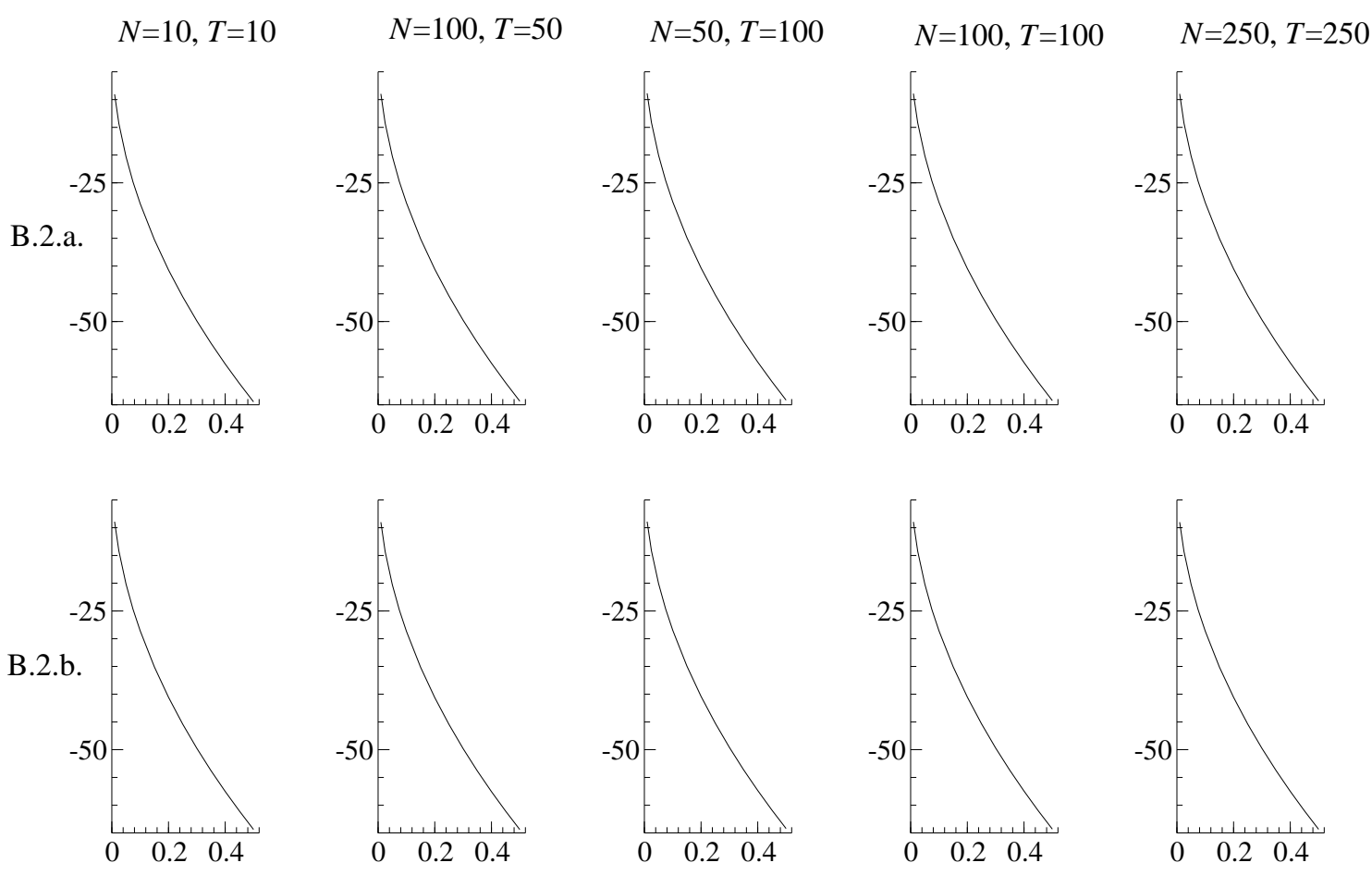

Figure 5: Diagnostic tests Binomial signal 2

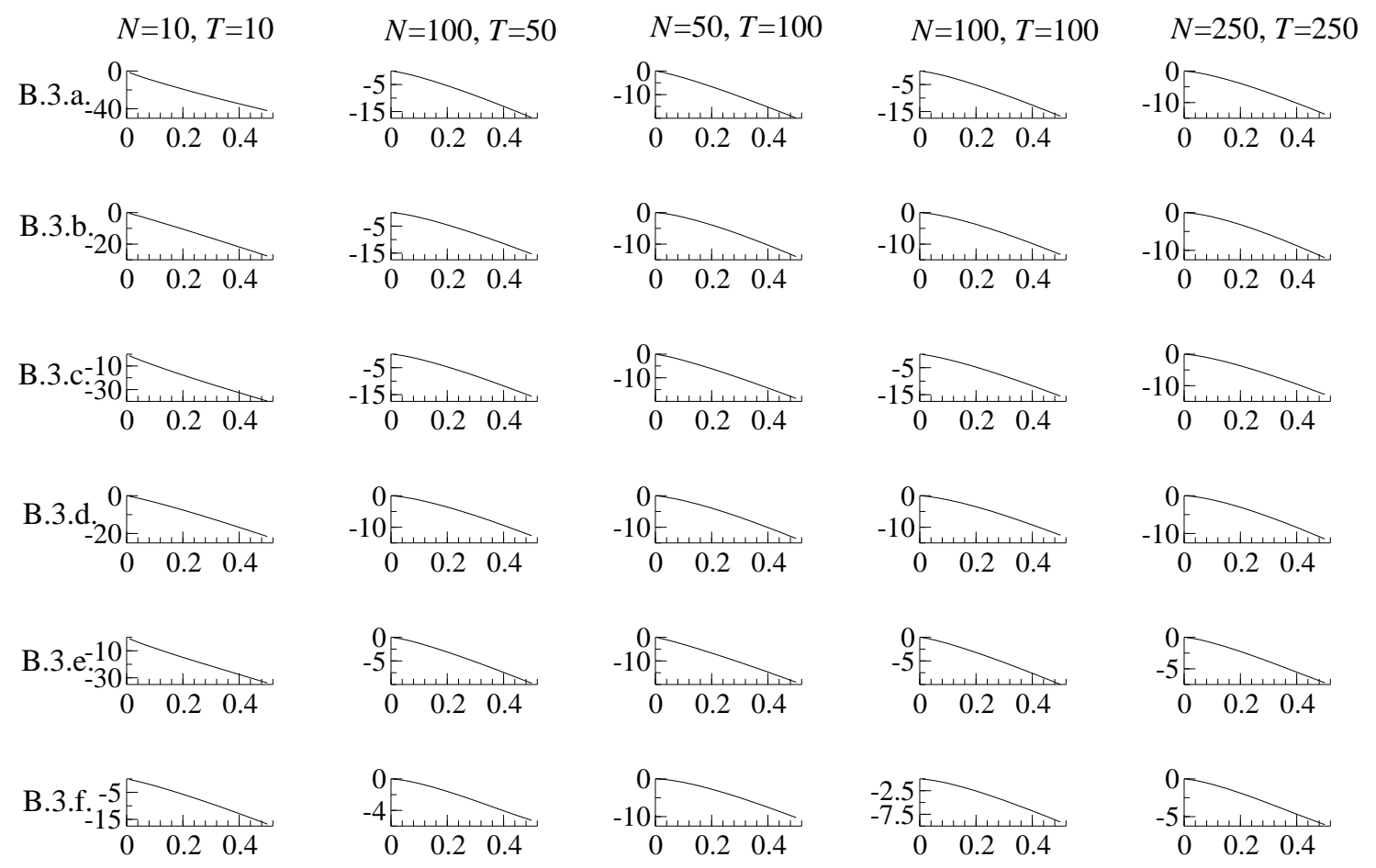

Figure 6: Diagnostic tests Binomial signal 3 

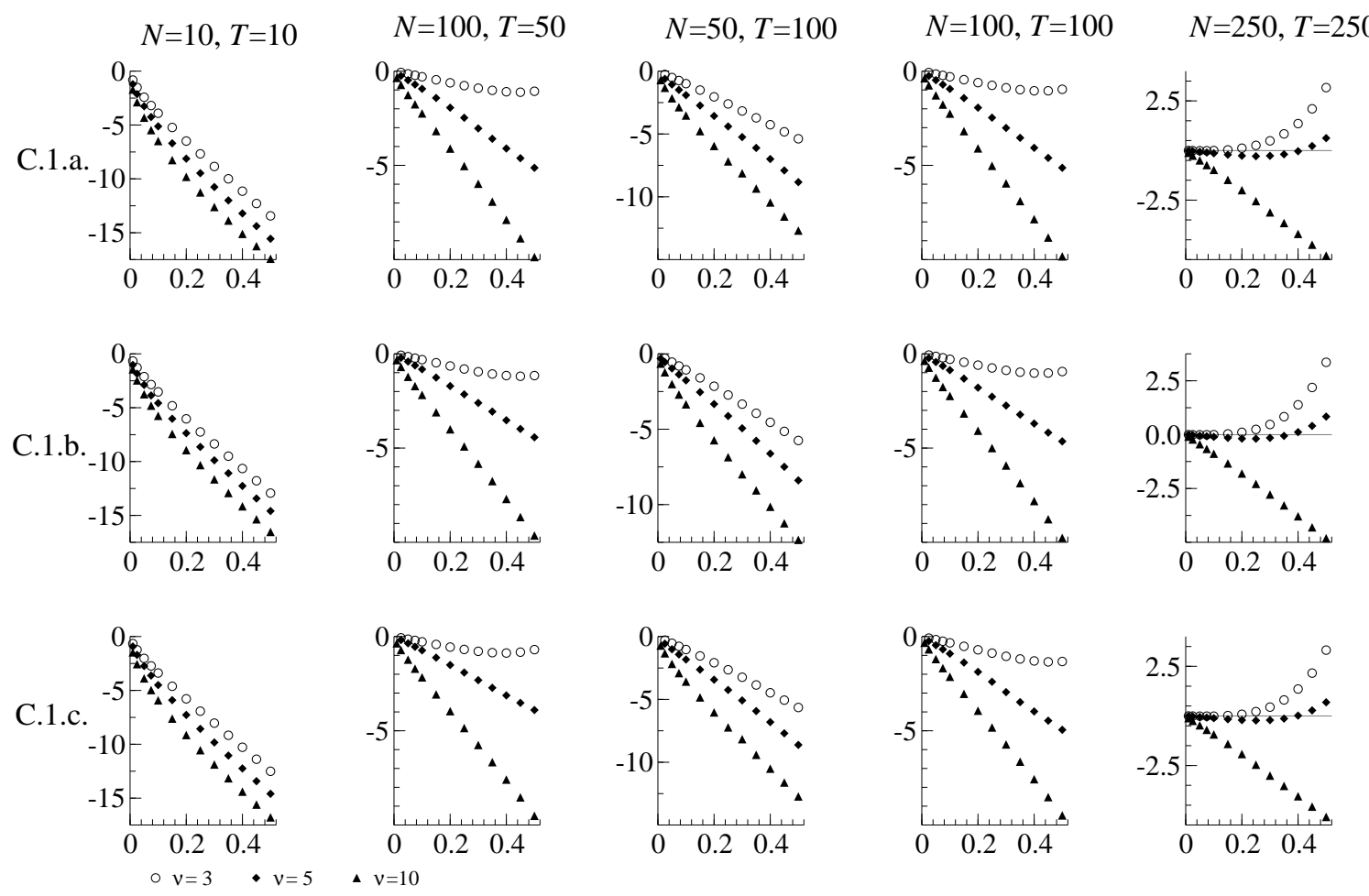

Figure 7: Diagnostic tests Student's $t$ signal 1
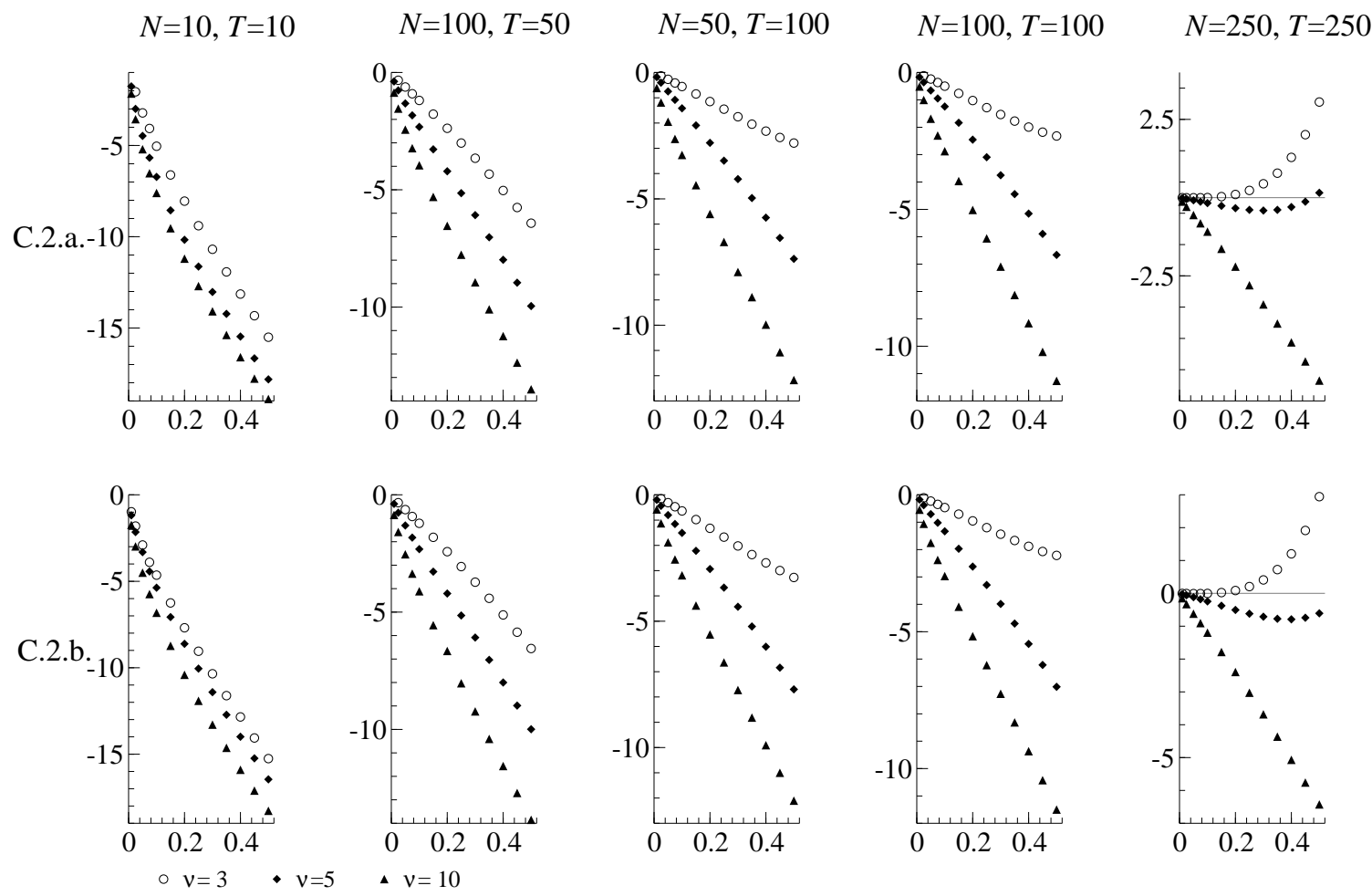

Figure 8: Diagnostic tests Student's $t$ signal 2 


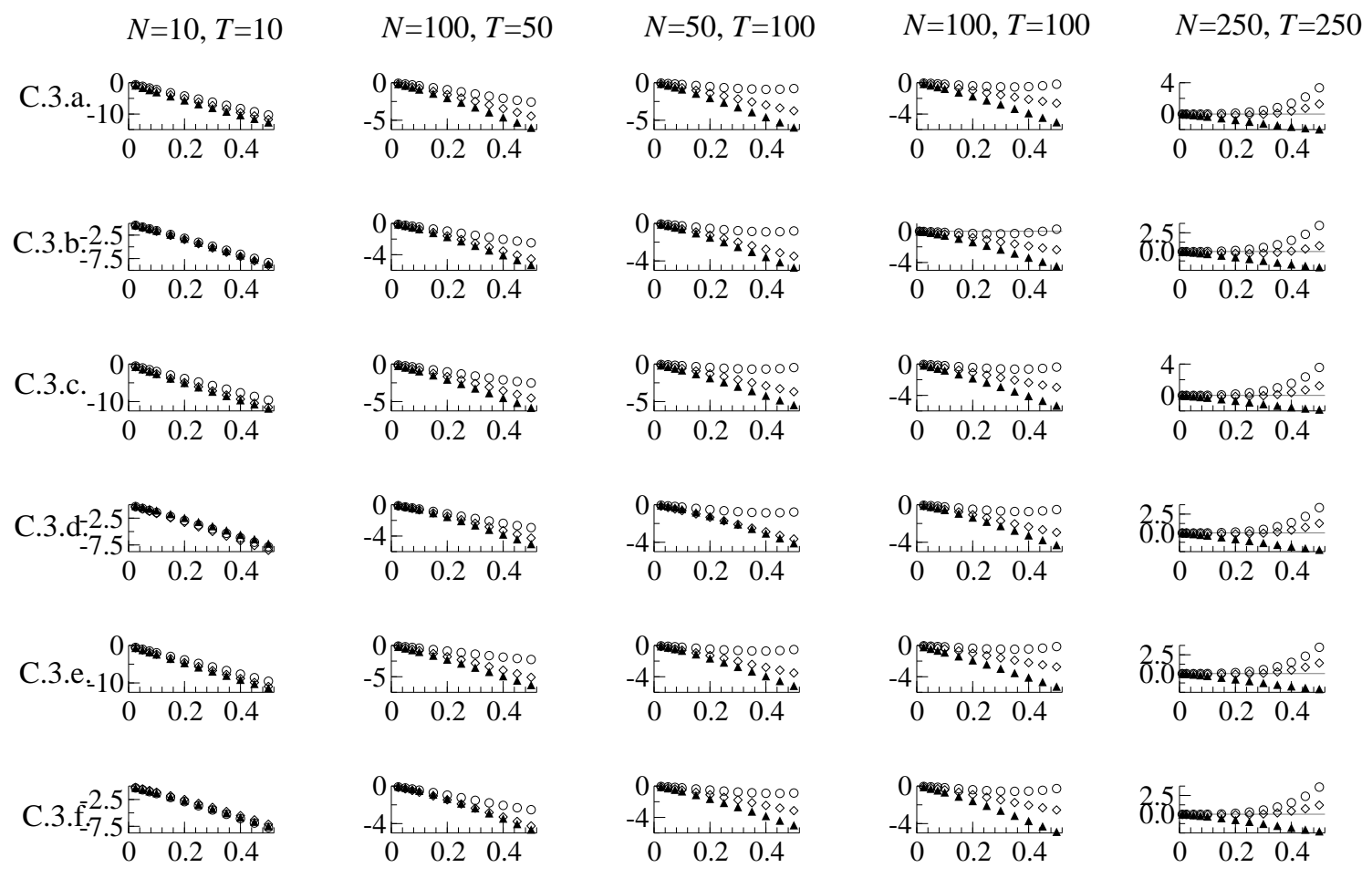

Figure 9: Diagnostic tests Student's $t$ signal 3

\subsection{Efficiency loss}

We discussed in Mesters \& Koopman (2014, Section 3) that our proposed implementation of importance sampling for the generalized dynamic panel data model is different from the standard implementation of Shephard \& Pitt (1997) and Durbin \& Koopman (1997), hereafter SPDK. The standard SPDK method would sample $\mu^{(i)}$ and $\xi^{(i)}$ from $g(\mu, \xi \mid y)$, where $g(\mu, \xi \mid y)$ is based on the linear Gaussian model (3). The corresponding likelihood is then estimated by

$$
\hat{p}(y)=g(y) M^{-1} \sum_{i=1}^{M} w^{(i)}, \quad w^{(i)}=\frac{p\left(y \mid \mu^{(i)}, \xi^{(i)} ; x\right)}{g\left(y \mid \mu^{(i)}, \xi^{(i)}\right)},
$$

where $\mu^{(i)}$ and $\xi^{(i)}$ are drawn from $g(\mu, \xi \mid y)$. This implementation is not feasible for even moderately large panels; see the discussion in Section 1.

It is anticipated that the variance of the importance weight function increases when the SPDK method in (18) is replaced by our method in (1) because Algorithm A does not account for the dependence between $\mu$ and $\xi$ in $g(\mu, \xi \mid y)$. The efficiency loss due to Algorithm A can also be investigated for the small panel. It may provide some insight into a possible necessary increase in the number of draws $M$ compared to SPDK. For this purpose we carry out the the following simulation experiment. We generate fifty data panels based on signal 3 and for each combination of observation density and parameter values reported in Table 1. 
For each of these simulated data panels, we obtain likelihood estimates at the true parameter values under a hundred different random seeds. We are interested in the standard deviation of the likelihood estimates for different values of $M$ and computed by

$$
S D_{M}=(1 / 50) \sum_{i=1}^{50}\left[(1 / 100) \sum_{j=1}^{100}\left(\log \hat{p}_{j}\left(y^{i}\right)-\log \bar{p}\left(y^{i}\right)\right)^{2}\right]^{0.5},
$$

where $\log \bar{p}\left(y^{i}\right)=(1 / 100) \sum_{j=1}^{100} \log \hat{p}_{j}\left(y^{i}\right)$ with $\hat{p}_{j}\left(y^{i}\right)$ as the likelihood estimate based on $M$ draws, for the $j$ th random seed and for the $i$ th panel $y^{i}$.

The resulting values for $S D_{M}$ are reported in Table 2 for $M=50,100,1000$. For the Binary and Binomial densities, we observe a substantial increase in the variance of the likelihood estimate from Algorithm A when compared to the SPDK implementation, for each value of $M$. The increase decreases rapidly when the variance of the individual-specific effect $\sigma_{\mu}^{2}$ increases. For the Student's $t$ densities the increase in the variance is smaller and sometimes, for $\nu=3$, Algorithm $\mathrm{A}$ is more efficient than SPDK. These results are only indicative since we can carry out these comparisons only for small panel dimensions. However, the increase of $S D_{M}$ can be offset by increasing the number of draws $M$. Any degree of accuracy can be achieved and given the large computational improvements, as documented below, this seems a minor difficulty. We notice that SPDK is clearly not feasible for even modest panel dimensions.

\subsection{Likelihood evaluation times}

A major improvement for our simulation based estimator is proposed in Mesters \& Koopman (2014, Section 3.2) where it is shown how large panel and time series data vectors can be collapsed to much smaller dimensions. We investigate the gains in computing time by simulating 100 data panels for each possible design given in Table1. For each simulated data panel, we evaluate the likelihood by Algorithm B as described in Section 1 with $M=1000$. We consider the standard implementation based on vector series $y_{t}$ and $\bar{y}_{i}$ as well as the collapsed implementation based on $y_{t}^{l}$ and $\bar{y}_{i}^{l}$ of Section 1. The average evaluation time for the likelihood for the collapsed implementation and the ratio between the evaluation times for the collapsed and standard implementations is presented in Table 3 for signal 1.b, 2.b and 3.d. The reduction in evaluation times are of course the same for different parameter values. The likelihood evaluation procedure based on the collapsed vectors is between 2 and 10 times faster compared to evaluation without collapsing the vectors. Most computational gains are due to the collapse of panel dimension $N$ for the sampling of time-varying effect $\xi$.

\subsection{Parameter estimation results}

We report the average bias and standard deviation of the parameter estimation results in Tables 4-8 for all densities and signals. See Mesters \& Koopman (2014, Section 4.4) for further details. 


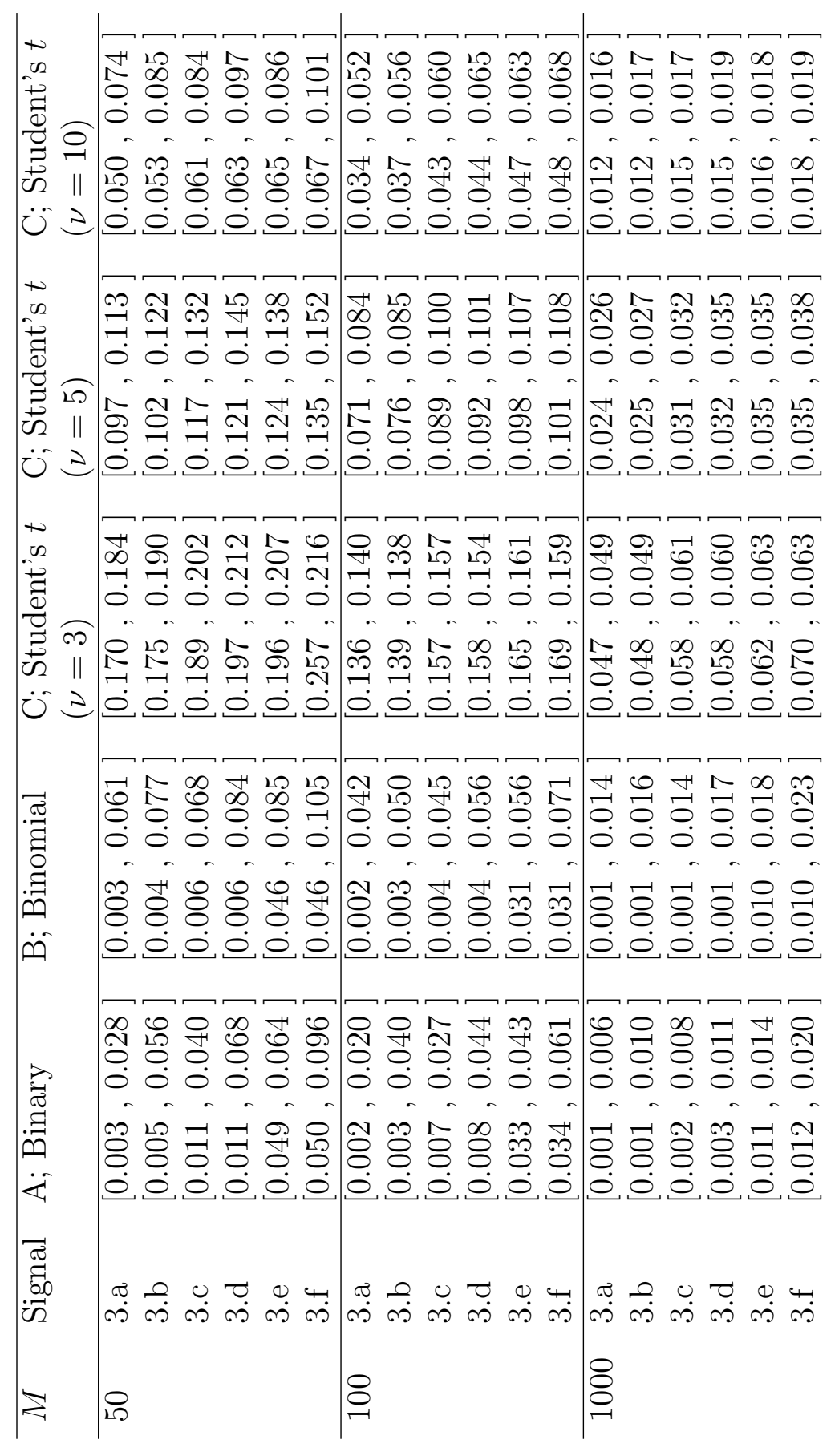

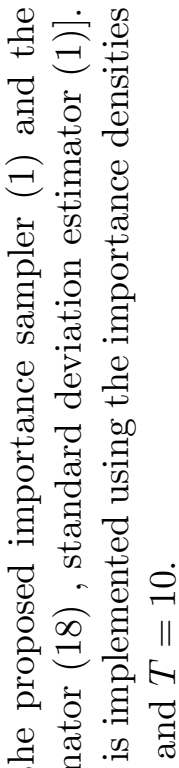

후임잉

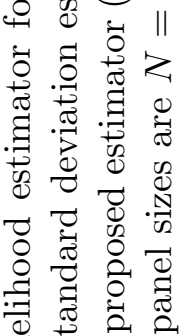

记

을

نี

苛

品范

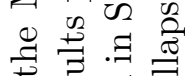

+ क

व \&

兽苛

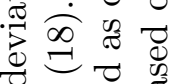

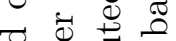

宅 茵

สี

क \&

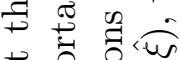

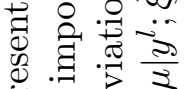

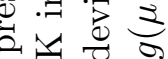

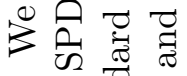

굴

需要

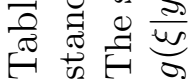




\begin{tabular}{lllll}
\multicolumn{1}{l}{$N=100$} & $N=50$ & $N=100$ & $N=250$ \\
& $T=50$ & $T=100$ & $T=100$ & $T=250$ \\
\hline \multicolumn{5}{c}{ A. Binary } \\
1.b & {$[0.286,0.635]$} & {$[0.281,0.532]$} & {$[0.584,0.568]$} & {$[3.387,0.594]$} \\
2.b & {$[0.334,0.169]$} & {$[0.369,0.164]$} & {$[0.665,0.161]$} & {$[3.705,0.160]$} \\
$3 . d$ & {$[0.591,0.172]$} & {$[0.607,0.178]$} & {$[1.240,0.210]$} & {$[9.688,0.223]$} \\
\hline \multicolumn{5}{c}{ B. Binomial } \\
1.b & {$[0.184,0.733]$} & {$[0.178,0.675]$} & {$[0.361,0.702]$} & {$[2.165,0.692]$} \\
2.b & {$[0.207,0.143]$} & {$[0.226,0.146]$} & {$[0.409,0.145]$} & {$[2.359,0.155]$} \\
$3 . d$ & {$[0.386,0.180]$} & {$[0.401,0.187]$} & {$[0.852,0.187]$} & {$[7.006,0.308]$} \\
\hline \multicolumn{5}{c}{ C. Student's } \\
1.b & {$[0.115,0.657]$} & {$[0.111,0.590]$} & {$[0.228,0.621]$} & {$[1.339,0.597]$} \\
2.b & {$[0.191,0.100]$} & {$[0.207,0.102]$} & {$[0.379,0.102]$} & {$[2.129,0.098]$} \\
3.d & {$[0.663,0.164]$} & {$[0.797,0.222]$} & {$[1.692,0.221]$} & {$[11.504,0.280]$} \\
\hline
\end{tabular}

Table 3: Average likelihood evaluation time for the collapsed likelihood implementation (left in seconds) and the average ratio between the the collapsed and standard implementations of the likelihood evaluation as discussed above (the average is over 100 panels). The reduction is achieved by sampling from $\mu^{(i)}$ and $\xi^{(i)}$ from $g\left(\xi \mid y^{l} ; \hat{\mu}\right)$ and $g\left(\mu \mid y^{l} ; \hat{\xi}\right)$ instead of from $g(\xi \mid y ; \hat{\mu})$ and $g(\mu \mid y ; \hat{\xi})$, respectively. The signals are taken as in Table 1. For each model the likelihood is evaluated as discussed in Section 1 and by using $M=1000$ samples from the importance densities. For the Student's $t$ density the 5 degrees of freedom were used.

\section{$3 \quad$ Weights empirical studies}

In Figure 10 we present the diagnostic tests for the weights pertaining to the empirical studies. The weights are computed as discussed in Mesters \& Koopman (2014, Section 4.2). 


\begin{tabular}{|c|c|c|c|c|c|c|c|}
\hline Code & $N$ & $T$ & $\gamma$ & $\beta$ & $\sigma_{\mu}$ & $h$ & $\sigma_{\eta}$ \\
\hline \multirow[t]{4}{*}{ A.1.a. } & 100 & 50 & -0.0030 .059 & 0.0030 .039 & -0.0060 .059 & & \\
\hline & 50 & 100 & -0.0070 .059 & $-0.001 \quad 0.038$ & -0.0020 .056 & & \\
\hline & 100 & 100 & -0.0080 .047 & $\begin{array}{ll}-0.001 & 0.026\end{array}$ & 0.0020 .041 & & \\
\hline & 250 & 250 & $0.002 \quad 0.017$ & -0.0010 .012 & $0.004 \quad 0.024$ & & \\
\hline \multirow[t]{4}{*}{ A.1.b. } & 100 & 50 & 0.0010 .076 & $0.001 \quad 0.038$ & -0.0010 .084 & & \\
\hline & 50 & 100 & -0.0090 .062 & -0.0010 .038 & -0.0010 .101 & & \\
\hline & 100 & 100 & -0.0080 .051 & $\begin{array}{lll}-0.001 & 0.027\end{array}$ & $0.004 \quad 0.074$ & & \\
\hline & 250 & 250 & $0.000 \quad 0.018$ & -0.0020 .011 & $0.008 \quad 0.044$ & & \\
\hline \multirow[t]{4}{*}{ A.1.c. } & 100 & 50 & 0.0010 .113 & 0.0020 .045 & -0.0190 .230 & & \\
\hline & 50 & 100 & -0.0030 .094 & -0.0050 .049 & -0.0080 .352 & & \\
\hline & 100 & 100 & -0.0030 .067 & $-0.000 \quad 0.038$ & 0.0220 .203 & & \\
\hline & 250 & 250 & 0.0030 .026 & $0.000 \quad 0.013$ & 0.0150 .133 & & \\
\hline \multirow[t]{4}{*}{ A.2.a. } & 100 & 50 & -0.0060 .052 & $\begin{array}{ll}-0.000 & 0.038\end{array}$ & & -0.0390 .333 & -0.0210 .053 \\
\hline & 50 & 100 & 0.0030 .045 & 0.0040 .036 & & -0.1060 .353 & -0.0090 .053 \\
\hline & 100 & 100 & -0.0030 .042 & $0.000 \quad 0.025$ & & -0.0200 .188 & -0.0050 .035 \\
\hline & 250 & 250 & 0.0010 .014 & -0.0020 .011 & & -0.0040 .097 & 0.0000 .013 \\
\hline \multirow[t]{4}{*}{ A.2.b. } & 100 & 50 & 0.0020 .057 & -0.0020 .036 & & -0.0380 .114 & -0.0090 .059 \\
\hline & 50 & 100 & -0.0040 .062 & 0.0030 .033 & & -0.0210 .070 & 0.0010 .041 \\
\hline & 100 & 100 & -0.0050 .051 & $-0.000 \quad 0.026$ & & -0.0250 .070 & $0.004 \quad 0.034$ \\
\hline & 250 & 250 & 0.0010 .018 & -0.0020 .011 & & -0.0080 .028 & 0.0010 .013 \\
\hline \multirow[t]{4}{*}{ A.3.a. } & 100 & 50 & -0.0030 .061 & 0.0010 .039 & 0.0030 .055 & -0.0260 .290 & -0.0120 .053 \\
\hline & 50 & 100 & -0.0080 .063 & -0.0020 .039 & -0.0090 .056 & $-0.007 \quad 0.263$ & -0.0090 .055 \\
\hline & 100 & 100 & $0.006 \quad 0.041$ & 0.0050 .031 & -0.0060 .044 & -0.0490 .227 & 0.0020 .038 \\
\hline & 250 & 250 & $0.000 \quad 0.020$ & -0.0000 .010 & -0.0030 .024 & -0.0050 .090 & -0.0020 .015 \\
\hline \multirow[t]{4}{*}{ A.3.b. } & 100 & 50 & -0.0040 .072 & 0.0010 .036 & -0.0020 .057 & -0.0570 .127 & 0.0010 .042 \\
\hline & 50 & 100 & -0.0060 .065 & -0.0040 .041 & -0.0120 .058 & -0.0320 .085 & -0.0010 .038 \\
\hline & 100 & 100 & 0.0040 .045 & 0.0040 .032 & -0.0040 .043 & -0.0340 .081 & 0.0020 .037 \\
\hline & 250 & 250 & -0.0010 .019 & -0.0000 .010 & -0.0040 .024 & -0.0070 .035 & -0.0010 .016 \\
\hline \multirow[t]{4}{*}{ A.3.c. } & 100 & 50 & -0.0020 .070 & 0.0030 .039 & -0.0030 .091 & -0.0310 .311 & -0.0140 .050 \\
\hline & 50 & 100 & -0.0130 .074 & -0.0060 .043 & -0.0160 .100 & -0.0020 .280 & -0.0120 .058 \\
\hline & 100 & 100 & 0.0070 .045 & 0.0040 .032 & -0.0070 .073 & -0.0320 .234 & -0.0010 .042 \\
\hline & 250 & 250 & -0.0010 .021 & -0.0000 .011 & -0.0050 .047 & -0.0100 .093 & -0.0010 .015 \\
\hline \multirow[t]{4}{*}{ A.3.d. } & 100 & 50 & -0.0030 .076 & 0.0020 .037 & -0.0040 .094 & -0.0640 .131 & 0.0020 .046 \\
\hline & 50 & 100 & -0.0080 .078 & -0.0050 .045 & -0.0220 .104 & -0.0440 .095 & 0.0010 .043 \\
\hline & 100 & 100 & 0.0050 .048 & 0.0040 .032 & $-0.010 \quad 0.074$ & -0.0360 .080 & -0.0010 .038 \\
\hline & 250 & 250 & -0.0000 .020 & -0.0010 .010 & -0.0060 .043 & -0.0090 .037 & -0.0010 .015 \\
\hline \multirow[t]{4}{*}{ A.3.e. } & 100 & 50 & 0.0040 .100 & 0.0040 .046 & -0.0280 .271 & -0.0270 .379 & -0.0330 .096 \\
\hline & 50 & 100 & -0.0190 .100 & -0.0010 .052 & -0.0550 .303 & -0.0590 .357 & -0.0150 .073 \\
\hline & 100 & 100 & -0.0030 .061 & 0.0030 .035 & $\begin{array}{lll}-0.021 & 0.234\end{array}$ & -0.0400 .325 & -0.0190 .067 \\
\hline & 250 & 250 & 0.0020 .029 & 0.0010 .013 & -0.0210 .138 & $-0.019_{0.112}$ & -0.0000 .018 \\
\hline \multirow[t]{4}{*}{ A.3.f. } & 100 & 50 & -0.0010 .102 & $0.008 \quad 0.049$ & -0.0180 .273 & -0.1260 .216 & 0.0090 .069 \\
\hline & 50 & 100 & -0.0080 .104 & -0.0050 .050 & -0.0550 .301 & -0.0910 .144 & 0.0050 .083 \\
\hline & 100 & 100 & $-0.000 \quad 0.064$ & 0.0020 .039 & -0.0320 .245 & -0.0350 .089 & -0.0050 .054 \\
\hline & 250 & 250 & 0.0010 .030 & -0.0000 .012 & $\begin{array}{lll}-0.026 & 0.141\end{array}$ & -0.0140 .041 & -0.0000 .018 \\
\hline
\end{tabular}

Table 4: Simulation results for Binary dynamic panel data models. 


\begin{tabular}{|c|c|c|c|c|c|c|c|}
\hline & $N$ & $T$ & $\gamma$ & $\beta$ & $\sigma_{\mu}$ & $h$ & $\sigma_{\eta}$ \\
\hline \multirow[t]{4}{*}{ B.1.a. } & 100 & 50 & 0.0000 .010 & 0.0010 .018 & -0.0010 .041 & & \\
\hline & 50 & 100 & $-0.000 \quad 0.012$ & -0.0010 .017 & -0.0070 .050 & & \\
\hline & 100 & 100 & $0.000 \quad 0.007$ & 0.0020 .014 & 0.0020 .043 & & \\
\hline & 250 & 250 & -0.0000 .003 & -0.0010 .005 & 0.0000 .020 & & \\
\hline \multirow[t]{4}{*}{ B.1.b. } & 100 & 50 & 0.0010 .012 & 0.0030 .018 & $0.000 \quad 0.077$ & & \\
\hline & 50 & 100 & -0.0010 .014 & -0.0010 .018 & -0.0140 .097 & & \\
\hline & 100 & 100 & 0.0000 .008 & 0.0030 .013 & 0.0010 .082 & & \\
\hline & 250 & 250 & $-0.000 \quad 0.004$ & -0.0000 .006 & $0.000 \quad 0.039$ & & \\
\hline \multirow[t]{4}{*}{ B.1.c. } & 100 & 50 & 0.0020 .019 & 0.0030 .026 & -0.0010 .231 & & \\
\hline & 50 & 100 & -0.0000 .016 & -0.0020 .023 & -0.0420 .309 & & \\
\hline & 100 & 100 & -0.0000 .011 & 0.0010 .017 & $0.010 \quad 0.259$ & & \\
\hline & 250 & 250 & -0.0010 .005 & -0.0000 .008 & 0.0010 .122 & & \\
\hline \multirow[t]{4}{*}{ B.2.a. } & 100 & 50 & -0.0010 .010 & 0.0000 .017 & & -0.0450 .168 & -0.0060 .027 \\
\hline & 50 & 100 & -0.0010 .009 & 0.0010 .018 & & -0.0360 .146 & -0.0020 .025 \\
\hline & 100 & 100 & 0.0010 .006 & 0.0010 .015 & & -0.0320 .123 & $\begin{array}{ll}-0.000 & 0.021\end{array}$ \\
\hline & 250 & 250 & -0.0000 .003 & -0.0010 .005 & & 0.0010 .061 & $\begin{array}{ll}-0.000 & 0.009\end{array}$ \\
\hline \multirow[t]{4}{*}{ B.2.b. } & 100 & 50 & -0.0010 .012 & -0.0010 .018 & & -0.0320 .079 & $-0.003 \quad 0.029$ \\
\hline & 50 & 100 & -0.0020 .010 & 0.0020 .018 & & -0.0230 .068 & -0.0020 .024 \\
\hline & 100 & 100 & 0.0010 .008 & 0.0020 .014 & & -0.0250 .053 & 0.0010 .022 \\
\hline & 250 & 250 & $\begin{array}{lll}-0.000 & 0.003\end{array}$ & -0.0010 .005 & & -0.0070 .026 & 0.0010 .010 \\
\hline \multirow[t]{4}{*}{ B.3.a. } & 100 & 50 & 0.0020 .012 & 0.0010 .020 & -0.0080 .040 & -0.0250 .178 & $\begin{array}{ll}-0.005 & 0.027\end{array}$ \\
\hline & 50 & 100 & 0.0020 .011 & 0.0020 .023 & -0.0050 .047 & -0.0310 .164 & $\begin{array}{ll}-0.000 & 0.025\end{array}$ \\
\hline & 100 & 100 & 0.0000 .008 & 0.0010 .014 & 0.0020 .038 & -0.0340 .129 & -0.0000 .020 \\
\hline & 250 & 250 & 0.0000 .003 & 0.0010 .006 & -0.0000 .024 & 0.0020 .065 & -0.0010 .009 \\
\hline \multirow[t]{4}{*}{ B.3.b. } & 100 & 50 & 0.0030 .012 & $\begin{array}{ll}-0.001 & 0.023\end{array}$ & $\begin{array}{ll}-0.011 & 0.037\end{array}$ & -0.0420 .092 & $\begin{array}{ll}-0.000 & 0.030\end{array}$ \\
\hline & 50 & 100 & 0.0020 .013 & 0.0020 .021 & -0.0090 .047 & -0.0190 .056 & 0.0020 .027 \\
\hline & 100 & 100 & $-0.000 \quad 0.009$ & 0.0020 .014 & $-0.000 \quad 0.037$ & -0.0260 .064 & -0.0010 .021 \\
\hline & 250 & 250 & 0.0000 .003 & $0.001_{0.005}$ & -0.0010 .024 & -0.0070 .031 & -0.0010 .010 \\
\hline \multirow[t]{4}{*}{ B.3.c. } & 100 & 50 & 0.0020 .014 & $0.000 \quad 0.022$ & -0.0110 .073 & -0.0290 .179 & $\begin{array}{ll}-0.004 & 0.029\end{array}$ \\
\hline & 50 & 100 & 0.0030 .014 & 0.0010 .022 & -0.0100 .098 & -0.0450 .167 & $\begin{array}{ll}-0.001 & 0.026\end{array}$ \\
\hline & 100 & 100 & 0.0010 .009 & 0.0000 .015 & 0.0030 .076 & -0.0380 .131 & $\begin{array}{ll}-0.003 & 0.021\end{array}$ \\
\hline & 250 & 250 & 0.0000 .003 & 0.0000 .006 & -0.0020 .048 & 0.0030 .066 & -0.0010 .010 \\
\hline \multirow[t]{4}{*}{ B.3.d. } & 100 & 50 & 0.0030 .015 & 0.0010 .023 & -0.0140 .072 & -0.0480 .101 & $\begin{array}{ll}-0.001 & 0.031\end{array}$ \\
\hline & 50 & 100 & 0.0020 .014 & 0.0010 .020 & -0.0170 .095 & -0.0250 .063 & 0.0010 .028 \\
\hline & 100 & 100 & 0.0000 .009 & 0.0010 .015 & 0.0010 .075 & -0.0280 .064 & $\begin{array}{ll}-0.002 & 0.022\end{array}$ \\
\hline & 250 & 250 & 0.0000 .004 & 0.0000 .006 & -0.0030 .045 & -0.0090 .033 & -0.0010 .010 \\
\hline \multirow[t]{4}{*}{ B.3.e. } & 100 & 50 & 0.0020 .018 & 0.0050 .028 & -0.0130 .224 & -0.0340 .207 & -0.0050 .037 \\
\hline & 50 & 100 & 0.0050 .020 & -0.0010 .027 & -0.0210 .300 & -0.0450 .200 & -0.0050 .037 \\
\hline & 100 & 100 & 0.0020 .013 & 0.0000 .019 & 0.0020 .225 & -0.0350 .160 & -0.0050 .025 \\
\hline & 250 & 250 & $0.000 \quad 0.004$ & $0.000 \quad 0.007$ & -0.0080 .142 & $0.000 \quad 0.073$ & -0.0020 .010 \\
\hline \multirow[t]{4}{*}{ B.3.f. } & 100 & 50 & 0.0030 .017 & 0.0040 .028 & $\begin{array}{ll}-0.021 & 0.225\end{array}$ & -0.0680 .107 & 0.0020 .039 \\
\hline & 50 & 100 & 0.0050 .020 & $0.001 \quad 0.026$ & -0.0310 .304 & -0.0400 .071 & 0.0020 .033 \\
\hline & 100 & 100 & 0.0020 .011 & 0.0000 .020 & $-0.000 \quad 0.215$ & -0.0470 .080 & $\begin{array}{ll}-0.002 & 0.027\end{array}$ \\
\hline & 250 & 250 & 0.0000 .005 & 0.0010 .007 & -0.0070 .142 & -0.0120 .034 & -0.0010 .010 \\
\hline
\end{tabular}

Table 5: Simulation results for Binomial dynamic panel data models. 


\begin{tabular}{|c|c|c|c|c|c|c|c|c|}
\hline & $N$ & $T$ & $\gamma$ & $\beta$ & $\sigma_{\mu}$ & $h$ & $\sigma_{\eta}$ & $\nu$ \\
\hline \multirow[t]{5}{*}{ C.1.a. } & 100 & 50 & -0.0010 .008 & 0.0030 .008 & 0.0120 .035 & & & 0.0280 .034 \\
\hline & 50 & 100 & -0.0010 .006 & -0.0040 .012 & $0.011_{0.054}$ & & & 0.0180 .050 \\
\hline & 100 & 100 & $0.000_{0.007}$ & $0.001_{0.007}$ & -0.0020 .030 & & & $0.014_{0.027}$ \\
\hline & 250 & 250 & 0.0010 .001 & 0.0010 .002 & 0.0040 .016 & & & -0.0020 .008 \\
\hline & 100 & 50 & 0.0000 .008 & 0.0030 .008 & $0.040 \quad 0.076$ & & & $0.028 \quad 0.034$ \\
\hline \multirow[t]{3}{*}{ C.1.b. } & 50 & 100 & -0.0010 .006 & -0.0040 .012 & 0.0240 .104 & & & $0.018 \quad 0.051$ \\
\hline & 100 & 100 & $0.000 \quad 0.007$ & $\begin{array}{lll}0.001 & 0.007\end{array}$ & -0.0020 .054 & & & $0.014 \quad 0.027$ \\
\hline & 250 & 250 & $0.001_{0.001}$ & $0.001_{0.002}$ & 0.0070 .032 & & & -0.0020 .008 \\
\hline \multirow[t]{4}{*}{ C.1.c } & 100 & 50 & $0.000_{0.008}$ & 0.0030 .008 & $0.122_{0.224}$ & & & $0.028_{0.035}$ \\
\hline & 50 & 100 & -0.0010 .006 & -0.0040 .012 & $0.075_{0.305}$ & & & $0.018 \quad 0.051$ \\
\hline & 100 & 100 & -0.0000 .006 & $\begin{array}{lll}0.001 & 0.007\end{array}$ & $-0.000 \quad 0.153$ & & & $0.014_{0.027}$ \\
\hline & 250 & 250 & 0.0010 .001 & 0.0010 .002 & 0.0210 .096 & & & -0.0020 .008 \\
\hline \multirow[t]{4}{*}{ C.2.a. } & 100 & 50 & 0.0010 .005 & -0.0040 .013 & & $0.030_{0.122}$ & 0.0030 .025 & 0.0070 .045 \\
\hline & 50 & 100 & -0.0010 .008 & 0.0040 .007 & & -0.0400 .138 & $0.005 \quad 0.013$ & 0.0250 .033 \\
\hline & 100 & 100 & $-0.000 \quad 0.007$ & -0.0020 .007 & & -0.0300 .093 & -0.0030 .014 & $0.011_{0.032}$ \\
\hline & 250 & 250 & 0.0010 .001 & 0.0010 .002 & & -0.0050 .051 & $0.000 \quad 0.007$ & -0.0040 .007 \\
\hline \multirow[t]{4}{*}{ C.2.b. } & 100 & 50 & 0.0010 .006 & -0.0030 .014 & & -0.0070 .036 & 0.0030 .025 & $0.010 \quad 0.044$ \\
\hline & 50 & 100 & -0.0010 .008 & 0.0040 .007 & & -0.0190 .044 & 0.0050 .014 & $0.024 \quad 0.033$ \\
\hline & 100 & 100 & $-0.000 \quad 0.007$ & -0.0020 .007 & & -0.0210 .043 & -0.0040 .013 & 0.0110 .031 \\
\hline & 250 & 250 & 0.0010 .001 & 0.0010 .002 & & -0.0030 .031 & -0.0000 .006 & -0.0040 .007 \\
\hline \multirow[t]{4}{*}{ C.3.a. } & 100 & 50 & 0.0030 .006 & $-0.000 \quad 0.013$ & -0.0020 .050 & -0.0460 .211 & -0.0060 .029 & 0.0240 .045 \\
\hline & 50 & 100 & 0.0020 .005 & -0.0010 .013 & 0.0030 .075 & -0.0320 .117 & -0.0030 .017 & 0.0210 .043 \\
\hline & 100 & 100 & -0.0010 .006 & 0.0030 .008 & $0.000 \quad 0.030$ & $0.010_{0.067}$ & -0.0090 .017 & $0.018 \quad 0.031$ \\
\hline & 250 & 250 & 0.0000 .001 & 0.0010 .003 & 0.0040 .015 & 0.0140 .062 & -0.0020 .007 & -0.0030 .008 \\
\hline \multirow[t]{4}{*}{ C.3.b. } & 100 & 50 & 0.0030 .006 & -0.0000 .013 & 0.0060 .046 & -0.0880 .155 & -0.0020 .030 & 0.0170 .046 \\
\hline & 50 & 100 & 0.0020 .005 & $\begin{array}{lll}-0.001 & 0.012\end{array}$ & -0.0030 .073 & -0.0440 .084 & -0.0030 .013 & 0.0190 .042 \\
\hline & 100 & 100 & -0.0010 .006 & 0.0030 .008 & -0.0010 .030 & -0.0190 .042 & -0.0080 .016 & 0.0170 .031 \\
\hline & 250 & 250 & $0.000_{0.001}$ & 0.0010 .003 & 0.0040 .015 & -0.0080 .030 & -0.0010 .008 & -0.0030 .008 \\
\hline \multirow[t]{4}{*}{ C.3.c. } & 100 & 50 & 0.0030 .006 & $-0.000 \quad 0.013$ & 0.0200 .093 & $\begin{array}{ll}-0.041 & 0.209\end{array}$ & -0.0060 .029 & 0.0170 .046 \\
\hline & 50 & 100 & 0.0020 .005 & -0.0010 .013 & 0.0040 .148 & -0.0320 .114 & -0.0030 .017 & 0.0210 .043 \\
\hline & 100 & 100 & -0.0010 .006 & 0.0030 .008 & 0.0030 .056 & 0.0110 .064 & -0.0090 .017 & $0.018 \quad 0.031$ \\
\hline & 250 & 250 & $0.000_{0.001}$ & 0.0010 .003 & $0.009_{0.032}$ & 0.0130 .061 & -0.0020 .007 & -0.0030 .008 \\
\hline \multirow[t]{4}{*}{ C.3.d. } & 100 & 50 & 0.0030 .006 & -0.0000 .013 & $0.017_{0.095}$ & -0.0890 .154 & -0.0020 .030 & 0.0170 .046 \\
\hline & 50 & 100 & 0.0020 .005 & -0.0010 .012 & -0.0060 .140 & -0.0450 .083 & -0.0030 .013 & 0.0200 .043 \\
\hline & 100 & 100 & -0.0010 .006 & 0.0020 .008 & 0.0020 .055 & -0.0250 .045 & $-0.008 \quad 0.017$ & $0.017_{0.031}$ \\
\hline & 250 & 250 & $0.000 \quad 0.001$ & 0.0010 .003 & $0.008 \quad 0.024$ & -0.0090 .031 & -0.0010 .008 & -0.0030 .008 \\
\hline \multirow[t]{4}{*}{ C.3.e. } & 100 & 50 & 0.0040 .006 & -0.0000 .013 & $0.069 \quad 0.285$ & $-0.044 \quad 0.207$ & -0.0060 .029 & 0.0170 .046 \\
\hline & 50 & 100 & 0.0020 .004 & -0.0010 .013 & $0.008 \quad 0.431$ & -0.0330 .117 & -0.0030 .017 & 0.0210 .043 \\
\hline & 100 & 100 & -0.2000 .000 & -1.0000 .000 & -3.0000 .000 & -0.3000 .000 & -0.2000 .000 & -3.0000 .000 \\
\hline & 250 & 250 & -0.2000 .000 & -1.0000 .000 & -3.0000 .000 & -0.3000 .000 & -0.2000 .000 & -3.0000 .000 \\
\hline \multirow[t]{4}{*}{ C.3.f. } & 100 & 50 & -0.2000 .000 & -1.0000 .000 & -3.0000 .000 & -0.9000 .000 & -0.2000 .000 & -3.0000 .000 \\
\hline & 50 & 100 & -0.2000 .000 & -1.0000 .000 & -3.0000 .000 & -0.9000 .000 & -0.2000 .000 & -3.0000 .000 \\
\hline & 100 & 100 & -0.2000 .000 & -1.0000 .000 & -3.0000 .000 & -0.9000 .000 & -0.2000 .000 & -3.0000 .000 \\
\hline & 250 & 250 & -0.2000 .000 & -1.0000 .000 & -3.0000 .000 & -0.9000 .000 & -0.2000 .000 & -3.0000 .000 \\
\hline
\end{tabular}

Table 6: Simulation results for Student's dynamic panel data models with $\nu=3$. 


\begin{tabular}{|c|c|c|c|c|c|c|c|c|}
\hline & $N$ & $T$ & $\gamma$ & $\beta$ & $\sigma_{\mu}$ & $h$ & $\sigma_{\eta}$ & $\nu$ \\
\hline \multirow[t]{4}{*}{ C.1.a. } & 100 & 50 & 0.0020 .010 & -0.0020 .010 & -0.0140 .047 & & & $\begin{array}{lll}0.286 & 0.347\end{array}$ \\
\hline & 50 & 100 & -0.0040 .008 & -0.0010 .014 & $0.004_{0.045}$ & & & $0.135 \quad 0.274$ \\
\hline & 100 & 100 & -0.0010 .005 & -0.0030 .010 & $-0.010 \quad 0.036$ & & & $0.140 \quad 0.159$ \\
\hline & 250 & 250 & $0.000 \quad 0.003$ & -0.0000 .003 & 0.0020 .022 & & & $0.028 \quad 0.067$ \\
\hline \multirow[t]{4}{*}{ C.1.b. } & 100 & 50 & -0.0010 .010 & -0.0010 .010 & -0.0070 .105 & & & $0.286 \quad 0.348$ \\
\hline & 50 & 100 & -0.0040 .008 & -0.0010 .014 & $0.008_{0.091}$ & & & $0.136 \quad 0.275$ \\
\hline & 100 & 100 & -0.0010 .005 & -0.0030 .010 & -0.0120 .067 & & & $0.141 \quad 0.159$ \\
\hline & 250 & 250 & $0.000_{0.003}$ & -0.0000 .003 & $0.007_{0.044}$ & & & $0.026 \quad 0.067$ \\
\hline \multirow[t]{4}{*}{ C.1.c. } & 100 & 50 & -0.0010 .009 & -0.0010 .010 & -0.0080 .321 & & & $0.284 \quad 0.349$ \\
\hline & 50 & 100 & -0.0040 .008 & -0.0010 .014 & 0.0210 .279 & & & $0.138 \quad 0.274$ \\
\hline & 100 & 100 & -0.0010 .004 & -0.0030 .010 & -0.0200 .195 & & & $140 \quad 0.159$ \\
\hline & 250 & 250 & $0.000 \quad 0.003$ & -0.0000 .003 & $0.019_{0.131}$ & & & 0.0290 .067 \\
\hline \multirow[t]{4}{*}{ C.2.a. } & 100 & 50 & -0.0030 .008 & -0.0010 .013 & & -0.0250 .157 & $0.004_{0.021}$ & $0.059 \quad 0.234$ \\
\hline & 50 & 100 & 0.0020 .009 & -0.0030 .012 & & -0.0310 .100 & 0.0010 .024 & 0.2090 .295 \\
\hline & 100 & 100 & -0.0010 .005 & -0.0030 .009 & & 0.0950 .061 & -0.0040 .024 & $0.070 \quad 0.134$ \\
\hline & 250 & 250 & $0.000 \quad 0.003$ & 0.0000 .003 & & -0.0060 .075 & -0.0020 .010 & 0.0130 .063 \\
\hline \multirow[t]{4}{*}{ C.2.b. } & 100 & 50 & -0.0040 .009 & 0.0020 .014 & & 270.058 & $04_{0.025}$ & $0.068 \quad 0.232$ \\
\hline & 50 & 100 & 0.0020 .008 & -0.0020 .012 & & -0.0280 .052 & 0.0030 .027 & $0.205 \quad 0.295$ \\
\hline & 100 & 100 & -0.0010 .005 & 30.009 & & $0.015 \quad 0.037$ & & 90.133 \\
\hline & 250 & 250 & $0.000 \quad 0.003$ & 0.0000 .003 & & $0.000 \quad 0.021$ & -0.0030 .010 & 0.0120 .065 \\
\hline \multirow[t]{4}{*}{ C.3.a. } & 100 & 50 & -0.0050 .008 & 0.0020 .007 & -0.0090 .037 & -0.0690 .178 & $0.003_{0.027}$ & 0.0830 .306 \\
\hline & 50 & 100 & -0.0010 .011 & 0.0030 .007 & $0.000 \quad 0.056$ & -0.0730 .175 & -0.0080 .017 & 0.0790 .282 \\
\hline & 100 & 100 & -0.0010 .005 & -0.0000 .009 & -0.0040 .034 & -0.0440 .069 & 0.0090 .019 & 300.138 \\
\hline & 250 & 250 & -0.0000 .002 & -0.0000 .005 & $0.006 \quad 0.017$ & -0.0030 .046 & & 0.0150 .062 \\
\hline \multirow[t]{4}{*}{ C.3.b. } & 100 & 50 & -0.0060 .008 & 0.0010 .008 & -0.0080 .037 & -0.0360 .076 & 0.0080 .025 & 0.1120 .299 \\
\hline & 50 & 100 & -0.0010 .010 & 0.0030 .007 & -0.0040 .056 & -0.0030 .051 & -0.0050 .019 & $0.080 \quad 0.283$ \\
\hline & 100 & 100 & -0.0010 .005 & -0.0000 .010 & -0.0060 .034 & -0.0410 .076 & $0.011_{0.022}$ & $\begin{array}{ll}0.081 & 0.138\end{array}$ \\
\hline & 250 & 250 & -0.0000 .002 & -0.0000 .005 & $0.005 \quad 0.017$ & $\begin{array}{ll}-0.005 & 0.028\end{array}$ & -0.0010 .011 & $0.015 \quad 0.063$ \\
\hline \multirow[t]{4}{*}{ C.3.c. } & 100 & 50 & -0.0060 .008 & 0.0010 .008 & -0.0110 .066 & -0.0640 .194 & 0.0040 .027 & $0.115 \quad 0.304$ \\
\hline & 50 & 100 & -0.0010 .011 & 0.0030 .007 & 0.0030 .115 & $\begin{array}{lll}-0.076 & 0.172\end{array}$ & -0.0080 .017 & $\begin{array}{lll}0.081 & 0.283\end{array}$ \\
\hline & 100 & 100 & -0.0010 .005 & -0.0000 .009 & -0.0030 .064 & -0.0480 .066 & 80.019 & 0.0820 .138 \\
\hline & 250 & 250 & -0.0000 .002 & -0.0000 .005 & $0.012_{0.036}$ & -0.0040 .046 & -0.0020 .010 & \\
\hline \multirow[t]{4}{*}{ C.3.d. } & 100 & 50 & -0.0060 .008 & 0.0010 .008 & -0.0160 .067 & -0.0340 .076 & $0.008_{0.025}$ & 0.1140 .300 \\
\hline & 50 & 100 & -0.0010 .010 & 0.0020 .007 & -0.0050 .115 & -0.0080 .065 & -0.0050 .019 & 0.0820 .285 \\
\hline & 100 & 100 & -0.0010 .005 & -0.0000 .010 & -0.0070 .063 & -0.0490 .075 & $0.010_{0.022}$ & 0.0820 .138 \\
\hline & 250 & 250 & -0.0000 .002 & -0.0000 .005 & $0.011_{0.035}$ & $\begin{array}{lll}-0.006 & 0.028\end{array}$ & -0.0010 .011 & $\begin{array}{lll}0.015 & 0.063\end{array}$ \\
\hline \multirow[t]{4}{*}{ C.3.e. } & 100 & 50 & -0.0060 .008 & 0.0010 .008 & -0.0320 .188 & -0.0720 .189 & $0.004_{0.027}$ & $0.114_{0.305}$ \\
\hline & 50 & 100 & -0.0010 .010 & 0.0030 .007 & $0.011_{0.358}$ & -0.0780. & -0.0080 .017 & 0.0830 .286 \\
\hline & 100 & 100 & -0.0020 .005 & -0.0000 .009 & $0.000 \quad 0.182$ & -0.0480 .066 & 0.0080 .019 & 0.0830 .140 \\
\hline & 250 & 250 & -0.0000 .002 & -0.0000 .005 & $0.034_{0.108}$ & -0.0050 .045 & -0.0020 .010 & $0.017 \quad 0.064$ \\
\hline \multirow[t]{4}{*}{ C.3.f. } & 100 & 50 & -0.0060 .007 & 0.0010 .008 & -0.0450 .189 & -0.0440 .081 & 0.0080 .025 & 0.1130 .301 \\
\hline & 50 & 100 & -0.0010 .010 & 0.0020 .007 & -0.0040 .361 & $\begin{array}{ll}-0.0120 .067\end{array}$ & -0.0050 .019 & $0.084 \quad 0.286$ \\
\hline & 100 & 100 & -0.0020 .005 & -0.0000 .010 & -0.0060 .180 & -0.0600 .075 & $0.010_{0.021}$ & 0.0820 .139 \\
\hline & 250 & 250 & -0.0000 .002 & -0.0000 .005 & 0.0310 .106 & -0.0090 .027 & -0.0010 .011 & $\begin{array}{lll}0.015 & 0.064\end{array}$ \\
\hline
\end{tabular}

Table 7: Simulation results for Student's dynamic panel data models with $\nu=5$. 


\begin{tabular}{|c|c|c|c|c|c|c|c|c|}
\hline & $N$ & $T$ & $\gamma$ & $\beta$ & $\sigma_{\mu}$ & $h$ & $\sigma_{\eta}$ & $\nu$ \\
\hline \multirow[t]{4}{*}{ C.1.a. } & 100 & 50 & -0.0050 .011 & 0.0010 .015 & $-0.016 \quad 0.042$ & & & 531.085 \\
\hline & 50 & 100 & -0.0050 .009 & $0.006_{0.012}$ & $0.000_{0.043}$ & & & $0.130 \quad 0.982$ \\
\hline & 100 & 100 & -0.0010 .006 & -0.0040 .008 & -0.0110 .034 & & & $1.025 \quad 0.527$ \\
\hline & 250 & 250 & $0.000 \quad 0.003$ & 0.0010 .004 & $0.006 \quad 0.024$ & & & $0.241 \quad 0.295$ \\
\hline \multirow[t]{4}{*}{ C.1.b. } & 100 & 50 & -0.0060 .011 & $0.001_{0.015}$ & -0.0130 .078 & & & $1.145_{1.048}$ \\
\hline & 50 & 100 & -0.0040 .009 & $0.006 \quad 0.012$ & -0.0080 .073 & & & 0.1410 .992 \\
\hline & 100 & 100 & -0.0010 .006 & -0.0040 .008 & -0.0250 .068 & & & $1.036 \quad 0.524$ \\
\hline & 250 & 250 & $0.000_{0.003}$ & $0.001_{0.004}$ & $0.011_{0.048}$ & & & $0.242 \quad 0.296$ \\
\hline \multirow[t]{4}{*}{ C.1.c. } & 100 & 50 & -0.0050 .011 & $0.001_{0.015}$ & -0.0310 .221 & & & $1.177_{1.057}$ \\
\hline & 50 & 100 & -0.0040 .009 & $0.006 \quad 0.012$ & -0.0450 .196 & & & 0.1450 .999 \\
\hline & 100 & 100 & $0.000 \quad 0.006$ & -0.0040 .008 & -0.0850 .209 & & & 1.0410 .523 \\
\hline & 250 & 250 & $0.000 \quad 0.003$ & 0.0010 .004 & 0.0320 .142 & & & $0.243 \quad 0.297$ \\
\hline \multirow[t]{4}{*}{ C.2.a. } & 100 & 50 & -0.0010 .008 & $0.007_{0.012}$ & & -0.0390 .194 & -0.0090 .021 & -0.1640 .806 \\
\hline & 50 & 100 & -0.0060 .011 & 0.0010 .015 & & $48 \quad 0.101$ & -0.0020 .021 & 0.6840 .959 \\
\hline & 100 & 100 & -0.0020 .006 & -0.0020 .009 & & 0090.101 & -0.0040 .021 & $0.560 \quad 0.744$ \\
\hline & 250 & 250 & $0.000 \quad 0.003$ & $0.000 \quad 0.004$ & & 0210.057 & 0.0010 .009 & 0.1370 .306 \\
\hline \multirow[t]{4}{*}{ C.2.b. } & 100 & 50 & -0.0020 .009 & 0.0090 .012 & & $\begin{array}{ll}-0.035 & 0.087\end{array}$ & -0.0070 .024 & 950.841 \\
\hline & 50 & 100 & $-0.006 \quad 0.011$ & $0.001_{0.014}$ & & 0.0000 .026 & -0.0010 .021 & 580.963 \\
\hline & 100 & 100 & -0.0020 .006 & -0.0020 .009 & & -0.0090 .047 & -0.0030 .022 & $75 \quad 0.744$ \\
\hline & 250 & 250 & $0.000 \quad 0.003$ & $0.000 \quad 0.004$ & & $14_{0.033}$ & -0.0010 .009 & 360.303 \\
\hline \multirow[t]{4}{*}{ C.3.a. } & 100 & 50 & -0.0070 .013 & $0.001_{0.018}$ & 130.040 & 0.0250 .112 & $0.010_{0.019}$ & $0.290 \quad 1.058$ \\
\hline & 50 & 100 & -0.0080 .012 & 0.0010 .018 & $0.011_{0.055}$ & -0.0390 .074 & 0.0090 .021 & $0.210 \quad 1.036$ \\
\hline & 100 & 100 & -0.0000 .005 & 0.0050 .006 & -0.0060 .038 & -0.0410 .155 & -0.0050 .015 & $0.700 \quad 0.609$ \\
\hline & 250 & 250 & $0.000 \quad 0.003$ & -0.0000 .002 & 0.0010 .023 & 0.0070 .075 & -0.0000 .011 & $0.132 \quad 0.297$ \\
\hline \multirow[t]{4}{*}{ C.3.b. } & 100 & 50 & -0.0080 .013 & $0.000_{0.018}$ & 0.0190 .038 & -0.0240 .059 & $0.006 \quad 0.029$ & $0.194 \quad 1.069$ \\
\hline & 50 & 100 & -0.0080 .012 & 0.0010 .018 & $0.003 \quad 0.057$ & -0.0350 .058 & 0.0090 .025 & $0.214 \quad 1.040$ \\
\hline & 100 & 100 & -0.0000 .004 & 0.0050 .006 & -0.0090 .037 & -0.0150 .030 & -0.0030 .009 & 0.6860 .600 \\
\hline & 250 & 250 & $0.000_{0.003}$ & -0.0000 .002 & $0.000_{0.024}$ & -0.0150 .033 & $0.001_{0.011}$ & $0.132 \quad 0.298$ \\
\hline \multirow[t]{4}{*}{ C.3.c. } & 100 & 50 & -0.0080 .013 & 0.0010 .018 & 0.0420 .072 & -0.0180 .095 & 0.0080 .021 & $0.217_{1.079}$ \\
\hline & 50 & 100 & -0.0080 .012 & 0.0010 .018 & 0.0210 .102 & -0.0440 .076 & $0.009_{0.021}$ & $0.211_{1.028}$ \\
\hline & 100 & 100 & $0.000 \quad 0.005$ & 0.0050 .006 & -0.0140 .075 & -0.0450 .152 & -0.0060 .015 & $0.706 \quad 0.608$ \\
\hline & 250 & 250 & $0.000 \quad 0.003$ & -0.0000 .002 & 0.0030 .043 & $0.004_{0.075}$ & -0.0000 .010 & $0.133 \quad 0.298$ \\
\hline \multirow[t]{4}{*}{ C.3.d. } & 100 & 50 & -0.0070 .013 & 0.0000 .018 & 0.0350 .072 & -0.0250 .062 & $0.006 \quad 0.029$ & $0.215_{1.073}$ \\
\hline & 50 & 100 & -0.0080 .012 & 0.0010 .018 & $0.010_{0.109}$ & -0.0360 .058 & $0.009 \quad 0.025$ & $0.216_{1.033}$ \\
\hline & 100 & 100 & $0.000 \quad 0.004$ & 0.0050 .006 & -0.0190 .075 & -0.0170 .030 & -0.0030 .009 & 0.7010 .589 \\
\hline & 250 & 250 & $0.000 \quad 0.003$ & -0.0000 .002 & $0.000 \quad 0.046$ & -0.0140 .033 & 0.0010 .011 & $0.133 \quad 0.299$ \\
\hline \multirow[t]{4}{*}{ C.3.e. } & 100 & 50 & -0.0060 .012 & 0.0010 .018 & $0.117_{0.213}$ & -0.0260 .093 & 0.0070 .021 & $0.229 \quad 1.076$ \\
\hline & 50 & 100 & -0.0080 .011 & 0.0010 .018 & $0.056 \quad 0.296$ & -0.0450 .076 & $0.008_{0.021}$ & $0.210_{1.023}$ \\
\hline & 100 & 100 & 0.0010 .005 & 0.0050 .006 & -0.0510 .227 & -0.0480 .151 & -0.0060 .015 & $0.709 \quad 0.607$ \\
\hline & 250 & 250 & $0.000_{0.003}$ & -0.0000 .002 & $0.011_{0.126}$ & 0.0010 .076 & -0.0010 .010 & $0.133 \quad 0.299$ \\
\hline \multirow[t]{4}{*}{ C.3.f. } & 100 & 50 & -0.0060 .011 & $0.000 \quad 0.018$ & $0.107_{0.207}$ & -0.0520 .094 & 0.0050 .029 & $0.224 \quad 1.070$ \\
\hline & 50 & 100 & -0.0080 .011 & 0.0010 .018 & 0.0570 .300 & -0.0550 .053 & $0.010_{0.025}$ & $0.210_{1.02}$ \\
\hline & 100 & 100 & $0.001_{0.004}$ & 0.0050 .006 & -0.0520 .229 & -0.0330 .028 & -0.0030 .009 & $0.705 \quad 0.586$ \\
\hline & 250 & 250 & $0.000 \quad 0.003$ & -0.0000 .002 & 0.0020 .129 & -0.0170 .034 & 0.0010 .011 & $0.127_{0.297}$ \\
\hline
\end{tabular}

Table 8: Simulation results for Student's dynamic panel data models with $\nu=10$. 

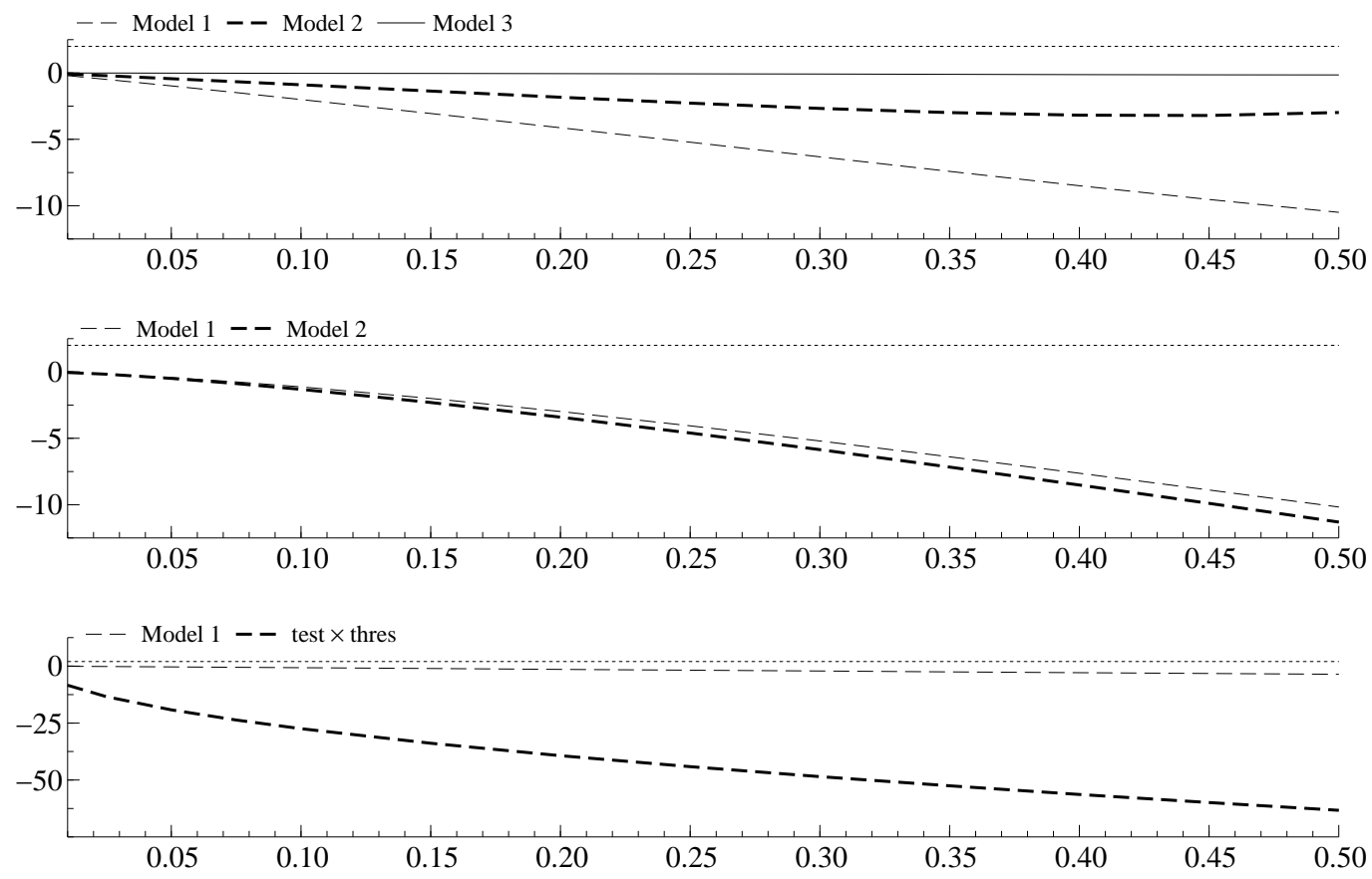

Figure 10: Diagnostic checks for the empirical studies. The t-test statistics correspond to the estimated models presented for: (i) the union choice of male, (ii) the crime rates of families and (iii) economic growth rates. All tests are implemented as discussed in Section 2.1. 


\section{References}

Anderson, B. D. O. \& Moore, J. B. (1979), Optimal Filtering, Prentice-Hall, Englewood Cliffs.

Baltagi, B. H. (2005), Econometric Analysis of Panel Data, John Wiley \& Sons, New York.

Durbin, J. \& Koopman, S. J. (1997), 'Monte Carlo maximum likelihood estimation of nonGaussian state space models', Biometrika 84, 669-684.

Durbin, J. \& Koopman, S. J. (2000), 'Time series analysis of non-Gaussian observations based on state space models from both classical and Bayesian perspectives', Journal of the Royal Statistical Society, Series B 62, 3-56.

Durbin, J. \& Koopman, S. J. (2002), 'A simple and efficient simulation smoother for state space time series analysis', Biometrika 89, 603-616.

Durbin, J. \& Koopman, S. J. (2012), Time Series Analysis by State Space Methods, 2 edn, Oxford University Press, Oxford.

Jungbacker, B. \& Koopman, S. J. (2007), 'Monte Carlo Estimation for Nonlinear NonGaussian State Space Models', Biometrika 94, 827-839.

Jungbacker, B. \& Koopman, S. J. (2008), 'Likelihood-based Analysis for Dynamic Factor Models'. Tinbergen Institute Discussion Paper: 08-007/4.

Koopman, S. J. \& Durbin, J. (2003), 'Filtering and smoothing of of state vector for diffuse state space models', Journal of Time Series Analysis 24, 85-98.

Mesters, G. \& Koopman, S. J. (2014), 'Generalized Dynamic Panel Data Models with Random Effects for Cross-Section and Time'. Tinbergen Institute Working paper TI 2012$009 / 4$.

Nocedal, J. \& Wright, S. J. (1999), Numerical Optimization, Springer Verlag, New York.

Roy, S. N. (1958), 'A Note On Inverting a Certain Type of Patterned Matrix'. United States. Air Force Office of Scientific Research Technical Notes No. 191.

Roy, S. N. \& Sarhan, A. E. (1956), 'On Inverting a Class of Patterned Matrices', Biometrika 43, 227-231.

Shephard, N. \& Pitt, M. K. (1997), 'Likelihood analysis of non-Gaussian measurement time series', Biometrika 84, 653-667.

So, M. K. P. (2003), 'Posterior mode estimation for nonlinear and non-Gaussian state space models', Statistica Sinica 13, 255-274. 